\title{
NONUNIQUENESS AND HIGH ENERGY SOLUTIONS FOR A CONFORMALLY INVARIANT SCALAR EQUATION
}

\author{
DANIEL POLLACK
}

\section{INTRODUCTION}

The Yamabe problem asserts that given any compact Riemannian manifold $(M, \bar{g})$, without boundary and of dimension greater than or equal to three, there exists a conformally related metric $g$, with constant scalar curvature. A glimpse into the colorful history of this problem is given by $[20,19,1,14]$. Following Schoen's resolution of the Yamabe problem [14], there has been great effort and success in better understanding the quantity and behavior of metrics which can occur as solutions. In this paper we prove the existence of arbitrarily many distinct solutions to the Yamabe problem with constant positive scalar curvature, all lying within a fixed conformal class which is arbitrarily close, in the $C^{0}$ topology, to the conformal class of any given metric of positive scalar curvature. Before stating the result precisely we recall some of what is know about the existence of metrics of constant scalar curvature. For a complete and accessible discussion of the Yamabe problem we refer the reader to the excellent survey article by J. Lee and T. Parker [9].

Constant scalar curvature metrics arise as the critical points of the total scalar curvature functional

$$
\mathcal{R}(g)=\int_{M} R(g) d v_{g}
$$

restricted to those $g \in[\bar{g}]_{1}$, the space of metrics which are conformally equivalent to $\bar{g}$ and have unit volume. If we write $g=u^{\frac{4}{n-2}} \bar{g}$, for some $u \in C^{\infty}(M)$, $u>0$, then the scalar curvature $R(g)$ is given by

$$
R(g)=-c(n)^{-1} u^{-\frac{n+2}{n-2}} L_{\bar{g}} u
$$


where $c(n)=\frac{n-2}{4(n-1)}$, and $L_{\bar{g}}$ is the linear operator given by

$$
L_{\bar{g}}=\Delta_{\bar{g}}-c(n) R(\bar{g}) .
$$

$L_{\bar{g}}$ is called the conformal Laplacian of $(M, \bar{g})$, it is a conformally invariant operator in the sense that if $g=u^{\frac{4}{n-2}} \bar{g}$, then for any function $\phi \in C^{\infty}(M)$ we have $L_{\bar{g}}(u \phi)=u^{\frac{n+2}{n-2}} L_{g} \phi$. The Euler-Lagrange equation of $(0.1)$ is $R(g) \equiv K$, for some constant $K$, or equivalently

$$
L_{\bar{g}} u+c(n) K u^{\frac{n+2}{n-2}}=0 .
$$

This is known as Yamabe's equation and will be the focus of our study.

The approach used in solving the Yamabe problem was to seek a metric $g$ which minimizes (0.1). The Yamabe Invariant is defined by

$$
I(\bar{g})=\inf \left\{\mathcal{R}(g): g \in[\bar{g}]_{1}\right\} .
$$

Thus the solution of the Yamabe problem produces a metric $g \in[\bar{g}]_{1}$ with constant scalar curvature $R(g)=\mathcal{R}(g)=I(\bar{g})$. Clearly by the definition, $I(\bar{g})$ is a conformal invariant. We thus refer to the sign of a conformal class, $[\bar{g}]$ as being the sign of $I(\bar{g})$. The sign of $I(\bar{g})$ dictates, to a large degree, the behavior of $(0.3)$ and the types of solutions which can arise. $I(\bar{g})>0$, (respectively $=0$, $<0)$ is equivalent to the existence of a metric $g \in[\bar{g}]$ with scalar curvature $R(g)>0$, (respectively $=0,<0)$.

For nonpositive conformal classes, the Yamabe problem has a unique solution among unit volume metrics. We deal exclusively with positive conformal classes. Among positive conformal classes, uniqueness does hold under very special circumstances. Recall that an Einstein metric $g$ is a metric whose Ricci curvature satisfies $\operatorname{Ric}(g)-\frac{R(g)}{n} g=0$. Einstein metrics arise as critical points of the total scalar curvature functional (0.1) taken over the space of all unit volume metrics on M. A theorem of M. Obata [11], asserts that in the conformal class of an Einstein metric, there exists a unique metric of constant scalar curvature and unit volume, namely the Einstein metric suitably normalized. This holds provided that the conformal class of the Einstein metric is not the conformal class of the round metric $g_{0}$, on $S^{n}$. The proviso that one avoid the conformal class of $g_{0}$, is necessary since there exists an $n+1$ dimensional family of metrics in $\left[g_{0}\right]_{1}$ with constant scalar curvature. These metrics arise as the 
pullback of $g_{0}$ by the nonisometric, conformal diffeomorphisms of $S^{n}$, the space of which may be identified with the interior of the unit ball $\mathbb{B}^{n+1} \subset \mathbb{R}^{n+1}$. This exceptional property of $\left(S^{n}, g_{0}\right)$ is heavily exploited in this paper, to show that the uniqueness property exhibited by Einstein metrics which are conformally distinct from $g_{0}$, is not a general phenomenon.

Recently R. Schoen has completed a thorough analysis of solutions to (0.3) which shows that if $(M, \bar{g})$ is not conformally equivalent to $\left(S^{n}, g_{0}\right)$, then the set of solutions form a compact family, the size of which is determined solely by the conformal class, $[\bar{g}]$. In $[16,17,18]$ Schoen considers the family of equations, indexed by the exponent $p \in\left[1, \frac{n+2}{n-2}\right]$, given by

$$
L_{\bar{g}} u+E(u) u^{p}=0
$$

where $E(u)$ is a positive constant which depends on the solution. When $p<$ $\frac{n+2}{n-2}$, these equations are referred to as the subcritical regularization of $(0.3)$. This approach to the study of (0.3) was originally introduced by Yamabe. For exponents $p<\frac{n+2}{n-2}$ the Sobolev embedding $i_{p}: H^{1}(M) \hookrightarrow L^{p+1}(M)$, is actually compact by the Rellich-Kondrakov theorem. This accounts for the fact that the subcritical equations, (0.5) with $p<\frac{n+2}{n-2}$, are more easily analyzed then (0.3) which has the critical exponent $p=\frac{n+2}{n-2}$ for which the embedding is still continuous but fails to be compact. This loss of compactness is the source of much of the difficulty of the Yamabe Problem. By a careful analysis of the possible types of blow up which could occur as $p \rightarrow \frac{n+2}{n-2}$, Schoen has derived uniform estimates on $(0.5)$ provided that $(M, \bar{g})$ is not conformally equivalent to $\left(S^{n}, g_{0}\right)$. These estimates imply that there exists a constant $\Lambda=\Lambda(\bar{g})$, which depends only on the conformal class of $\bar{g}$, such that every solution of $(0.5)$ for any $p \in\left[1, \frac{n+2}{n-1}\right]$, lies in the set $\Omega_{\Lambda}$ defined by $\Omega_{\Lambda}=\left\{u \in C^{2, \alpha}(M):\|u\|_{2, \alpha}<\Lambda\right\}$. This shows that no blow up can occur, and all solutions of (0.5) converge as $p \rightarrow \frac{n+2}{n-2}$, to a solution of Yamabe's equation $(0.3)$. In the case when all the solutions to $(0.3)$ in $[\bar{g}]_{1}$ are nondegenerate, as will be true for a generic conformal class, the existence theory which follows from this estimate implies that there are at most a finite number of solutions within $[\bar{g}]$.

These results provide some motivation for why we need to to perturb the 
given conformal class in order to find arbitrarily many solutions with arbitrarily high energy. In particular, they explain why these solutions do not exist without a perturbation of the conformal class.

The energy of a solution is given by

$$
E(u)=\int_{M}\left(|\nabla u|^{2}+c(n) R(\bar{g}) u^{2}\right) d v_{\bar{g}} .
$$

In the geometric case of the critical exponent $p=\frac{n+2}{n-2}$, for $g=u^{\frac{4}{n-2}} \bar{g}$ we have $\mathcal{R}(g)=c(n)^{-1} E(u)$. If we normalize our solutions so that $R(g)=n(n-1)$, thus leaving the volume uncontrolled, the energy of our solutions is then dependent on the volume of $(M, g)$

$$
E(u)=\frac{n(n-2)}{4} \operatorname{Vol}_{g}(M) .
$$

The solution to Yamabe's problem produced a global minimum for the variational problem (0.1). This minimum always has energy less than or equal to that of the unit sphere $S^{n} \subset \mathbb{R}^{n+1}$ with the induced metric $g_{0}$, and equality occurs if and only if $(M, g)$ is conformally equivalent to $\left(S^{n}, g_{0}\right)$. Thus, if we let $\omega_{n}$ denote the volume of the unit sphere, then for a minimizing solution $g_{\min }=u_{\min }^{\frac{4}{n-2}} \bar{g}$ we have

$$
E\left(u_{\min }\right) \leq \frac{n(n-2)}{4} \omega_{n}
$$

We note that the Morse index of the solution $g=u^{\frac{4}{n-2}} \bar{g}$ is equal to the number of eigenvalues of $\Delta_{g}$ in $(0, n)$. Thus if we assume that $g$ is a minimal solution then we know that $\lambda_{1}$, the first nonzero eigenvalue for $\Delta_{g}$ on $M$, satisfies $\lambda_{1} \geq n$. The futher assumption that the solution is nondegenerate insures that the strict inequality $\lambda_{1}>n$ holds.

We are now in a position to give a precise statement of our result.

Theorem 0.1. Let $(M, g)$ be a compact Riemannian manifold without boundary and of dimension $n \geq 3$, with positive scalar curvature, $R(g)>0$. Given any integer $N \geq 1$ and any number $\bar{\epsilon}>0$, there exists a conformal class $\left[g_{1}\right]$ which satisfies

$$
\left\|g^{\prime}-g_{1}{ }^{\prime}\right\|_{C^{\circ}}<\bar{\epsilon}
$$


for some metric $g^{\prime} \in[g]$ and $g_{1}{ }^{\prime} \in\left[g_{1}\right]$, and which contains metrics $g_{1}, \ldots, g_{N}$ such that for each $k=1, \ldots, N$

$$
g_{k}=u_{k}^{\frac{4}{n-2}} g_{1} \quad \text { and } \quad R\left(g_{k}\right) \equiv n(n-1) .
$$

Moreover the energy $E\left(u_{k}\right)$, of each of these solutions can be estimated by

$$
\left|E\left(u_{k}\right)-\left(\operatorname{Vol}_{g}(M)+(k+1) \omega_{n}\right)\right| \leq c\left(\bar{\epsilon}^{\frac{n-2}{2}}+\bar{\epsilon}^{\frac{n-2}{q}}\right),
$$

for any $q>\frac{n}{2}$ and a constant $c=c(n, g)$.

Thus there exists a sequence of metrics $g_{\bar{\epsilon}}$, each with at least $N$ distinct solutions in their conformal classes, which converge to $g$ in the $C^{0}$ topology, as $\bar{\epsilon} \rightarrow 0$. This is valid even though $g$ may only have one solution in its conformal class, e.g. if $g$ is Einstein, or if all solutions in $g$ are minimizing, as is the case for $g=g_{0}$ on $S^{n}$. Note that the energy estimate in Theorem 0.1 implies that each of the solutions $g_{k}$ are nonminimizing. If we let

$$
\mathcal{M}_{N}=\{[g]: \exists \geq N \text { solutions of Yamabe's equation in }[g]\}
$$

then we have the following Corollary.

Corollary 0.2. For any integer $N \geq 1$ the set $\mathcal{M}_{N}$ is dense in the $C^{0}$ norm on the space of positive conformal classes.

One should note that Schoen's estimates, referred to above, will be stable in a sufficiently strong topology. In other words, the same set $\Omega_{\Lambda}$ should contain all the solutions to $(0.5)$ with respect to any metric $\tilde{g}$, which is $\epsilon$ close to $\bar{g}$ in the $C^{k}$ norm, for $k$ sufficiently large. Thus one can view the two results as being in opposition to each other.

In section 2 we construct approximate solutions to (0.3), which take the form of $M$ joined with a string of $k+1$ spheres, for $k=1, \ldots, N$, each attached to the next by thin necks. The resulting metrics $g_{1_{k}}$ are approximate solutions in the sense that their scalar curvature is bounded and the set where the scalar curvature is not constant, has small volume with respect to the metric $g_{1_{k}}$. It is possible to construct $N$ distinct approximate solutions $g_{1_{1}}, \ldots, g_{1_{N}}$ within a fixed conformal class, each of the form of $M$ joined to a string of $2, \ldots, N+1$ spheres respectively. 
If we treat $g_{1_{k}}$ as our new background metric and seek $g=u^{\frac{4}{n-2}} g_{1_{k}}$ with $R(g)=n(n-1)$ then, $(0.3)$ becomes

$$
\Delta_{g_{1_{k}}} u-c(n) R\left(g_{1_{k}}\right) u+\frac{n(n-2)}{4} u^{\frac{n+2}{n-2}}=0 .
$$

The statement that $g_{1_{k}}$ is an approximate solution, means that $u=1$ is an approximate solution to (0.6). Since we wish to find a solution to (0.6) which is close to our approximate solution $u=1$, we write $g=(1+\eta)^{\frac{4}{n-2}} g_{1_{k}}$ where $\eta$ is small. By linearizing (0.6) about $u=1$, our equation takes the final form

$$
\Delta_{g_{1_{k}}} \eta+n \eta=c(n)\left(R\left(g_{1_{k}}\right)-n(n-1)\right)(1+\eta)+Q(\eta)
$$

where for $\eta$ small, $Q(\eta)$ is quadratically small. For simplicity, we write $(0.7)$ as

$$
\mathcal{L} \eta=F(x, \eta)
$$

The initial analytic difficulty in solving (0.7) is the possible presence of a kernel for the linear operator $\mathcal{L}$. Such a kernel could arise from the linear functions on $S^{n} \subset \mathbb{R}^{n+1}$. In section 3 we show that these functions actually do give rise to a small eigenspace $K$ which necessarily contains any functions $\eta$ in the kernel of $\mathcal{L}$ on $M$. This is done by identifying an explicit approximate kernel $K_{0}$, and showing that there is a small eigenspace $K \subset L^{2}(M)$, very close to $K_{0}$. This explicit control on $K$ allows us to identify and quantify the component of $F(x, \eta)$ lying in $K$. This is one of the central features which allow us to solve (0.7) exactly.

We then show that we can invert the operator $\mathcal{L}$ on $K^{\perp}$, and obtain precise estimates on the solution $\eta \in K^{\perp}$ of

$$
\mathcal{L} \eta=f
$$

for $f \in K^{\perp}$. In section 4 these estimates and the structure of the approximate solution, $g_{1_{k}}$, are used to solve $(0.7)$ by a contraction mapping argument, provided that $F(x, \eta) \in K^{\perp}$.

The high degree of flexibility in our construction insures that the approximate solution may be deformed in such a way as to guarantee that the corresponding $F(x, \eta)$ is orthogonal to the small eigenspace $K$ of $\mathcal{L}$. It has been 
well known for some time that one can construct approximate solution metrics. In particular, O. Kobayashi [8] has constructed approximate solutions with arbitrarily high energy within any positive conformal class. However these are constructed by an ODE method and do not have enough parameters to be deformed into exact solutions. The fact that our approximate solution construction has a large space of parameters which determine the corresponding metric $g_{1_{i}}$, is the source of the flexibility which is necessary to solve the nonlinear equation exactly. A primary component of the deformations we use, is the recognition that an integral identity having its origins in works of $\mathrm{S}$. Pohozaev [12], and J. Kazdan and F. Warner [7], provides us with a precise measurement of the component of $F(x, \eta)$ lying in $K$. (The referee and Jose F. Escobar have pointed out that his identity is related to the Rellich identity [13], which characterizes the eigenvalues for the Dirichlet problem in terms of a boundary integral involing the normal derivative.) Moreover we show that it is possible to adjust each approximate solution, according to the required deformation, so that the deformations preserve the conformal class of the approximate solution. By doing this for each of the $1, \ldots, N$ approximate solutions we find $N$ distinct exact solutions to (0.7). The energy estimate is then a consequence of our construction of the approximate solutions.

The analytical methods used in this paper originate in the work of $\mathrm{R}$. Schoen. In [15], Schoen proves the existence of weak solutions of (0.3) on $S^{n}$ which have prescribed singular behavior. In particular, he constructs metrics $g$, conformally equivalent to $g_{0}$, which have constant scalar curvature, $n(n-1)$ and are complete on $S^{n} \backslash\left\{q_{1}, \ldots, q_{k}\right\}$, where $\left\{q_{i}\right\}_{i=1}^{k}$ is any prescribed set of $k$ points, for $k \geq 2$. In the interest of avoiding undue repetition, we will we often refer to [15].

We avoid some of the analytic difficulties of [15] by the fact that our approximate solutions are compact, whereas in [15] they are noncompact and, in certain cases can have exponential volume growth. In the noncompact case, the small eigenspace $K$ of $\mathcal{L}$, is infinite dimensional and the solutions of the linearized operator do not, in general, lie in $L^{2}(M)$. On the other hand, in our case we must keep track of the conformal class of the approximate solutions and the conformal class of their deformations to insure that we can find 
our $N$ distinct solutions all within one fixed conformal class. We also need to guarantee that neither the original manifold $(M, g)$ nor the final sphere which we attach to our string, give any contribution to the kernel of $\mathcal{L}$. This is accomplished by a generic perturbation of the metrics and, in the locally conformally flat case, accounts for our $C^{0}$ perturbation of the conformal class. In the case that $(M, g)$ is nowhere conformally flat, we require an additional perturbation to make it conformally flat in the neighborhood of a point. N. Kapouleas has used similar techniques in constructing a multitude of constant mean curvature surfaces in $\mathbb{R}^{3}$ (see $[6,5]$ ). His approximate solutions are constructed from Delaunay surfaces, a classical one parameter family of constant mean curvature surfaces. These surfaces serve the same role in his construction, as the $n+1$ parameter family of constant scalar curvature metrics on $S^{n}$ serve in ours.

Acknowledgement. This work was part of the authors doctoral dissertation at Stanford University under the direction of Richard M. Schoen, our gratitude for his support during that time can not be overstated. We would also like to thank Rafe Mazzeo and Karen Uhlenbeck for many helpful and enlightening discussion concerning this work.

\section{Approximate Solutions}

1.1. The Initial Background Metric. If we assume that $R(\bar{g})=n(n-1)$, the scalar curvature of the unit sphere $S^{n} \subset \mathbb{R}^{n+1}$ and seek metrics $g \in[\bar{g}]$ satisfying $R(g)=n(n-1)$, then writing $g=u^{\frac{4}{n-2}} \bar{g}$ for some $u>0, u \in$ $C^{\infty}(M), u$ must satisfy

$$
L_{\bar{g}} u+\frac{n(n-2)}{4} u^{\frac{n-2}{n+2}}=0
$$

where $L_{\bar{g}}$ is the conformal Laplacian with respect to the backround metric $\bar{g}$,

$$
L_{\bar{g}} u=\Delta_{\bar{g}} u-c(n) R(\bar{g}) u=\Delta_{\bar{g}} u-\frac{n(n-2)}{4} u,
$$

here $c(n)=\frac{n-2}{4(n-1)}$. We will require that $\bar{g}$ lie in a nondegenerate conformal class. This is equivalent to the invertibility of the operator $\mathcal{L}$, obtained by linearizing (1.1) about any solution $u$. Among positive conformal classes of metrics, this condition is generically satisfied, i.e. it is satisfied by an open 
and dense set of conformal classes. We will employ this condition in a very explicit manner. Since $u \equiv 1$ is a solution to (1.1), and the linearization about about this solution is

$$
\mathcal{L}=\Delta_{\bar{g}}+n,
$$

the nondegeneracy of the conformal class $[\bar{g}]$ implies that $n$ is not an eigenvalue of $-\Delta_{\bar{g}}$. This fact will be of central importance in our construction of solutions to (1.1). Recall that on $\left(S^{n}, g_{0}\right), n$ is an eigenvalue of $-\Delta_{g_{0}}$, with an $n+1$ dimensional eigenspace consisting of the restrictions of the linear functions in $\mathbb{R}^{n+1}$ to $S^{n}$. Thus $\left[g_{0}\right]$ is a degenerate conformal class. Since the kernel of $\mathcal{L}$ on $S^{n}$ can be explicitly identified, it will be possible for us to use $S^{n}$ in our construction of approximate solutions. We will also need to consider nondegenerate conformal classes of metrics on $S^{n}$. Since the nondegeneracy of $[g]$, for metrics $g$ on $S^{n}$, is a generic condition, we may consider nondegenerate metrics which are arbitrarily close to $g_{0}$. We will let $\bar{g}_{0}$ denote a metric whose conformal class is positive and nondegenerate, and which satisfies for $\epsilon>0$ and for some integer $k>0,\left|\bar{g}_{0}-g_{0}\right|_{k} \leq \epsilon$, where $|\cdot|_{k}$ denotes the $C^{k}$ norm on tensors, taken with respect to the fixed metric $g_{0}$.

The first task will be to construct $N$ approximate solutions $g_{1_{1}}, \ldots, g_{1_{N}}$ to (1.1) all lying within a fixed conformal class. Toward this end it is necessary to deform $\mathrm{g}$ to make it conformally flat in the neighborhood of some point $x_{0} \in M$. We deform $g$ as follows. Let $\|W\|$ denote the norm of the Weyl tensor $W=W_{i j k l}$, taken with respect to $g$; this is a continuous function on $M$, so let $x_{0}$ be a point at which $\|W\|$ attains its minimum value. Let $(r, \theta)$ denote polar normal coordinates centered around $x_{0}=0$. In these coordinates, $\mathrm{g}$ takes the form $g=d r^{2}+r^{2} h_{r}$ where $h_{r}=h_{i j}(r, \theta) d \theta^{i} d \theta^{j}$ is a metric on $S^{n-1}$, and $h_{0}$ denotes the standard metric on $S^{n-1}$. Let $\Psi(r)$ be a smooth nondecreasing cut-off function which satisfies $\Psi(r)=0$ for $r \leq 1, \Phi(r)=1$ for $r \geq 2$ and $\left|\Psi^{\prime}(r)\right|+\left|\Psi^{\prime \prime}(r)\right| \leq c$ for some constant $c$. We then define $\Psi_{\rho}(r)=\Psi\left(\rho^{-1} r\right)$, so that $\rho\left|\Psi_{\rho}^{\prime}(r)\right|+\rho^{2}\left|\Psi_{\rho}^{\prime \prime}(r)\right| \leq c$. For any $\rho>0$ such that $2 \rho<i(M, g)=$ the injectivity radius of $(M, g)$, we deform our metric $g$ to a new metric $g_{\rho}$ defined by

$$
g_{\rho}= \begin{cases}d r^{2}+r^{2}\left(\left(1-\Psi_{\rho}(r)\right) h_{0}+\Psi_{\rho}(r) h_{r}\right) & \text { on } B_{2 \rho}\left(x_{0}\right) \\ g & \text { on } M \backslash B_{2 \rho}\left(x_{0}\right)\end{cases}
$$


Note that $g_{\rho}$ is Euclidean in $B_{\rho}\left(x_{0}\right)$, and hence conformally flat in a neighborhood of $x_{0}$. R. Schoen has observed [14] that for $\rho$ sufficiently small, the conformal class of $g_{\rho},\left[g_{\rho}\right]$ is positive. For such $\rho$, we find, by appealing to the resolution of the Yamabe problem, a new metric, again denoted by $g$, in $\left[g_{\rho}\right]$ with constant scalar curvature $n(n-1)$, and which is a minimal solution for the variational problem. This metric is conformally flat in a neighborhood of $x_{0}$, and will serve as our new background metric. Since we can also apply such a deformation to the metric $\bar{g}_{0}$ on $S^{n}$, we shall assume that $\bar{g}_{0}$ is nondegenerate, conformally flat in a neighborhood of a point $x_{0} \in S^{n}$, satisfies $R\left(\bar{g}_{0}\right) \equiv n(n-1)$, and is a minimal solution for the variational problem. Before proceeding with the construction of our approximate solutions, we need to develop some facts concerning conformally flat metrics and the standard sphere $\left(S^{n}, g_{0}\right)$.

1.2. Stereographic Coordinates and Conformally Flat Metrics. We will always regard $S^{n}$ as the unit sphere in $\mathbb{R}^{n+1}$. If $q$ is any point in $S^{n}$, let $\mathbf{P}(q) \subset \mathbb{R}^{n+1}$ denote the hyperplane passing through the origin and orthogonal to $q$, and let $x=\left(x^{1}, \ldots, x^{n}\right)$ denote Euclidean coordinates in $\mathbf{P}(q) . \mathbb{R}^{n}=$ $\mathbf{P}(q)$ can be identified with $S^{n} \backslash\{q\}$ by stereographic projection from $q$. For $p \in \mathbb{R}^{n+1}$, let $\xi(p)=p \cdot q$, we may then view $(x, \xi)=\left(x^{1}, \ldots, x^{n}, \xi\right)$ as Euclidean coordinates for $\mathbb{R}^{n+1}$. Stereographic projection $\pi: \mathbb{R}^{n} \rightarrow S^{n}$ is then given by

$$
\pi(x)=\left(\frac{2 x}{|x|^{2}+1}, \frac{|x|^{2}-1}{|x|^{2}+1}\right) .
$$

The standard metric $g_{0}$ on $S^{n} \subset \mathbb{R}^{n+1}$, is then

$$
g_{0}=\frac{4}{\left(1+|x|^{2}\right)^{2}} \sum_{i=1}^{n}\left(d x^{i}\right)^{2}
$$

when expressed in these coordinates. Let $B_{r}(q)$ denote the geodesic ball on $S^{n}$ of radius $r<\pi$ centered at $q$. A little elementary trigonometry then shows that

$$
\pi\left(\left\{x:|x|=\left(\tan \frac{1}{2} r\right)^{-1}\right\}\right)=\partial B_{r}(q)
$$

and

$$
\left|r^{-1}-\left(\tan \frac{1}{2} r\right)^{-1}\right| \leq c r
$$


The fact that $(M, g)$ is conformally flat in a neighborhood, $B_{r_{0}}\left(x_{0}\right)$ of $x_{0} \in$ $M$, is equivalent to the existence of a conformal diffeomorphism, $\Phi$ between $B_{r_{0}}\left(x_{0}\right)$ and a domain in $\left(S^{n}, g_{0}\right)$, provided $r_{0}$ is sufficiently small. If $\Phi\left(x_{0}\right)=q$, for some $q \in S^{n}$, we may write $g=\phi^{-2} g_{0}$ in $\Omega=\Phi\left(B_{r_{0}}\left(x_{0}\right)\right) \subset S^{n}$, for some $\phi \in C^{\infty}(\Omega), \phi>0$. Let $\Lambda>0$ satisfy $\phi>\Lambda^{-1}$ on $\Omega$, and $\|\phi\|_{2}<\Lambda$, where $\|\cdot\|_{2}$ denotes the $C^{2}$ norm taken with respect to the standard metric $g_{0}$ on $S^{n}$. In stereographic coordinates on $S^{n} \backslash\{q\}, g$ takes the form,

$$
g=4\left(\phi(\pi(x))+\phi(\pi(x))|x|^{2}\right)^{-2} \sum_{i=1}^{n}\left(d x^{i}\right)^{2} .
$$

This expression holds in $\mathbb{R}^{n} \backslash B_{R_{0}}(0)$, for some $R_{0}>>1$. If we let $v^{\frac{4}{n-2}}(x)=$ $\left(\phi(\pi(x))+\phi(\pi(x))|x|^{2}\right)^{-2}$, then we have,

$$
v(x)=|x|^{2-n}\left(\phi(\pi(x))+\phi(\pi(x))|x|^{-2}\right)^{\frac{2-n}{2}} .
$$

We now show that by making a fixed linear change of coordinates in $\mathbb{R}^{n}$, we can rewrite this as,

$$
\bar{v}(x)=|x|^{2-n}\left(a_{0}+h(x)\right)^{\frac{2-n}{2}},
$$

where $a_{0}=\phi(q)>0$ and $h(x)=O\left(|x|^{-2}\right)$. This type of adjustment was used by Gidas, $\mathrm{Ni}$, and Nirenberg [3] in showing that all global solutions on $\mathbb{R}^{n}$ to (1.1) are spherically symmetric.

Lemma 1.1. Given any conformally flat metric $g$ on $B_{r_{0}}\left(x_{0}\right) \subset M$, there exists an $R_{0}>>1$, and coordinates on $B_{r_{0}}\left(x_{0}\right) \backslash\left\{x_{0}\right\}$ such that

$$
g=4 \bar{v}^{\frac{4}{n-2}}(x) \sum_{i=1}^{n}\left(d x^{i}\right)^{2}
$$

for $|x|>R_{0}$, and $\bar{v}(x)$ takes the form

$$
\bar{v}(x)=|x|^{2-n}\left(a_{0}+h(x)\right)^{\frac{2-n}{2}},
$$

where $a_{0}>0$ and $h(x)=O\left(|x|^{-2}\right)$, as $|x| \rightarrow \infty$.

Proof. We first extend $\phi$ from $\Omega \subset S^{n}$ to a small neighborhood of $\Omega$ in $\mathbb{R}^{n}$ so that $\frac{\partial^{k} \phi}{\partial \xi^{k}}(q)=0, \forall k>1$. Writing the Taylor expansion for $\phi(x, \xi)$ about 
$q=(0, \ldots, 0,1) \in S^{n}$, as a function on $\mathbb{R}^{n+1}$, we have

$$
\phi(x, \xi)=a_{0}+\sum_{i=1}^{n} \phi_{i}(q) x^{i}+\frac{1}{2} \sum_{i, j=1}^{n} \phi_{i j}(q) x^{i} x^{j}+O\left(|x|^{3}\right)
$$

where $\phi_{i}=\frac{\partial \phi}{\partial x^{i}}, \phi_{i j}=\frac{\partial^{2} \phi}{\partial x^{i} \partial x^{j}}$. In stereographic coordinates this expression is transformed into an Laurent expansion about infinity, if we only keep track of those terms up to order $|x|^{-1}$, we then have,

$$
\phi(\pi(x))=a_{0}+2 \sum_{i=1}^{n} \frac{\phi_{i}(q) x^{i}}{|x|^{2}+1}+O\left(|x|^{-2}\right)
$$

Thus,

$$
v(x)^{\frac{4}{n-2}}=\frac{1}{a_{0}^{2}|x|^{4}}\left(1-\frac{2}{a_{0}} \sum_{i=1}^{n} \frac{\phi_{i}(q) x^{i}}{|x|^{2}+1}+O\left(|x|^{-2}\right)\right) .
$$

If we were to replace $x$ with $x-x_{0}$, for some $x_{0}=\left(x_{0}^{1}, \ldots, x_{0}^{n}\right) \in \mathbb{R}^{n}$, then since

$$
\frac{1}{\left|x-x_{0}\right|^{4}}=\frac{1}{|x|^{4}}\left(1+\frac{4}{|x|^{2}} \sum_{i=1}^{n} x_{0}^{i} x^{i}+O\left(|x|^{-2}\right)\right),
$$

this would have the net effect of introducing a new term of order $|x|^{-1}$ into our expansion. Explicitly for $\ell_{x_{0}}(x)=x^{\prime}=x-x_{0}$ we have,

$$
v\left(x^{\prime}\right)^{\frac{4}{n-2}}=\frac{1}{a_{0}^{2}|x|^{4}}\left(1+\frac{2}{a_{0}} \sum_{i=1}^{n} \frac{\left(2 a_{0} x_{0}^{i}-\phi_{i}(q)\right) x^{i}}{\left|x-x_{0}\right|^{2}+1}+O\left(|x|^{-2}\right)\right) .
$$

Therefore, choosing $x_{0}^{i}=\frac{\phi_{i}(q)}{2 a_{0}}$, we have

$$
\bar{v}(x)^{\frac{4}{n-2}}=\frac{1}{a_{0}^{2}|x|^{4}}\left(1+O\left(|x|^{-2}\right)\right),
$$

or simply

$$
\bar{v}(x)^{\frac{4}{n-2}}=|x|^{-4}\left(a_{o}+h(x)\right)^{-2},
$$

where $h(x)=O\left(|x|^{-2}\right)$ as claimed. The same arguments easily show that $|\nabla h(x)|=O\left(|x|^{-3}\right)$ and $\Delta h(x)=O\left(|x|^{-4}\right)$ as well. Note that this expression is valid on $\mathbb{R}^{n} \backslash B_{R}(0)$, for any $R \geq R_{0}+C \geq R_{0}+\left|x_{o}\right|$, where $C=C(n, \Lambda)$. 
1.3. Conformal Transformations of $\left(S^{n}, g_{0}\right)$. The group of conformal transformations of $S^{n}$ is isomorphic to the group $O(n+1,1)$ of linear isometries of Minkowski $(n+2)$ space. If $G: S^{n} \rightarrow S^{n}$ is a nonisometric conformal transformation with precisely two fixed points on $S^{n}$, then we refer to $G$ as a dilation. If the fixed points are antipodal points, say $\pm q$, and the differential $d G_{p}: T_{p} S^{n} \rightarrow T_{G(p)} S^{n}$ is a multiple of the identity at $\pm q$, then we refer to $G$ as a centered dilation. Suppose that $G$ is a centered dilation. We then let $\left|G^{\prime}\right|$ denote the function whose value at $p \in S^{n}$ is the linear stretch factor of $G$ at $p$, that is $|d G(v)|=\left|G^{\prime}(p)\right|$ for any unit vector $v \in T_{p} S^{n}$, and assume that $\left|G^{\prime}(q)\right| \geq\left|G^{\prime}(-q)\right|$ We shall refer to $q$ as the source of $G$ and $-q$ as the sink of $G$. In stereographic coordinates we have $\pi^{-1}(-q)=0 \in \mathbb{R}^{n}$, and $G$ has the form

$$
G(x)=\mu x
$$

where $\mu=\left|G^{\prime}(0)\right|$. It is easy to see that $\left|G^{\prime}(q)\right|=\mu^{-1}$. This shows that $\left|G^{\prime}(q)\right|\left|G^{\prime}(-q)\right|=1$, moreover $\mu=1$ if and only if $\left|G^{\prime}\right| \equiv 1$, in which case $G$ is an isometry of $S^{n}$.

Let $\lambda>0$, be defined by $\lambda=\left|G^{\prime}(q)\right|$, and thus $\lambda^{-1}=\mu=\left|G^{\prime}(-q)\right|<1$. We will think of $\lambda$ as a large number (i.e. we will only work with strong dilations), and define a related small quantity $\epsilon$ by $\epsilon=2 \lambda^{-1 / 2}$.

The hypersurface in $S^{n}$ upon which $\left|G^{\prime}(p)\right|=1$, corresponds under stereographic projection to the set $\Sigma_{1}$ of $x \in \mathbb{R}^{n}$, satisfying

$$
\mu\left(1+|x|^{2}\right)=1+\mu^{2}|x|^{2} .
$$

It then follows easily that

$$
\Sigma_{1}=\left\{x:|x|=\lambda^{1 / 2}\right\} .
$$

A general dilation $G$ has a unique decomposition $G=R D$, where $R$ is a rotation and $D$ is a centered dilation. Note that $G^{*} g_{0}=D^{*} R^{*} g_{0}=D^{*} g_{0}$, so that $\left|G^{\prime}\right| \equiv\left|D^{\prime}\right|$. We let $q$ and $-q$ denote the source and sink of $D$, respectively, and $\lambda$ and $\epsilon$ are given by $\lambda=\left|D^{\prime}(q)\right|$, and $\epsilon=2 \lambda^{-1 / 2}$.

1.4. The Bubble Construction. Given a metric $g$ on $M$ such that $R(g)=$ $n(n-1)$ and $g$ is locally conformally flat in a neighborhood of a point $x_{0}$, we now construct a metric $g_{1}$ conformal to $g$ which agrees with $G^{*}\left(g_{0}\right)$ near $x_{0}$, 
and with $g$ away from $x_{0}$. This new metric will have scalar curvature which is bounded and differs from $n(n-1)$ on a set whose volume with respect to $g_{1}$ is small. This is a generalization of [15, Prop. 1.1], which established this result in the case that $(M, g)=\left(S^{n}, g_{0}\right)$.

Proposition 1.2. Let $(M, g)$ be given such that $R(g)=n(n-1)$ and $g$ is conformally flat in a neighborhood of $x_{0} \in M$, with $\Phi$ and $\Lambda$ as defined above. Suppose $G$ is a dilation with source $q=\Phi\left(x_{0}\right)$ and $G=R D$. Let $\lambda$ and $\epsilon$ be as above, and let $\sigma=2 \epsilon^{-1}$. There exists a metric $g_{1}=u^{\frac{4}{n-2}} g$ on $M$ with the following properties:

(i) Let $\epsilon_{1}=\epsilon^{1+\frac{2}{n}}$ and $\epsilon_{2}=\epsilon^{1-\frac{2}{n}}$. The metric $g_{1}$ is equal to $G^{*}\left(g_{0}\right)$ in $B_{c \epsilon_{1}}\left(x_{0}\right)$, and is equal to $g$ on $M \backslash B_{c \epsilon_{2}}\left(x_{0}\right)$, for some constant $c=c(\Lambda)$.

(ii) Let $R_{1}$ denote the scalar curvature function of $g_{1}$. There exists a constant $C$ depending only on $n$ and $\Lambda$, such that the following inequalities hold:

$$
\begin{gathered}
\max \left\{\left|R_{1}(p)\right|: p \in M\right\} \leq C, \\
\operatorname{Vol}_{g_{1}}\left\{p: R_{1}(p) \neq n(n-1)\right\} \leq C \epsilon^{n-2},
\end{gathered}
$$

where $\operatorname{Vol}_{g_{1}}(\cdot)$ denotes the volume taken with respect to $g_{1}$.

(iii) The metric $g_{1}$ can be described as follows near $\partial B_{\epsilon}(q) \subset S^{n}$. Let $x=\left(x^{i}, \cdots, x^{n}\right)$ be stereographic coordinates on $S^{n} \backslash\{q\}$. For $|x|$ near $\sigma, g_{1}$ is given by $g_{1}=4 v_{1}^{\frac{4}{n-2}} \sum_{i=1}^{n}\left(d x^{i}\right)^{2}$, where

$$
v_{1}(x)=\sigma^{2-n}+|x|^{2-n} \text {. }
$$

In particular, $R_{1} \equiv 0$ near $\partial B_{\epsilon}(q)$.

(iv) The metric $g_{1}$ varies continuously under deformations of $G$.

Proof. Since $(M, g)$ is locally conformally flat in a neighborhood of $x_{0}$, there exists an $r>0$ such that $B_{r}\left(x_{0}\right)$, the geodesic ball of radius $\mathrm{r}$ with respect to $g$, is locally conformally flat, i.e. there exists a conformal embedding

$$
\Phi:\left(B_{r}\left(x_{0}\right), g\right) \longrightarrow\left(S^{n}, g_{0}\right)
$$

with $\Phi\left(x_{0}\right)=q \in S^{n}$.

As was shown above, there exists translated stereographic coordinates

$$
\pi \cdot \ell_{-x_{0}}: \mathbb{R}^{n} \rightarrow S^{n} \backslash\{q\}
$$


so that $g$ has an expression of the form

$$
g=4 \bar{v}(x)^{\frac{4}{n-2}} \sum_{i=1}^{n}\left(d x^{i}\right)^{2},
$$

for some $\bar{v}(x)$ as in (1.3). There exists an $R>>1$ such that this expression for $g$ is valid in $\mathbb{R}^{n} \backslash B_{R}(0) \subset \pi^{-1}\left(\Phi\left(B_{r}\left(x_{0}\right)\right)\right)$.

Our new metric will be of the form $g_{1}=4 v_{1}^{\frac{4}{n-2}} \sum_{i=1}^{n}\left(d x^{i}\right)^{2}$ on $\mathbb{R}^{n} \backslash B_{R}(0)$, for some $v_{1} \in C^{\infty}\left(\mathbb{R}^{n} \backslash B_{R}(0)\right), v_{1}>0$ which we shall now construct explicitly. The function $v_{1}$ shall satisfy

$$
v_{1}(x)= \begin{cases}\bar{v}(x) & \text { for } R \leq|x| \leq \sigma_{2} \\ \sigma^{2-n}+\left(a_{0}^{1 / 2}|x|\right)^{2-n} & \text { for } 4 \sigma_{2} \leq|x| \leq \sigma_{1} \\ \left(\lambda\left(1+\lambda^{-2}|x|^{2}\right)\right)^{\frac{(2-n)}{2}} & \text { for } 4 \sigma_{1} \leq|x|\end{cases}
$$

where $\sigma_{1}$ and $\sigma_{2}$ are to be chosen so that $\sigma_{2}<<\sigma<<\sigma_{1}$.

We shall require that

$$
\left(\tan \frac{1}{2} \epsilon_{2}\right)^{-1} \leq \sigma_{2}, \quad \sigma_{1} \leq \frac{1}{4}\left(\tan \frac{1}{2} \epsilon_{1}\right)^{-1}, \quad R \leq\left(\tan \frac{1}{2} \epsilon_{2}\right)^{-1} .
$$

The third inequality will be satisfied provided $\lambda$ is sufficiently large, i.e. provided that $G$ is a sufficiently strong dilation. These three inequalities guarantee that $g_{1}=G^{*}\left(g_{0}\right)$ on $B_{\epsilon_{1}}(q)$ and $g_{1}=g$ on $\Omega \backslash B_{\epsilon_{2}}(q)$. Moreover we may choose a constant $c=c(n, \Lambda)>0$ such that $B_{c \epsilon_{1}}\left(x_{0}\right) \subset \Phi^{-1}\left(B_{\epsilon_{1}}(q)\right)$ and $\Phi^{-1}\left(\Omega \backslash B_{\epsilon_{2}}(q)\right) \subset M \backslash B_{c \epsilon_{2}}\left(x_{0}\right)$. Thus for this constant $c$, these inequalities will guarantee that property (i) holds.

For $a>0$ we define a patching function $\Psi_{a}(x)$ on $\mathbb{R}^{n}$ by setting $\Psi_{a}(x)=$ $\Psi\left(a^{-1}|x|\right)$, where $\Psi_{a}(x)$ satisfies

$$
\Psi_{a}(x)= \begin{cases}0 & \text { for }|x| \leq a \\ 1 & \text { for }|x| \geq 2 a\end{cases}
$$

and $a\left|\nabla \Psi_{a}\right|+a^{2}\left|\nabla \nabla \Psi_{a}\right| \leq C$.

We now define $v_{1}(x)$ explicitly for $\sigma_{2} \leq|x| \leq 4 \sigma_{2}$ and calculate the scalar curvature of the corresponding metric, $g_{1}=4 v_{1}(x)^{\frac{4}{n-2}} \sum_{i=1}^{n}\left(d x^{i}\right)^{2}$. Define

$$
v_{1}(x)= \begin{cases}|x|^{2-n}\left(a_{0}+\left(1-\Psi_{\sigma_{2}}(x)\right) h(x)\right)^{\frac{2 n}{2}} & \text { for } \sigma_{2} \leq|x| \leq 2 \sigma_{2} \\ \Psi_{2 \sigma_{2}}(x) \sigma^{2-n}+\left(a_{0}^{1 / 2}|x|\right)^{2-n} & \text { for } 2 \sigma_{2} \leq|x| \leq 4 \sigma_{2} .\end{cases}
$$


The scalar curvature of $g_{1}=4 v_{1}^{\frac{4}{n-2}} \sum_{i=1}^{n}\left(d x^{i}\right)^{2}$ is given by

$$
R_{1}=-\frac{n-1}{n-2} v_{1}^{-\frac{n+2}{n-2}} \Delta v_{1}
$$

To show that $R_{1}$ is bounded for $\sigma_{2} \leq|x| \leq 2 \sigma_{2}$, we write

$$
v_{1}(x)=|x|^{2-n} f(x)^{\frac{2-n}{2}}
$$

where

$$
f(x)=a_{0}+\left(1-\Psi_{\sigma_{2}}(x)\right) h(x) .
$$

A straightfoward calculation yields the formula

$$
\begin{aligned}
R_{1}(x)=-\frac{n-1}{4}|x|^{3}\left[|x|\left(n|\nabla f(x)|^{2}-2 f(x) \Delta f(x)\right)\right. & \\
& +4(n-2) f(x)(\nabla f(x) \cdot \nabla|x|)] .
\end{aligned}
$$

Using the bounds noted above on $\Psi(x), h(x)$ and their derivatives, it follows immediately that $R_{1}(x)$ is bounded for $\sigma_{2} \leq|x| \leq 2 \sigma_{2}$, for any $\sigma_{2} \geq R \geq 1$. On $2 \sigma_{2} \leq|x| \leq 4 \sigma_{2}, \Delta v_{1}(x)=\sigma^{2-n} \Delta \Psi_{2 \sigma_{2}}$, so we have $\left|\Delta \Psi_{2 \sigma}\right| \leq c \sigma^{n-2}\left(2 \sigma_{2}\right)^{-2}$. A lower bound for $v_{1}(x)$ here, implies $v_{1}(x)^{-\frac{n+2}{n-2}} \leq\left(a_{0}^{1 / 2}|x|\right)^{n+2} \leq c \sigma_{2}^{n+2}$, where $c=c\left(n, a_{0}\right)$. These bounds yield $\left|R_{1}(x)\right| \leq c \sigma^{2-n} \sigma_{2}^{n}$, which is bounded provided $\sigma_{2} \leq c \sigma^{1-\frac{2}{n}}$. Since $\epsilon_{2}=\epsilon^{1-\frac{2}{n}}$ and $\left|\sigma\left(\tan \frac{1}{2} \epsilon\right)^{-1}\right| \leq c \epsilon$, we can choose a constant $c>0$ so that this holds without violating (1.4).

For $\sigma_{1} \leq|x| \leq 2 \sigma_{1}$ we define $v_{1}(x)$ by

$$
v_{1}(x)=\sigma^{2-n}+\left(1-\Psi_{\sigma_{1}}\right)\left(a_{0}^{1 / 2}|x|\right)^{2-n} .
$$

We then get the estimate $\left|R_{1}(x)\right| \leq c \sigma^{n+2} \sigma_{1}^{-n}$, which is bounded provided that $\sigma_{1} \geq c \sigma^{1+\frac{2}{n}}$. As above, since $\epsilon_{1}=\epsilon^{1+\frac{2}{n}}$ we may choose a constant $c>0$ so that this holds without violating (1.4).

Since $\sigma=\lambda^{1 / 2}$, we may rewrite the definition of $v_{1}(x)$ for $|x| \leq 4 \sigma_{1}$, as

$$
v_{1}(x)=\sigma^{2-n}\left(1+\lambda^{-2}|x|^{2}\right)^{\frac{2-n}{2}} .
$$

If we expand this out we may then write

$$
v_{1}(x)=\sigma^{2-n}+\alpha(x),
$$

and for $2 \sigma_{1} \leq|x| \leq 4 \sigma_{1}$ one can easily check that the following bounds on $\alpha(x)$ hold

$$
|\alpha(x)|+\sigma_{1}|\nabla \alpha(x)|+\sigma_{1}^{2}|\nabla \nabla \alpha(x)| \leq c \sigma^{-2-n} \sigma_{1}^{2},
$$


where $\mathrm{c}=\mathrm{c}(\mathrm{n})$. With this in mind we define $v_{1}(x)$ for $2 \sigma_{1} \leq|x| \leq 4 \sigma_{1}$ by

$$
v_{1}(x)=\sigma^{n-2}+\Psi_{2 \sigma_{1}}(x) \alpha(x)
$$

An easy calculation using the bounds above then shows that $\left|R_{1}(x)\right|$ is bounded for $2 \sigma_{1} \leq|x| \leq 4 \sigma_{1}$.

This completes the definition of $g_{1}$ on $\mathbb{R}^{n} \backslash B_{R}(0)$. Clearly by the construction of $v_{1}, g_{1}$ extends to a metric on all of $M$ which agrees with $g$ in $M \backslash B_{c \epsilon_{2}}\left(x_{0}\right)$ and with $G^{*}\left(g_{0}\right)$ in $B_{c \epsilon_{1}}\left(x_{0}\right)$ and satisfies $\left|R_{1}(x)\right| \leq C$ on all of M. Moreover properties (iii) and (iv) can be verified immediately from the construction of $g_{1}$. It remains to verify the second inequality of property (ii), which is equivalent to the inequality

$$
\int_{\left\{x: \sigma_{2} \leq|x| \leq 4 \sigma_{1}\right\}} v_{1}(x)^{\frac{2 n}{n-2}} d x \leq C \epsilon^{n-2} .
$$

For $\sigma_{2} \leq|x| \leq \sigma, v_{1}(x) \leq C|x|^{2-n}$, so that $v_{1}(x)^{\frac{2 n}{n-2}} \leq C|x|^{-2 n}$. Hence

$$
\int_{\left\{x: \sigma_{2} \leq|x| \leq \sigma\right\}} v_{1}(x)^{\frac{2 n}{n-2}} d x \leq C \int_{\left\{x: \sigma_{2} \leq|x| \leq \sigma\right\}}|x|^{-2 n} d x \leq C \sigma_{2}^{-n} \leq C \epsilon^{n-2} .
$$

For $\sigma \leq|x| \leq 4 \sigma_{1}, v_{1}(x) \leq c \sigma^{2-n}$, so that

$$
\int_{\left\{x: \sigma \leq|x| \leq 4 \sigma_{1}\right\}} v_{1}(x)^{\frac{2 n}{n-2}} d x \leq C \int_{\left\{x: \sigma \leq|x| \leq 4 \sigma_{1}\right\}} \sigma^{-2 n} d x \leq C \sigma^{-2 n} \sigma_{1}^{n} .
$$

Since $\sigma_{1} \leq \frac{1}{4}\left(\tan \frac{1}{2} \epsilon_{1}\right)^{-1}$ and $\left|\epsilon_{1}^{1}-\left(\tan \frac{1}{2} \epsilon_{1}\right)^{-1}\right| \leq c \epsilon_{1}$, we can choose a constant $c>0$ such that $\sigma^{-2 n} \sigma_{1}^{n} \leq c \epsilon^{n-2}$. Thus $\operatorname{Vol}_{g_{1}}\left\{x: R_{1}(x) \neq n(n-1)\right\} \leq c \epsilon^{n-2}$. This completes the proof of Proposition 1.2.

1.5. Conformal Structures and Approximate Solutions. Our $N$ approximate solutions $g_{1_{1}}, \ldots, g_{1_{N}}$ can be distinguished from each other by the number of spherical regions (defined below) that each one possesses, for example, $g_{1_{k}}$ will have $k$ spherical regions. We will first show how to construct an approximate solution with $k$ spherical regions, for any $k \geq 1$. Once this is done it will be easy to construct a conformal class with $\mathrm{N}$ approximate solutions.

The conformal data which we will use to construct our approximate solution consists of a chain $\mathcal{T}_{k}$, of $k$ vertices $(1, \ldots, k)$, each of which is labeled with a conformal transformation of $S^{n}, F_{i}$ for $1 \leq i \leq k$. We will denote our maps either with subscripts $i$, corresponding to the vertex $i$, or with subscripts $\pm i$, 
$+i$ denoting the edge between $i$ and $i+1$ and $-i$ denoting the edge between $i$ and $i-1$. We require that the transformations satisfy, for $2 \leq i \leq k-1$,

$$
G_{ \pm i}=F_{i \pm 1} \cdot F_{i}^{-1}=R_{ \pm i} D_{ \pm i}
$$

and for $i=1$ and $i=k$

$$
G_{+1}=F_{2} \cdot F_{1}^{-1}=R_{+1} D_{+1} \text { and } G_{-k}=F_{k-1} \cdot F_{k}^{-1}=R_{-k} D_{-k}
$$

are strong dilations whose sources are sufficiently separated for each $i, 2 \leq$ $i \leq k-1$ (this will be made precise momentarily). We also associate to each terminal vertex another such dilation which we denote

$$
G_{-1}=R_{-1} D_{-1} \text { and } G_{+k}=R_{+k} D_{+k} .
$$

Thus for $1 \leq i \leq k$, we have associated to each edge $\pm i$, corresponding dilations $G_{ \pm i}$. We let $q_{ \pm i}$ denote the source of $G_{ \pm i}$, and $\lambda_{ \pm i}=\left|G_{ \pm i}^{\prime}\left(q_{ \pm i}\right)\right|=\left|D_{ \pm i}^{\prime}\left(q_{ \pm i}\right)\right|$ denote its strength. As before we also define $\epsilon_{ \pm i}=2 \lambda_{ \pm i}^{-\frac{1}{2}}$. This conformal data will be admissible provided that there are constants $\beta>0$ and $\epsilon>0$ such that, for every $i$,

$$
\begin{array}{r}
\left|q_{+i}-q_{-i}\right| \geq \beta^{-1} \\
\beta^{-1} \epsilon \leq \epsilon_{ \pm i} \leq \beta \epsilon .
\end{array}
$$

Following [15], such a labeling of the chain $\mathcal{T}_{k+1}$ will be called an admissible conformal structure, and is denoted by $\sigma$.

We will now define using the conformal data above, a domain $\Omega \subset S^{n}$ which will be composed of $k$ almost spherical regions, $\Omega_{1}, \ldots, \Omega_{k}$. For each $i \in[1, k]$, let $B_{ \pm i}$ be the small ball such that

$$
\partial B_{ \pm i}=S_{ \pm i}=\left\{p:\left|G_{ \pm i}(p)\right|=1\right\} .
$$

Since we are assuming that $q_{+i}$ and $q_{-i}$ are sufficiently separated and that $G_{+i}$ and $G_{-i}$ are strong dilations, it follows that $B_{+i}$ and $B_{-i}$ are disjoint. Thus we may let $\mathcal{O}_{i}=S^{n} \backslash\left\{B_{+i} \cup B_{-i}\right\}$ and define

$$
\Omega_{i}=F_{i}^{-1}\left(\mathcal{O}_{i}\right) .
$$


By their definitions $G_{-i+1}=G_{+i}^{-1}$, thus, since $\left|G^{-1^{\prime}}(G(p))\right|=\left|G^{\prime}(p)\right|^{-1}$ we have

$$
\left|G_{-i+1}^{\prime}\left(G_{+i}(p)\right)\right|=\left|G_{+i}^{\prime}(p)\right|^{-1} .
$$

Now if we observe that $S^{n} \backslash B_{-i+1}=\left\{p:\left|G_{-i+1}^{\prime}(p)\right|<1\right\}$ and $B_{+i}=\{p$ : $\left.\left|G_{+i}^{\prime}(p)\right| \geq 1\right\}$, then since

$$
\left|G_{+i}^{\prime}\left(G_{-i+1}\left(S^{n} \backslash B_{-i+1}\right)\right)\right|=\left|G_{-i+1}^{\prime}\left(S^{n} \backslash B_{-i+1}\right)\right|^{-1},
$$

we have

$$
G_{-i+1}\left(S^{n} \backslash B_{-i+1}\right)=\bar{B}_{+i}
$$

Therefore $F_{i}\left(\Omega_{i+1}\right) \subset B_{+i}$ which implies that $F_{i}\left(\Omega_{i+1}\right) \cap \mathcal{O}_{i}=\emptyset$. Applying $F_{i}^{-1}$ then shows that

$$
\Omega_{i+1} \cap \Omega_{i}=\emptyset
$$

Combining this with the above equality gives us

$$
\bar{\Omega}_{i} \cap \bar{\Omega}_{i+1}=F_{i}^{-1}\left(S_{+i}\right)=F_{i+1}^{1}\left(S_{-i+1}\right),
$$

and moreover, we clearly have $\bar{\Omega}_{i} \cap \bar{\Omega}_{j}=\emptyset$ for any $i, j$ such that $|i-j| \geq 2$. Thus we may define the open set $\Omega \subset S^{n}$ by

$$
\Omega=\bigcup_{i=1}^{k} \Omega_{i}
$$

We now construct a metric $g_{1}$ on $\Omega$ in such a way that each $\left(\Omega_{i}, g_{1}\right)$ will be an almost spherical region, or asr for short. This will mean that outside of a small neighborhood of its two boundary components $\Omega_{i}$ will be isometric to $S^{n}$ with two small disjoint disks removed. We first define a metric $g_{i}$ on $\mathcal{O}_{i}$ which will be used to define $g_{1}$ on $\Omega_{i}$. Let $g_{+i}$ (respectively $g_{-i}$ ) denote the metric associated to the dilation $G_{+i}$ (respectively $G_{-i}$ ) by Proposition 1.2 , applied to the manifold $(M, g)=\left(S^{n}, g_{0}\right)$, with $\Phi \equiv$ the identity map on $S^{n}$. Since $g_{+i}$ and $g_{-i}$ both agree outside of two small disjoint balls, we can define a new metric $g_{i}$ on $S^{n}$ by

$$
g_{i}= \begin{cases}g_{+i} & \text { in } B_{\delta}\left(q_{+i}\right) \\ g_{-i} & \text { in } B_{\delta}\left(g_{-i}\right) \\ g_{0} & \text { in } S^{n} \backslash\left\{B_{\delta}\left(q_{+i}\right) \cup B_{\delta}\left(q_{-i}\right)\right\}\end{cases}
$$


where $\delta$ is a small radius, larger than $\epsilon_{+i}^{1-2 / n}$ and $\epsilon_{-i}^{12 / n}$. We then define $g_{1}$ in $\Omega_{i}$ by

$$
g_{1}=F_{i}^{*}\left(g_{i}\right) \text { on } \Omega_{i}=F_{i}^{-1}\left(\mathcal{O}_{i}\right) .
$$

We must show that this definition fits together to give us a smooth metric on all of $\Omega$. This is expressed in the compatibility conditions

$$
\begin{aligned}
& F_{i}^{*}\left(g_{i}\right)=F_{i+1}^{*}\left(g_{i+1}\right) \quad \text { near } F_{i}^{-1}\left(S_{+i}\right)=F_{i+1}^{-1}\left(S_{-i+1}\right) \\
& F_{i}^{*}\left(g_{i}\right)=F_{i-1}^{*}\left(g_{i-1}\right) \quad \text { near } F_{i}^{-1}\left(S_{-i}\right)=F_{i 1}^{-1}\left(S_{+i-1}\right)
\end{aligned}
$$

or equivalently,

$$
\begin{aligned}
& g_{i}=G_{+i}^{*}\left(g_{i+1}\right) \text { near } S_{+i} \\
& g_{i}=G_{-i}^{*}\left(g_{i-1}\right) \text { near } S_{-i} \text {. }
\end{aligned}
$$

To see that $g_{i}$ extends smoothly across $\partial \bar{\Omega}_{i} \cap \partial \bar{\Omega}_{i+1}=F_{i}^{-1}\left(S_{+i}\right)=F_{i+1}^{-1}\left(S_{-i+1}\right)$, recall that near $S_{+i}$ in $\mathcal{O}_{i}$, we have

$$
g_{i}=g_{+i}=4 v_{+i}^{\frac{4}{n-2}}(x) \sum_{j=1}^{n}\left(d x^{j}\right)^{2},
$$

in stereographic coordinates on $S^{n} \backslash q_{+i}$, where $v_{+i}(x)=\sigma_{+i}^{2-n}+|x|^{2-n}$. Since $S_{+i}=G_{+i}^{-1}\left(S_{-i+1}\right)$, the metric $G_{+i}^{*}\left(g_{i+1}\right)$ near $S_{+i}$ in $\mathcal{O}_{i}$, is the same as $g_{i+1}$ near $S_{-i+1}$ in $\mathcal{O}_{i+1}$. Near $S_{-i+1}$ in $\mathcal{O}_{i+1}$, we have

$$
g_{i+1}=g_{-i+1}=4 v_{-i+1}^{\frac{4}{n-2}}(y) \sum_{j=1}^{n}\left(d y^{j}\right)^{2}
$$

in stereographic coordinates on $S^{n} \backslash q_{-i+1}$, where $v_{-i+1}(y)=\sigma_{-i+1}^{2-n}+|y|^{2-n}$. Finally, since $G_{+i}=G_{-i+1}^{1},-q_{+i}=q_{-i+1}$ and $\sigma_{+i}^{-1}=\sigma_{-i+1}$, we see that we have two different coordinate descriptions for the same metric. The argument above clearly applies near $S_{-i}$ as well, so we have shown that $g_{1}$ extends to a smooth metric on all of $\Omega$, and, by construction, each $\Omega_{i}$ is an asr, the isometry being given by $F_{i}$.

To complete our construction of an approximate solution, we must attach $(M, g)$ to one end of $\Omega$, and $\left(S^{n}, \bar{g}_{0}\right)$ to the other. Recall both $g$ and $\bar{g}_{0}$ are metrics whose conformal classes are nondegenerate and which are conformally flat in the neighborhood of some point. Since $(M, g)$ is conformally flat in a neighborhood of $x_{0}$, we have a conformal map

$$
\Phi:\left(B_{r}\left(x_{0}\right), g\right) \rightarrow\left(S^{n}, g_{0}\right)
$$


for some $r>0$. By applying Proposition 1.2 to $(M, g)$ with the dilation $G=G_{-1}^{-1}$ whose source is $-q_{-1}=\Phi\left(x_{0}\right)$ we have a metric $\bar{g}$ on $M$ which satisfies each of the properties $(i)-(i v)$. Let $B_{0}=G_{-1}\left(S^{n} \backslash B_{-1}\right)$ be the small ball such that $\partial B_{0}=G_{-1}\left(S_{-1}\right)=S_{+0}=\left\{p:\left|G_{-1}^{-1^{\prime}}(p)\right|=1\right\}$. We define $M_{0}$ by

$$
M_{0}=M \backslash \Phi^{-1}\left(B_{0}\right) .
$$

We attach $\left(M_{0}, \bar{g}\right)$ to $\left(\Omega, g_{1}\right)$ as follows. Define a new conformal transformation of $S^{n}, F_{0}$ by $F_{0}=G_{-1} \cdot F_{1}$. We extend the definition of $g_{1}$ as follows. Let $g_{1}=F_{0}^{*}(\bar{g})$ on $F_{0}^{-1}\left(\left(S^{n} \backslash B_{0}\right) \cap \Phi\left(B_{r}\left(x_{0}\right)\right)\right)$ and let

$$
\Omega^{+}=\Omega \cup F_{0}^{-1}\left(\left(S^{n} \backslash B_{0}\right) \cap \Phi\left(B_{r}\left(x_{0}\right)\right)\right) .
$$

From the construction of $\bar{g}$ on $\left(S^{n} \backslash B_{0}\right) \cap \Phi\left(B_{r}\left(x_{0}\right)\right)$, of $g_{1}$ on $\Omega$, we see as above, that $g_{1}$ thus defined extends smoothly across $\partial \Omega \cap F_{0}^{1}\left(S_{-1}\right)$ into $\Omega$. Moreover, the map $F_{0}^{-1} \cdot \Phi$ is an isometry from a neighborhood of $\partial M_{0} \subset$ $\left(M_{0}, \bar{g}\right)$ onto a neighborhood of $\partial \Omega \cap F_{0}^{-1}\left(S_{-1}\right) \subset\left(\Omega, g_{1}\right)$. Thus we have a manifold diffeomorphic to $M \backslash B_{r_{0}}\left(x_{0}\right)$, having $k$ asr's and which we write as

$$
M_{0} \cup_{F_{0}} \Omega \text {. }
$$

To form the closed manifold $\left(M, g_{1}\right)$ we attach $\left(S_{0}^{n}, \bar{g}_{0}\right)$ to the end of $\Omega$ represented by the terminal vertex $k$. This is done as above by applying Proposition 1.2 to $\left(S^{n}, \bar{g}_{0}\right)$ with the dilation $G=G_{+k}^{-1}$ whose source is $-q_{+k}=\Phi\left(x_{0}\right)$, for $x_{0} \in S^{n}$. This gives us a closed manifold $M$, and a smooth metric $g_{1}$ on $M$ which has $k$ asr's and a nondegenerate spherical cap. We can represent this decomposition of $\left(M, g_{1}\right)$ by writing

$$
M=M_{0} \cup_{F_{0}} \Omega \cup_{F_{k+1}} S_{0}^{n} .
$$

We end this section with an observation concerning the volumes of our approximate solutions which follows immediately from the construction. This will allow us to give precise estimates for the energies of the solutions we construct. Let $V_{1}=\operatorname{Vol}_{g}(M), V_{2}=\operatorname{Vol}_{\bar{g}_{0}}\left(S^{n}\right)$ and $\omega_{n}=\operatorname{Vol}_{g_{0}}\left(S^{n}\right)$, then for some $c=c\left(n, g, \bar{g}_{0}\right)$

$$
\left|\operatorname{Vol}_{g_{1_{k}}}(M)-\left(V_{1}+V_{2}+k \omega_{n}\right)\right| \leq c \epsilon^{n-2} .
$$


1.6. A Conformal Class With $N$ Approximate Solutions. The construction of $N$ distinct approximate solutions $\left(g_{1_{1}}, \ldots, g_{1_{N}}\right)$, having $1, \ldots, N$ asr's respectively, is based upon the fact that an annular region

$$
A=\{x: 1<|x|<R\}
$$

can be decomposed into $k$ subannuli, for each $k=1, \ldots, N$, so that each subannulus has conformal modulus $R^{1 / k}$. The conformal modulus, $c m(A)$, of an annulus is defined to be the ratio of the outer radius to the inner radius. Two annuli are conformally equivalent if and only if they have the same conformal modulus. The construction of the $N$ approximate solutions will be done using appropriate powers of a fixed centered dilation $G$. The powers will be chosen so that each of the subdomains, $\Omega_{i}^{k}$ corresponding to the decomposition of a fixed domain $\Omega \subset S^{n}$ into $k$ subdomains, as done above, will have conformal modulus (in stereographic coordinates)

$$
\operatorname{cm}\left(\pi^{-1}\left(\Omega_{i}^{k}\right)\right)=R^{1 / k}
$$

where $R=c m\left(\pi^{-1}(\Omega)\right)$. Note that the metrics $g_{1_{k}}$ constructed from these conformal structures will be spherically symmetric on $\pi^{-1}(\Omega)$.

We fix $R>>1$ and let $G$ be a centered dilation with source $q \in S^{n}$ and strength $\lambda=\left|G^{\prime}(q)\right|=R$. For any $k, 1 \leq k \leq N$, we let for each $i, 1 \leq i \leq k$,

$$
\begin{aligned}
& G_{-i}=G^{1 / k} \\
& G_{+1}=G^{-1 / k} .
\end{aligned}
$$

If $k$ is even, we set $F_{i}=G^{\frac{k-2 i+1}{2 k}}$ for $1 \leq i \leq k / 2$, and $F_{i}=F_{k-i+1}^{-1}$ for $1+k / 2 \leq i \leq k$. If $k$ is odd we set $F_{i}=G^{\frac{k-2 i+1}{2 k}}$ for $1 \leq i \leq \frac{k-1}{2}, F_{i}=F_{k-i+1}^{-1}$ for $\frac{k+3}{2} \leq i \leq k$ and $F_{\frac{k+1}{2}}=$ the identity. It is easily checked that this then defines a conformal structure which satisfies

$$
\operatorname{cm}\left(\pi^{-1}\left(\Omega_{i}^{k}\right)\right)=R^{1 / k}
$$

for each $i, 1 \leq i \leq k$.

Let $g_{1_{k}}$ denote the metric constructed canonically from the conformal structure above. Each $\left(\Omega_{i}^{k}, g_{1_{k}}\right)$ is conformally equivalent to a Euclidean annulus. We define $c m\left(\Omega_{i}^{k}, g_{1_{k}}\right)$ to be the conformal modulus of this Euclidean annulus. 
It is immediate from the construction of $g_{1_{k}}$ that for $1 \leq i, j \leq k$

$$
\left(\Omega_{i}^{k}, g_{1_{k}}\right) \cong\left(\Omega_{j}^{k}, g_{1_{k}}\right)
$$

where $\cong$ denotes conformal equivalence. Finally the fact that for each $k$,

$$
c m\left(\pi^{1}(\Omega)\right)=\prod_{i=1}^{k} c m\left(\pi^{-1} \Omega_{i}^{k}\right)=\prod_{i=1}^{k} R^{1 / k}=R,
$$

allows us to conclude that

$$
c m\left(\Omega, g_{1_{k}}\right)=\prod_{i=1}^{k} c m\left(\Omega_{i}^{k}, g_{1_{k}}\right) .
$$

and that the right hand side is independent of $\mathrm{k}$. Thus each $\left(\Omega, g_{1_{k}}\right)$ has the same conformal modulus, independent of the metric $g_{1_{k}}$. Extending these metrics to approximate solutions on all of $M$ as in (1.7) we easily see that $g_{1_{1}}, \ldots, g_{1_{k}}$ all lie in the same conformal class. Any collection of admissible conformal structures $\sigma_{1}, \ldots, \sigma_{N}$ on chains $\mathcal{T}_{1}, \ldots, \mathcal{T}_{N}$ for which the metrics $g_{1_{1}}, \ldots, g_{1_{k}}$ all lie within the same conformal class, will be called an admissible $N$ structure and will be denoted by $\sigma(N)$. Note that each $g_{1_{k}}$ is uniquely determined from $\left(\mathcal{T}_{k}, \sigma_{k}\right)$ by the construction given above.

We close this section with a summary of our construction.

Theorem 1.3. Given an integer $N \geq 1$ and metrics $g$ on $M$, and $\bar{g}_{0}$ on $S^{n}$, such that $R(g)=R\left(\bar{g}_{0}\right)=n(n-1)$, there exists a constant $c$ depending only on $n, \beta, g, \bar{g}_{0}$ and a admissible $N$ structure $\sigma(N)$ with metrics $g_{1_{1}}, \ldots, g_{1_{N}}$ such that, for each $k$

$$
\begin{aligned}
\max \left\{x \in M:\left|R\left(g_{1_{k}}\right)\right|(x)\right\} & \leq c \\
\operatorname{Vol}\left\{x \in M: R\left(g_{1_{k}}\right)(x) \neq n(n-1)\right\} & \leq c \epsilon^{n-2} \\
\left|\operatorname{Vol}_{g_{1_{k}}}(M)-\left(V_{1}+V_{2}+k \omega_{n}\right)\right| & \leq c \epsilon^{n-2} .
\end{aligned}
$$

Moreover, $\left(M, g_{1_{k}}\right)$ has a decomposition

$$
M=M_{0} \cup_{F_{0}} \Omega^{k} \cup_{F_{k+1}} S_{0}^{n}
$$

where $g_{1_{k}}$ has the explicit description in $\Omega^{k}$ given above. 


\section{The Linear Analysis}

2.1. The Linearized Equation. Associated to each admissible conformal structure $\sigma$ on $\mathcal{T}_{k}$, there is a uniquely determined approximate solution, $g_{1_{k}}$, constructed in section 2. We refer to $\left(\mathcal{T}_{k}, \sigma\right)$, or just $\sigma$ as an approximate solution. We define $[\sigma]$ to be the conformal class of any metric $g_{1_{k}}$ constructed from an admissible $N$-structure, $\sigma(N)$. We work with an approximate solution with $k$ asr's, for any $k \in[1, N]$. Since $k$ will be fixed for much of our study, we write $g_{1}$ for $g_{1_{k}}$, omitting the subscript wherever convenient.

We want to find $g \in[\sigma]$ which satisfy $R(g)=n(n-1)$ and are close to $g_{1}$. With this in mind we write

$$
g=(1+\eta)^{\frac{4}{n-2}} g_{1}
$$

regarding $\eta$ as a small perturbation of the approximate solution 1. Equation (1.1) can be written as

$$
\mathcal{L} \eta=\frac{n-2}{4(n-1)}\left(R_{1}-n(n-1)\right)(1+\eta)+Q(\eta)=F(x, \eta),
$$

where $R_{1}=R\left(g_{1}\right), \mathcal{L}=\Delta_{g_{1}}+n$ is the linear operator appearing in (1.2), and

$$
Q(\eta)=\frac{n(n-2)}{4}\left(1+\frac{n+2}{n-2} \eta-(1+\eta)^{\frac{n+2}{n 2}}\right) .
$$

Since we are interested in small $\eta, Q(\eta)$ is quadratically small.

In this section we show that we can find a $\mathrm{k}(\mathrm{n}+1)$ dimensional small eigenspace $K$, consisting of all the $L^{2}(M)$ eigenfunctions of $\mathcal{L}$ with appropriately small eigenvalues. $K$ will be very close, in $L^{2}(M)$, to an approximate kernel $K_{0}$, which we explicitly construct. We then find a bounded inverse of $\mathcal{L}$ off of $K$ and show that we can uniquely solve the linear equation $\mathcal{L} \eta=f$, provided that $f$ and $\eta$ are orthogonal to $K$, and obtain precise $\epsilon$-independent estimates on the solutions. The closeness of $K$ to the explicit space $K_{0}$ is part of what will allow us to identify the component of $F(x, \eta)$ lying in $K$. Inverting $\mathcal{L}$ off of some abstract subspace without having such explicit control would not help us to solve the nonlinear problem.

The idea behind the identification of the small eigenspace is that for $\epsilon$ sufficiently small, the annular region $\Omega^{k}$, composed of $k$ asr's, behaves spectrally like the disjoint union of $k$ standard spheres. As has been pointed out, the 
kernel of $\mathcal{L}$ can be identified explicitly on $S^{n}$ as the span of the linear coordinate functions. It is from these functions that we construct our approximate kernel by carefully cutting off the coordinate functions on each $\mathcal{O}_{i}$ and using $F_{i}$ to pull these functions back to $\Omega_{i}$. The assumption that the metrics $g$ and $\bar{g}_{0}$ on the ends, $M_{0}$ and $S_{0}^{n}$ of $\Omega$, are nondegenerate insures that these ends do not support an approximate kernel for $\mathcal{L}$, in $L^{2}(M)$.

2.2. The Approximate Kernel. We shall construct our approximate kernel by cutting off the coordinate functions in the $k+1$ neck regions, which join the asr's $\Omega_{i}^{k}$ to each other, and to the ends $M_{0}$ and $S_{0}^{n}$. To do this we use a smoothed out harmonic cutoff function, which we find by first solving a Dirichlet problem on each neck region.

For each vertex $i \in[2, k-1]$ there are two neck regions between $\Omega_{i}$ and its two adjacent asr's. Fix $\delta$ to be a small radius which is substantially larger than $\epsilon$, and define

$$
\begin{aligned}
& N_{+i}^{(\delta)}=F_{i}^{-1}\left(B_{\delta}\left(q_{+i}\right)\right) \cap F_{i+1}^{-1}\left(B_{\delta}\left(q_{i+1}\right)\right) \\
& N_{-i}^{(\delta)}=F_{i}^{-1}\left(B_{\delta}\left(q_{-i}\right)\right) \cap F_{i-1}^{-1}\left(B_{\delta}\left(q_{+i 1}\right)\right) .
\end{aligned}
$$

Note that $N_{+i}^{(\delta)}=N_{-i+1}^{(\delta)}$, and $N_{-i}^{(\delta)}=N_{+i-1}^{(\delta)}$. For the terminal vertices 1 and $k, N_{+1}^{(\delta)}$ and $N_{-k}^{(\delta)}$ are defined as above, and the terminal neck regions $N_{-1}^{(\delta)}$ and $N_{+k}^{(\delta)}$ are defined by

$$
\begin{aligned}
& N_{-1}^{(\delta)}=F_{1}^{-1}\left(B_{\delta}\left(q_{-1}\right)\right) \cap F_{0}^{-1}\left(B_{\delta}\left(-q_{1}\right)\right) \\
& N_{+k}^{(\delta)}=F_{k}^{-1}\left(B_{\delta}\left(q_{+k}\right)\right) \cap F_{k+1}^{-1}\left(B_{\delta}\left(-q_{+k}\right)\right)
\end{aligned}
$$

The fact which distinguishes these terminal neck regions from the other $k-1$ neck regions, is that near the boundary component not contained in $\Omega$, the metric $g_{1}$ is not the standard spherical metric $g_{0}$ as it is near both boundary components of the other neck regions. Here $g_{1}$ is isometric to one of the conformally flat metrics $g$ and $\bar{g}_{0}$, respectively. We also note that for each $i \in[1, k]$

$$
\Omega_{i}^{(-\delta)}=\Omega_{i} \backslash\left\{N_{-i}^{(\delta)} \cup N_{+i}^{(\delta)}\right\}
$$

with the metric $g_{1}$, is isometric to the standard sphere with two balls removed

$$
\mathcal{O}_{i}^{(-\delta)}=\left(S^{n} \backslash\left\{B_{\delta}(q-i) \cup B_{\delta}(q+i)\right\}, g_{0}\right)
$$


provided that $\delta>\epsilon_{ \pm i}^{1-\frac{2}{n}}$. Similarly,

$$
M_{0}^{(-\delta)}=M_{0} \backslash N_{-1}^{(\delta)} \quad \text { and } \quad S_{0}^{n(\delta)}=S_{0}^{n} \backslash N_{+k}^{(\delta)}
$$

with the metric $g_{1}$, are isometric to

$$
\left(M \backslash \Phi^{-1}\left(B_{\delta}\left(-q_{-1}\right)\right), g\right) \text { and }\left(S^{n} \backslash \Phi^{-1}\left(B_{\delta}\left(-q_{+k}\right)\right), \bar{g}_{0}\right),
$$

respectively.

Fix a vertex $i, 1 \leq i \leq k$ and a neck region, say $N_{+i}^{(\delta)}$. Let $h_{+i}$ be the solution of the following Dirichlet problem on $N_{+i}^{(\delta)}$.

$$
\begin{array}{rlll}
\Delta_{g_{1}} h_{+i}=0 & \text { on } & N_{+i}^{(\delta)} \\
h_{+i}=1 & \text { on } & F_{i}^{-1}\left(\partial B_{\delta}\left(q_{+i}\right)\right) \\
h_{+i}=0 & \text { on } & F_{i+1}^{-1}\left(\partial B_{\delta}\left(q_{-i+1}\right)\right) .
\end{array}
$$

We choose an orthonormal basis for $\mathbb{R}^{n+1}, \omega_{1}, \ldots, \omega_{n+1}$, and let $q^{\alpha}$ denote the $\alpha$ coordinate function on $S^{n}$ with respect to this basis. Thus for any point $q \in S^{n}$, we write $q=\sum_{\alpha=1}^{n+1} q^{\alpha} \omega_{\alpha}$. For each $i \in[1, k]$, and $\alpha \in[1, n+1]$ we define functions $q_{i}^{\alpha}$ on $S^{n}$ by,

$$
\left(2.4 q_{i}^{\alpha}(q)=\left\{\begin{array}{ccc}
q^{\alpha}+\Psi_{\delta}\left(\rho\left(q, q_{+i}\right)\right)\left(q_{+i}^{\alpha}-q^{\alpha}\right)+\Psi_{\delta}\left(\rho\left(q, q_{-i}\right)\right)\left(q_{-i}^{\alpha}-q^{\alpha}\right) \\
& \text { for } q \in S^{n} \backslash\left\{B_{\delta}\left(q_{+i}\right) \cup B_{\delta}\left(q_{-i}\right)\right\} \\
q_{+i}^{\alpha} \cdot \bar{h}_{+i} \cdot F_{i}^{-1} & \text { for } & q \in B_{\delta}\left(q_{+i}\right) \\
q_{-i}^{\alpha} \cdot \bar{h}_{-i} \cdot F_{i}^{-1} & \text { for } & q \in B_{\delta}\left(q_{-i}\right)
\end{array}\right.\right.
$$

Here $\rho(\cdot, \cdot)$ is the distance function on $\left(S^{n}, g_{0}\right)$, and $\bar{h}_{+i}$ is the function $h_{+i}$, smoothed out to be globally defined as follows

$$
\bar{h}_{+i}=\left\{\begin{array}{lll}
1 & \text { on } & A_{+i}=F_{i}^{-1}\left(B_{\delta}\left(q_{+i}\right) \backslash B_{\frac{3 \delta}{4}}\left(q_{+i}\right)\right) \\
\left(h_{+i}-1\right)\left(\Psi_{+i} \cdot F_{i}\right)+1 & \text { on } & B_{+i}=F_{i}^{-1}\left(B_{\frac{3 \delta}{4}}\left(q_{+i}\right) \backslash B_{\frac{\delta}{2}}\left(q_{+i}\right)\right) \\
h_{+i} & \text { on } & C_{+i}=F_{i}^{-1}\left(B_{\frac{\delta}{2}}\left(q_{+i}\right)\right) \cap F_{i+1}^{-1}\left(B_{\frac{\delta}{2}}\left(q_{-i+1}\right)\right) \\
h_{+i}\left(\Psi_{-i+1} \cdot F_{i+1}\right) & \text { on } & B_{-i+1}=F_{i+1}^{-1}\left(B_{\frac{3 \delta}{4}}\left(q_{-i+1}\right) \backslash B_{\frac{\delta}{2}}\left(q_{-i+1}\right)\right) \\
0 & \text { on } & A_{-i+1}=F_{i+1}^{-1}\left(B_{\delta}\left(q_{-i+1}\right) \backslash B_{\frac{3 \delta}{4}}\left(q_{-i+1}\right)\right)
\end{array}\right.
$$

Here $\Psi_{+i}=\Psi\left(4 \rho\left(\cdot, q_{+i}\right)-\delta\right)$ and $\Psi_{-i+1}=\Psi\left(4 \rho\left(\cdot, q_{-i+1}\right)-\delta\right)$. The function $\bar{h}_{-1}$ is defined similarly about the neck region $N_{-i}^{(\delta)}$. The approximate kernel $K_{0}$ is formed from the functions $q_{i}^{\alpha}$ by defining for $1 \leq i \leq k$ and $1 \leq \alpha \leq n+1$

$$
\eta_{i}^{\alpha}=q_{i}^{\alpha} \cdot F_{i}
$$

and then letting

$$
K_{0}=\text { the linear span of the } \eta_{i}^{\alpha} \text { in } L^{2}(M) .
$$


Note that for each $i, \eta_{i}^{\alpha} \in C^{\infty}(M)$ and has support in $\Omega_{i}^{(+\delta)}$.

In establishing pointwise and $L^{2}$ bounds for $\mathcal{L} \eta_{i}^{\alpha}$ we need to have strong estimates on $h_{+i}$ and $h_{-i}$ and their derivatives near the boundaries of $N_{+i}^{(\delta)}$ and $N_{-i}^{(\delta)}$ respectively. This is expressed by the following Lemma.

Lemma 2.1. The functions $h_{+i}$ (and similarly $h_{-i}$ ) satisfy the bounds

$$
\begin{aligned}
\sup \left\{\left(1-h_{+i}(x)\right)+\delta\left|\nabla h_{+i}(x)\right|: x \in F_{i}^{-1}\left(B_{\delta}\left(q_{+i}\right) \backslash B_{\frac{\delta}{2}}\left(q_{+i}\right)\right)\right\} & \leq c(\epsilon / \delta)^{n-2} \\
\sup \left\{h_{+i}(x)+\delta\left|\nabla h_{+i}(x)\right|: x \in F_{i+1}^{-1}\left(B_{\delta}\left(q_{-i+1}\right) \backslash B_{\frac{\delta}{2}}\left(q_{-i+1}\right)\right)\right\} & \leq c(\epsilon / \delta)^{n-2} .
\end{aligned}
$$

We refer to [15, Lemma 3.1] for the proof, these bounds are established there when $N_{+i}^{(\delta)}$ is a neck region separating two asr's. In the case of the extreme neck regions, e.g. $N_{+k}^{(\delta)}=F_{k}^{-1}\left(B_{\delta}\left(q_{+k}\right) \cap G_{+k}^{-1}\left(B_{\delta}\left(-q_{+k}\right)\right)\right)$, we have an explicit description of $g_{1}$ in stereographic coordinates $x=\left(x^{1}, \ldots, x^{n}\right)$ on $S^{n} \backslash-q_{+k}$. In these coordinates $g_{1}=4 v_{1}^{\frac{4}{n-2}} \sum_{i=1}^{n}\left(d x^{i}\right)^{2}$, and the region above is contained in $\left\{x: R \leq|x| \leq \sigma_{2}\right\}$, where $\sigma=\lambda_{+1}^{1 / 2}$ and $\sigma_{2}$ is defined as in Proposition 1.2. Thus, the conformal factor takes the form of (1.3). A partial description of $v_{1}(x)$ on a larger set is then given by,

$$
v_{1}(x)=\left\{\begin{array}{lll}
|x|^{2-n}\left(a_{0}+h(x)\right)^{\frac{2-n}{2}} & \text { for } \quad R \leq|x| \leq \sigma_{2} \\
\sigma^{2-n}+\left(a_{0}^{1 / 2}|x|\right)^{2-n} & \text { for } \quad 4 \sigma_{2} \leq|x| \leq \sigma_{1}
\end{array}\right.
$$

where $h(x)=O\left(|x|^{-2}\right)$. Consider the Kelvin transform $y=K(x)=\frac{x}{|x|^{2}}$, if we now write $g_{1}=4 w_{1}(y) \sum_{i=1}^{n}\left(d y^{i}\right)^{2}$, then $w_{1}(y)$ is partially given by

$$
w_{1}(y)=\left\{\begin{array}{lll}
\sigma^{2-n}|y|^{2-n}+a_{0}^{\frac{2-n}{2}} & \text { for } \quad \sigma_{1}^{-1} \leq|y| \leq \frac{1}{4} \sigma_{2}^{-1} \\
\left(a_{0}+k(y)\right)^{\frac{2-n}{2}} & \text { for } \quad \sigma_{2}^{-1} \leq|y| \leq R^{-1} .
\end{array}\right.
$$

where $k(y)=O\left(|y|^{2}\right)$. It then follows that $w_{1}(y)$ satisfies

$$
\begin{gathered}
\left|w_{1}(y)-a_{0}^{\frac{2-n}{2}}\right| \leq c\left(|y|^{2}+\epsilon^{n-2}|y|^{2-n}\right), \\
\sum_{i=1}^{n}\left|\frac{\partial w_{1}}{\partial y^{i}}(y)\right| \leq c\left(|y|+\epsilon^{n-2}|y|^{1-n}\right),
\end{gathered}
$$

for $y$ such that $\epsilon \leq|y| \leq R^{-1}$, where $\epsilon=2 \lambda_{+k}^{1 / 2}$. The proof of Lemma 2.1 then follows precisely as in [15, Lemma 3.1]. Lemma 2.1 allows us to prove the relevant pointwise and $L^{2}$ estimates on $K_{0}$, in exactly the same manner as [15, Lemma 3.2]. We refer there for the proof of 
Proposition 2.2. The functions $\eta_{i}^{\alpha}$ satisfy the bound

$$
\sup _{M}\left(\delta\left|\mathcal{L} \eta_{i}^{\alpha}\right|+\epsilon\left|\nabla \eta_{i}^{\alpha}\right|\right) \leq c
$$

and any $\eta \in K_{0}$ satisfies

$$
\|\mathcal{L} \eta\|_{L^{2}(M)} \leq c \delta^{\frac{n-2}{2}}\|\eta\|_{L^{2}(M)} .
$$

2.3. Conformally Invariant Sobolev Inequality. It is necessary for us to consider approximate solutions constructed from arbitrarily strong dilations, or equivalently, we must allow $\epsilon$ to be arbitrarily small. This fact makes the analysis of (2.1) difficult because the geometry of the manifold $\left(M, g_{1_{k}}\right)$ degenerates as $\epsilon \rightarrow 0$. In particular, the injectivity radius of the neck region $N_{+i}^{(\delta)}$ tends to zero as the strength of the dilation, $G_{+i}$, tends to infinity. This difficulty is overcome by exploiting the fact that

$$
\Omega^{(+\delta)}=N_{-1}^{(\delta)} \cup \Omega \cup N_{+k}^{(\delta)}
$$

is conformally equivalent to a subdomain of $S^{n}$, and has scalar curvature which is bounded independent of $\epsilon$. It is this property of our approximate solutions which allows us to use the following Sobolev inequality.

Lemma 2.3. If $(M, h)$ has bounded scalar curvature, $|R(h)| \leq c_{1}$, and is conformally equivalent to a subdomain of a compact manifold $(N, g)$, with positive scalar curvature, then for any $\phi \in C_{c}^{\infty}(M)$, the inequality

$$
\left(\int_{M} \phi^{\frac{2 n}{n-2}} d v_{h}\right)^{\frac{n-2}{n}} \leq c \int_{M}\left(|\nabla \phi|^{2}+\phi^{2}\right) d v_{h}
$$

holds for $c=c\left(c_{1}, I(g)\right)$, where $I(g)>0$ is the Yamabe invariant of $(N, g)$.

Proof. Recall that the definition of the Yamabe invariant given in (0.4) is equivalent to

$$
I(g)=\inf _{\phi \in C^{\infty}(N)} \frac{-\int \phi L_{g} \phi d v_{g}}{\left(\int \phi^{\frac{2 n}{n-2}} d v_{g}\right)^{\frac{n-2}{n}}}
$$

where $L_{g}$ is the conformal Laplacian taken with respect to $g$ and the integrals are taken over all of $\mathrm{N}$. Let

$$
\Phi: U \rightarrow M
$$


be the conformal diffeomorphism of a subdomain $U$ of $N$ onto $M$, so that

$$
\Phi^{*}(h)=u^{\frac{4}{n-2}} g=\bar{g}
$$

for some $u \in C^{\infty}(U)$. By the conformal invariance of $L_{g}$, we have

$$
L_{\bar{g}}(\psi)=u^{-\frac{n+2}{n-2}} L_{g}(\psi u),
$$

for any $\psi \in C_{c}^{\infty}(U)$. Thus given any $\phi \in C_{c}^{\infty}(M), \psi=\phi \cdot \Phi \in C_{c}^{\infty}(U)$ so we have

$$
I(g) \leq \frac{-\int(\psi u) L_{g}(\psi u) d v_{g}}{\left(\int(\psi u)^{\frac{2 n}{n-2}} d v_{g}\right)^{\frac{n-2}{n}}}=\frac{-\int \psi L_{\bar{g}}(\psi) d v_{\bar{g}}}{\left(\int \psi^{\frac{2 n}{n-2}} d v_{\bar{g}}\right)^{\frac{n-2}{n}}},
$$

where the integrals are taken over $U$. After a change of variables this implies that

$$
\left(\int_{M} \phi^{\frac{2 n}{n-2}} d v_{h}\right)^{\frac{n-2}{n}} \leq \frac{1}{I(g)} \int_{M}\left(|\nabla \phi|^{2}+c(n) R(h) \phi^{2}\right) d v_{h} .
$$

Choosing $c=\max \left(1, c(n) c_{1}\right)$ then completes the proof of Lemma 2.3.

2.4. $L^{2}(M)$ Estimates for $K_{0}^{\perp}$. To prove the existence of a small eigenspace $K$ close to $K_{0}$, we need prove estimates for functions $\eta \in K_{0}^{\perp}$, which indicate that $\mathcal{L}$ is bounded below in $K_{0}^{\perp} \subset L^{2}(M)$, in the operator norm on $L^{2}(M)$. Here $K_{0}^{\perp}$ denotes the orthogonal complement of $K_{0}$ in $L^{2}(M)$. We define the orthogonal projection operators $p_{0}$ and $q_{0}$ by

$$
\begin{array}{ll}
p_{0} & : L^{2}(M) \rightarrow K_{0} \\
q_{0} & : L^{2}(M) \rightarrow K_{0}^{\perp} .
\end{array}
$$

In addition, for $1 \leq i \leq k$, we define $K_{0}^{(i)}$ to be the linear span of $\eta_{i}^{\alpha}$ for $\alpha=1, \ldots, n+1$, and let $p_{0}^{(i)}$ and $q_{0}^{(i)}$ denote the orthogonal projections of $L^{2}(M)$ onto $K_{0}^{(i)}$ and $K_{0}^{(i)^{\perp}}$, respectively. For $2 \leq i \leq k-1$, we define

$$
\widehat{\Omega}_{i}=\Omega_{i-1} \cup \Omega_{i} \cup \Omega_{i+1} .
$$

For the terminal vertices, and the ends $M_{0}$ and $S_{0}^{n}$, defined in the decomposition (1.7) we define

$$
\begin{array}{ll}
\widehat{\Omega}_{1}=M_{0} \cup \Omega_{1} \cup \Omega_{2}, & \widehat{\Omega}_{k}=\Omega_{k-1} \cup \Omega_{k} \cup S_{0}^{n}, \\
\widehat{M}_{0}=M_{0} \cup \Omega_{1}, & \widehat{S}_{0}^{n}=S_{0}^{n} \cup \Omega_{k} .
\end{array}
$$

The following lemma then holds. 
Lemma 2.4. Suppose $\eta \in K_{0}^{(i)^{\perp}} \cap C^{\infty}(M)$, then the estimate

$$
\|\eta\|_{L^{2}\left(\Omega_{i}\right)} \leq\|\mathcal{L} \eta\|_{L^{2}\left(\widehat{\Omega}_{i}\right)}+c(\log (1 / \epsilon))^{\frac{(1-n)}{n}}\|\eta\|_{L^{2}\left(\widehat{\Omega}_{i}\right)}
$$

holds. Moreover, for $\eta \in C^{\infty}(M)$ we have the following estimates on the ends, $M_{0}$ and $S_{0}^{n}$ of $M$,

$$
\begin{aligned}
\|\eta\|_{L^{2}\left(M_{0}\right)} & \leq\|\mathcal{L} \eta\|_{L^{2}\left(\widehat{M}_{0}\right)}+c(\log (1 / \epsilon))^{\frac{(1-n)}{n}}\|\eta\|_{L^{2}\left(\widehat{M}_{0}\right)} \\
\|\eta\|_{L^{2}\left(S_{0}^{n}\right)} & \leq\|\mathcal{L} \eta\|_{L^{2}\left(\widehat{S}_{0}^{n}\right)}+c(\log (1 / \epsilon))^{\frac{(1-n)}{n}}\|\eta\|_{L^{2}\left(\widehat{S}_{0}^{n}\right)}
\end{aligned}
$$

Proof. This is virtually identical to [15, Lemma 3.4], the distinction being that to derive the estimates on the ends we must obtain the inequality

$$
\int_{M}\left(\Psi_{M_{0}} \eta\right)^{2} d v_{1} \leq c\left(\bar{\eta}_{1}\right)^{2}+\int_{M}\left(\left|\nabla\left(\Psi_{M_{0}} \eta\right)\right|^{2}-n\left(\Psi_{M_{0}} \eta\right)^{2}\right) d v_{1}
$$

where $\eta \in C^{\infty}(M)$, and $\Psi_{M_{0}}$ is a cut off function whose support is contained in $M_{0}^{\left(-\delta_{1}\right)}$, and $\bar{\eta}_{1}$ denotes the average of $\Psi_{M_{0}} \eta$ over $(M, g)$. On the asr's this is identical to $[15,(3.6)]$.

From inequality (2.7) the derivation of the estimates in Lemma 2.4 is identical to the argument given in $[15,356-359]$. The inequality for the function $\Psi_{S_{0}^{n}} \eta$ is derived in exactly the same manner. Since $\Psi_{M_{0}} \eta$ is supported in $M_{0}^{\left(-\delta_{1}\right)}$, where $g_{1}$ is isometric to the nondegenerate metric $g$, we may use spectral information on $(M, g)$ instead of $\left(S^{n}, g_{0}\right)$.

We obtain the estimate (2.7) as follows. Since

$$
\bar{\eta}_{1}=\operatorname{Vol}_{g}(M)^{-1} \int_{M} \Psi_{M_{0}} \eta d v_{1}
$$

the variational characterization of $\lambda_{1}$ allows us to conclude that

$$
\lambda_{1} \int_{M}\left(\Psi_{M_{0}} \eta-\bar{\eta}_{1}\right)^{2} d v_{g} \leq \int_{M}\left|\nabla\left(\Psi_{M_{0}} \eta\right)\right|^{2} d v_{g}
$$

Since $g$ is a minimal, nondegenerate solution we have $\lambda_{1}>n$, this then gives us

$$
\int_{M}\left(\Psi_{M_{0}} \eta-\bar{\eta}_{1}\right)^{2} d v_{g} \leq\left(\lambda_{1}-n\right)^{-1} \int_{M}\left(\left|\nabla\left(\Psi_{M_{0}} \eta\right)\right|^{2}-n\left(\Psi_{M_{0}} \eta-\bar{\eta}_{1}\right)^{2}\right) d v_{g}
$$

From this inequality we immediately derive (2.7) for the function $\Psi_{M_{0}} \eta$. The proof then proceeds as in $[15,356-359]$. We refer to there for the remainder of the proof. 
Lemma 2.4 easily gives the following Corollary which is fundamental to the linear analysis of (2.1).

Corollary 2.5. Suppose $\eta \in K_{0}^{\perp} \cap C^{\infty}(M)$. We then have the bound

$$
\|\eta\|_{L^{2}(M)} \leq c\|\mathcal{L} \eta\|_{L^{2}(M)}
$$

provided $\epsilon$ is small enough.

Proof. By applying Lemma 2.4 over each component of the decomposition

$$
M=M_{0} \cup \Omega \cup S_{0}^{n}, \quad \Omega=\cup_{i=1}^{k} \Omega_{i},
$$

we have

$$
\begin{aligned}
\int_{M} \eta^{2} d v_{1} & =\int_{M_{0}} \eta^{2} d v_{1}+\sum_{i=1}^{k} \int_{\Omega_{i}} \eta^{2} d v_{1}+\int_{S_{0}^{n}} \eta^{2} d v_{1} \\
& \leq c \int_{M}(\mathcal{L} \eta)^{2} d v_{1}+c \log (1 / \epsilon)^{\frac{2(1-n)}{n}} \int_{M} \eta^{2} d v_{1} .
\end{aligned}
$$

Choosing $\epsilon$ sufficiently small then completes the proof.

2.5. The Small Eigenspace $K$. If $\phi$ is an eigenfunction of $\mathcal{L}$ with eigenvalue $\lambda$, then $\phi$ is also an eigenfunction for $\Delta_{g_{1}}$ with eigenvalue $\lambda+n$. In particular, there is a one-to-one correspondence between the spectum of $\Delta_{g_{1}}$ on $M$, that is, the set of eigenvalues for $\Delta_{g_{1}}$, and the spectrum of $\mathcal{L}$ on $M$, which we denote by $\sigma(\mathcal{L})$. A consequence of the basic spectral theory for the self adjoint operator $\Delta_{g_{1}}$ on $L^{2}(M)$ (see [2]), is that $\sigma(\mathcal{L})=\left\{\lambda_{i}\right\}_{i=0}^{\infty}$ is a discrete set tending to $+\infty$,

$$
\lambda_{0}=-n<\lambda_{1} \leq \lambda_{2} \leq \cdots
$$

and the corresponding eigenfunctions $\phi_{i} \subset L^{2}(M) \cap C^{\infty}(M)$ (normalized so that $\left\|\phi_{i}\right\|_{L^{2}}=1$ ), form an orthonormal basis for $L^{2}(M)$. It follows that for any $\eta \in L^{2}(M)$ we can write $\eta=\sum_{i=0}^{\infty} a_{i} \phi_{i}$ and $\mathcal{L} \eta=-\sum_{i=0}^{\infty} a_{i} \lambda_{i} \phi_{i}$. When $\eta \in K_{0}$, the inequality $\|\mathcal{L} \eta\|_{L^{2}(M)} \leq c_{K_{0}} \delta^{\frac{n-2}{2}}\|\eta\|_{L^{2}(M)}$ of Proposition 2.2 is then equivalent to

$$
\sum_{i=0}^{\infty} a_{i}^{2}\left(\lambda_{i}^{2}-c_{K_{0}}^{2} \delta^{n-2}\right) \leq 0
$$


The corresponding inequality $\|\eta\|_{L^{2}(M)} \leq c_{K_{0}^{1}}\|\mathcal{L} \eta\|_{L^{2}(M)}$ of Corollary 2.5 for $\eta \in K_{0}^{\perp}$ is equivalent to

$$
\sum_{i=0}^{\infty} a_{i}^{2}\left(1-c_{K_{0}^{\perp}}^{2} \lambda_{i}^{2}\right) \leq 0
$$

We define the subspace $K \subset L^{2}(M)$ of small eigenfunctions by

$$
K=\operatorname{span}\left\{\phi_{i}:\left|\lambda_{i}\right| \leq c_{K_{0}} \delta^{\frac{n-2}{2}}\right\}
$$

If there were an eigenvalue $\lambda_{j}$ such that $\lambda_{j}>c_{K_{0}} \delta^{\frac{n-2}{2}}$ which also satisfied $\lambda_{j}<1 / c_{K_{0}^{\perp}}$ then the corresponding eigenfunction, $\phi_{j}$ would belong to neither $K_{0}$ nor $K_{0}^{\perp}$, since each of the necessary inequalities would be violated. Since $L^{2}(M)=K_{0} \oplus K_{0}^{\perp}$ no such eigenvalue can exist.

The above consequences of Proposition 2.2 and Corollary 2.5, indicate that the closed subspace $K \subset L^{2}(M) \cap C^{\infty}(M)$ is invariant with respect to the operator $\mathcal{L}$, i.e. $\mathcal{L}: K \rightarrow K$, and has the properties

$$
\begin{array}{lll}
\|\mathcal{L} \eta\|_{L^{2}(M)} & \leq c \delta^{\frac{n-2}{2}}\|\eta\|_{L^{2}(M)} & \text { for } \quad \eta \in K \\
\|\eta\|_{L^{2}(M)} & \leq c\|\mathcal{L} \eta\|_{L^{2}(M)} & \text { for } \quad \eta \in K^{\perp} \cap C^{\infty}(M)
\end{array}
$$

Let

$$
\begin{aligned}
& \mathbf{p}: L^{2}(M) \rightarrow K \\
& \mathbf{q}: L^{2}(M) \rightarrow K^{\perp}
\end{aligned}
$$

be the orthogonal projection operators. The following Lemma is analogous to [15, Lemma 3.6], and reflects the extent to which we can use the explicit nature of $K_{0}$ to control the behavior of $K$.

Lemma 2.6. For any $\eta \in L^{2}(M)$,

$$
\left\|\mathbf{p}(\eta)-\mathbf{p}_{0}(\eta)\right\|_{L^{2}(M)}=\left\|\mathbf{q}(\eta)-\mathbf{q}_{0}(\eta)\right\|_{L^{2}(M)} \leq c \delta^{\frac{n-2}{2}}\|\eta\|_{L^{2}(M)}
$$


There is a basis $\phi_{i}^{\alpha}, i=1, \ldots, k, \alpha=1, \ldots, n+1$, for $K$ satisfying for $i \neq j$

$$
\begin{aligned}
\left\|\phi_{i}^{\alpha}-\eta_{i}^{\alpha}\right\|_{L^{2}(M)} & \leq c \delta^{\frac{n-2}{2}} \\
\left\|\phi_{i}^{\alpha}\right\|_{L^{2}\left(M_{0}^{(+\delta)}\right)} & \leq c \delta^{\frac{n-2}{2}} \\
\left\|\phi_{i}^{\alpha}\right\|_{L^{2}\left(\Omega_{j}^{(+\delta)}\right)} & \leq c \delta^{\frac{n-2}{2}} \\
\left\|\phi_{i}^{\alpha}\right\|_{L^{2}\left(S_{0}^{n(+\delta)}\right)} & \leq c \delta^{\frac{n-2}{2}}, \\
\left|\left\langle\phi_{i}^{\alpha}, \phi_{j}^{\beta}\right\rangle_{L^{2}(M)}\right| & \leq c \delta^{\frac{n-2}{2}} .
\end{aligned}
$$

Remark 2.1. In [15, Lemma 3.6], Schoen establishes this result by proving exponential decay in $D(i, j)$, the graph distance between the vertices $i$ and $j$, for the latter two quantities. This more difficult result is necessary, in part, due to the exponential volume growth that Schoen's approximate solutions may exhibit. Since our approximate solutions are compact and have volumes which may be estimated as in Theorem 1.3, the estimates which we prove here are sufficient. We refer to [15] for the proof of the $L^{2}(M)$ estimates on the difference of the projection operators, and here establish the estimates on the basis $\left\{\phi_{i}^{\alpha}\right\}$ for $K$.

Proof. We first define the basis element $\phi_{i}^{\alpha}$ to be the component of the orthogonal projection of $\eta_{i}^{\alpha}$ into $K$ which arises solely from $\eta_{i}^{\alpha}$. Explicitly, for each $i=1, \ldots, k$ let $\hat{K}_{i}$ denote the linear span of $\mathbf{p}\left(\eta_{j}^{\alpha}\right), j \neq i, \alpha=1, \ldots, n+1$. We define $\phi_{i}^{\alpha}$ by

$$
\phi_{i}^{\alpha}=\mathbf{p}\left(\eta_{i}^{\alpha}\right)-\hat{\mathbf{p}}^{(i)}\left(\eta_{i}^{\alpha}\right),
$$

where $\hat{\mathbf{p}}^{(i)}: L^{2}(M) \rightarrow \hat{K}_{i}$ is the orthogonal projection operator. If $\phi=$ $\sum_{j \neq i, \beta} a_{j}^{\beta} \mathbf{p}\left(\eta_{j}^{\beta}\right) \in \hat{K}_{i}$ with $\|\phi\|_{L^{2}(M)}=1$, then $\phi=\mathbf{p}(\eta)$, where $\eta=\sum_{j \neq i, \beta} a_{j}^{\beta} \eta_{j}^{\beta}$ $\in K_{0}$. We then have

$$
\|\phi-\eta\|_{L^{2}(M)} \leq c \delta^{\frac{n-2}{2}}\|\eta\|_{L^{2}(M)}
$$

and hence

$$
\|\eta\|_{L^{2}(M)} \leq c .
$$

Since $\eta=\mathbf{p}_{0}(\eta)$ we may write

$$
\left\langle\eta_{i}^{\alpha}, \phi\right\rangle_{L^{2}(M)}=\left\langle\eta_{i}^{\alpha}, \eta\right\rangle_{L^{2}(M)}+\left\langle\eta_{i}^{\alpha}, \mathbf{p}(\eta)-\mathbf{p}_{0}(\eta)\right\rangle_{L^{2}(M)} .
$$


Therefore, we have

$$
\left|\left\langle\eta_{i}^{\alpha}, \phi\right\rangle_{L^{2}(M)}\right| \leq\left|\sum_{\beta=1}^{n+1}\left(a_{i-1}^{\beta}\left\langle\eta_{i^{\prime}}^{\alpha}, \eta_{i 1}^{\beta}\right\rangle_{L^{2}(M)}+a_{i+1}^{\beta}\left\langle\eta_{i}^{\alpha}, \eta_{i+1}^{\beta}\right\rangle_{L^{2}(M)}\right)\right|+c \delta^{\frac{n-2}{2}}
$$

By the definition of the functions $\eta_{i}^{\alpha}$ we immediately have

$$
\begin{aligned}
\left|\sum_{\beta=1}^{n+1}\left(a_{i-1}^{\beta}\left\langle\eta_{i}^{\alpha}, \eta_{i 1}^{\beta}\right\rangle_{L^{2}(M)}+a_{i+1}^{\beta}\left\langle\eta_{i}^{\alpha}, \eta_{i+1}^{\beta}\right\rangle_{L^{2}(M)}\right)\right| & \leq c \delta^{n} \sum_{\beta=1}^{n+1}\left(\left|a_{i+1}^{\beta}\right|+\left|a_{i-1}^{\beta}\right|\right) \\
& \leq c \delta^{n} \sum_{\beta=1}^{n+1}\left(\left|a_{i+1}^{\beta}\right|^{2}+\left|a_{i-1}^{\beta}\right|^{2}\right)^{\frac{1}{2}}
\end{aligned}
$$

On the other hand, since

$$
\begin{aligned}
\sum_{\beta, \gamma, j \neq i}\left|a_{j}^{\beta}\right|\left(\left|a_{j-1}^{\gamma}\right|+\left|a_{j+1}^{\gamma}\right|\right) & \leq c \sum_{\beta, j}\left(a_{j}^{\beta}\right)^{2}+\sum_{\beta, \gamma, j}\left(\left(a_{j-1}^{\gamma}\right)^{2}+\left(a_{j+1}^{\gamma}\right)^{2}\right) \\
& \leq c \sum_{\beta, j}\left(a_{j}^{\beta}\right)^{2}
\end{aligned}
$$

we can estimate

$$
\begin{aligned}
\langle\eta, \eta\rangle_{L^{2}(M)} & =\sum a_{j}^{\beta}\left\langle\eta_{j}^{\beta}, \sum a_{l}^{\gamma} \eta_{l}^{\gamma}\right\rangle \\
& \geq c \sum\left(a_{j}^{\beta}\right)^{2}-c \delta^{n} \sum_{\beta, \gamma, j \neq i}\left|a_{j}^{\beta}\right|\left(\left|a_{j-1}^{\gamma}\right|+\left|a_{j+1}^{\gamma}\right|\right) \\
& \geq c \sum\left(a_{j}^{\beta}\right)^{2} .
\end{aligned}
$$

Thus using this estimate above and the $L^{2}(M)$ bound on $\eta$, we have

$$
\left|\left\langle\eta_{\alpha}^{i}, \phi\right\rangle\right| \leq c \delta^{\frac{n-2}{2}}
$$

If we let

$$
\phi=\frac{\hat{\mathbf{p}}^{(i)}\left(\eta_{i}^{\alpha}\right)}{\left\|\hat{\mathbf{p}}^{(i)}\left(\eta_{i}^{\alpha}\right)\right\|_{L^{2}(M)}}
$$

then it follows that

$$
\left\|\hat{\mathbf{p}}^{(i)}\left(\eta_{i}^{\alpha}\right)\right\|_{L^{2}(M)} \leq c \delta^{\frac{n-2}{2}}
$$

Thus,

$$
\begin{aligned}
\left\|\phi_{i}^{\alpha}-\eta_{i}^{\alpha}\right\|_{L^{2}(M)} & \leq\left\|\mathbf{p}\left(\eta_{i}^{\alpha}\right)-\mathbf{p}_{0}\left(\eta_{i}^{\alpha}\right)\right\|_{L^{2}(M)}+\left\|\hat{\mathbf{p}}^{(i)}\left(\eta_{i}^{\alpha}\right)\right\|_{L^{2}(M)} \\
& \leq c \delta^{\frac{n-2}{2}}
\end{aligned}
$$


This establishes the first property of the functions $\phi_{i}^{\alpha}$. The other properties follow easily from this. The $L^{2}(M)$ estimates for $\phi_{i}^{\alpha}$ on each asr and on the ends $M_{0}$ and $S_{0}^{n}$ are a direct consequence, as follows

$$
\begin{aligned}
\left\|\phi_{i}^{\alpha}\right\|_{L^{2}\left(\Omega_{j}^{(+\delta)}\right)} & \leq\left\|\phi_{i}^{\alpha}-\eta_{i}^{\alpha}\right\|_{L^{2}(M)}+\left\|\eta_{i}^{\alpha}\right\|_{L^{2}\left(\Omega_{j}^{(+\delta)}\right)} \\
& \leq c \delta^{\frac{n-2}{2}}+c \delta^{n} \\
& \leq c \delta^{\frac{n-2}{2}} .
\end{aligned}
$$

The corresponding estimates on the ends $M_{0}$ and $S_{0}^{n}$ follow in exactly the same way. The final estimate is then derived from this. First note that since $\hat{\mathbf{p}}^{(j)}\left(\eta_{j}^{\beta}\right)$ is orthogonal to $\phi_{i}^{\alpha}$, we have

$$
\begin{aligned}
\left\langle\phi_{i}^{\alpha}, \phi_{j}^{\beta}\right\rangle_{L^{2}(M)} & =\left\langle\phi_{i}^{\alpha}, \mathbf{p}\left(\eta_{j}^{\beta}\right)\right\rangle_{L^{2}(M)} \\
& =\left\langle\phi_{i}^{\alpha}, \eta_{j}^{\beta}\right\rangle_{L^{2}(M)} .
\end{aligned}
$$

Thus since $\eta_{j}^{\beta}$ has its support contained within $\Omega_{j}^{(+\delta)}$, we have

$$
\begin{aligned}
\left|\left\langle\phi_{i}^{\alpha}, \phi_{j}^{\beta}\right\rangle_{L^{2}(M)}\right| & =\left|\left\langle\phi_{i}^{\alpha}, \eta_{j}^{\beta}\right\rangle_{L^{2}(M)}\right| \\
& \leq\left\|\eta_{j}^{\beta}\right\|_{L^{2}\left(\Omega_{j}^{(+\delta)}\right)}\left\|\phi_{i}^{\alpha}\right\|_{L^{2}\left(\Omega_{j}^{(+\delta)}\right)} \\
& \leq c \delta^{\frac{n-2}{2}} .
\end{aligned}
$$

This completes the proof of Lemma (2.6).

2.6. Pointwise Estimates for the Solution of the Linear Problem. To study the solution of the linear equation, $\mathcal{L} \eta=f$, on the orthogonal complement of the small eigenspace $K$, we first derive pointwise estimates for $\mathcal{L}$. The main task is to derive an $\epsilon$ - independent $C^{0}$ estimate. Recall that the condition for our conformal structure to be admissible ensures that the parameters $\epsilon_{ \pm i}=2 \lambda_{ \pm i}^{\frac{1}{2}}$ are comparable to $\epsilon$ as in (1.6). As $\epsilon$ tends to zero the geometry of our solutions degenerate. In light of this, we make a choice of the $C^{r, \alpha}$ norm on $M$ which will encode the $\epsilon$ dependence of our estimates. As in [15] we first make a choice of the $C^{r, \alpha}$ norm on $\left(S^{n}, g_{0}\right)$. For a domain $\mathcal{O} \subset S^{n}$, and $\eta \in C^{r, \alpha}(\mathcal{O})$, we let

$$
\|\eta\|_{r, \alpha, \mathcal{O}}=\sum_{s=0}^{r} \epsilon^{s} \sup _{\mathcal{O}}\left|\partial^{(s)} \eta\right|+\epsilon^{r+\alpha}\left\|\partial^{(r)} \eta\right\|_{(\alpha), \mathcal{O}}
$$


where $\left|\partial^{(s)} \eta\right|$ is the length with respect to $g_{0}$ of covariant derivatives of $\eta$ of order $s$. The quantity $\left\|\partial^{(r)} \eta\right\|_{(\alpha), \mathcal{O}}$ is the Hölder exponent of $r$-th derivatives of $\eta$, which we define by using a fixed coordinate covering of $S^{n}$. Similarly we make a choice of $C^{r, \alpha}$ norm on domains in $(M, g)$ and $\left(S^{n}, \bar{g}\right)$, defined with respect to the fixed background metrics $g$ and $\bar{g}$. For any $\eta \in C^{r, \alpha}(M)$ we define

$$
\|\eta\|_{r, \alpha}=\max _{1 \leq i \leq k}\left\{\left\|\eta \cdot F_{i}^{-1}\right\|_{r, \alpha, \mathcal{O}_{i}},\|\eta\|_{r, \alpha, M_{0}},\|\eta\|_{r, \alpha, S_{0}^{n}}\right\},
$$

where $\mathcal{O}_{i}=F_{i}\left(\Omega_{i}\right)$ and $M_{0}$ and $S_{0}^{n}$ are the ends of our approximate solution given by the decomposition (1.7). The following Theorem is analogous to [15, Theorem 3.9] and establishes the basic pointwise estimate for $\eta \in K$.

Theorem 2.7. For any non-negative integer $r$ and any $\alpha \in(0,1)$

$$
\|\eta\|_{r, \alpha} \leq c\|\eta\|_{L^{2}(M)}
$$

for every $\eta \in K$, where $c=c(r, \alpha)$ is independent of $\epsilon$. Thus the inclusion $K \subset C^{r, \alpha}(M)$ has bounded norm.

Proof. Due to its central role we sketch the proof of Theorem 2.7 here, referring to [15, Theorem 3.9] for more details.

Note that $K \subset C^{\infty} \cap L^{2}(M)$, follows immediately from elliptic regularity since $K$ is constructed from eigenfunctions of $\Delta_{g_{1}}$ on $M$. We first derive the supremum estimate for $\eta \in K$. For any point $p \in M, B_{\delta / 2}(p)$ is contained in either $M_{0}^{(\delta)}, S_{0}^{n^{(\delta)}}$ or $\Omega_{i}^{(\delta)}$ for some $i$, where $\delta>c \epsilon^{1-\frac{2}{n}}$. Since $\delta \rightarrow 0$ as $\epsilon \rightarrow 0$, this does not allow us to apply the Sobolev inequality, Lemma 0.4, in balls whose radius is uniformly bounded below independent of $\epsilon$. We avoid this difficulty by noting that both $(M, g)$ and $\left(S^{n}, \bar{g}_{0}\right)$ are locally conformally flat in a ball of radius $r=\min \left(r(g), r\left(\bar{g}_{0}\right)\right)$ about the point at which $\Omega=\cup_{i=1}^{k} \Omega_{i}$ is attached. Thus we may choose some $\rho<r / 2$ independent of $\epsilon$, so that $B_{\rho}(p)$ is contained in either $\Omega^{(r)}, M_{0}^{(r)}$ or $S_{0}^{n^{(r)}}$. These domains are conformally equivalent to a domain in $\left(S^{n}, g_{0}\right),(M, g)$ or $\left(S^{n}, \bar{g}_{0}\right)$, respectively. Thus we may apply the Sobolev inequality in $B_{\rho}(p)$, for any $p \in M$. This allows us to use the De Giorgi-Nash-Moser theory (see [4, Theorem 8.17]) to locally 
estimate the supremum norm of $\eta$

$$
\sup _{B_{\rho / 2}(p)}|\eta| \leq c\|\eta\|_{L^{2}\left(B_{\rho}(p)\right)}+c\|\mathcal{L} \eta\|_{L^{q}\left(B_{\rho}(p)\right)}
$$

where $q>\frac{n}{2}$, and $c$ depends on $q, \rho$ and the Sobolev constant. Since we may choose $\rho$ to be bounded from below, say $\rho>r / 4$ and we may bound the Sobolev constant in terms of the maximum of the Yamabe constants of $\left(S^{n}, g_{0}\right),(M, g)$ and $\left(S^{n}, \bar{g}_{0}\right)$, we regard $c$ as depending on $q$ alone. To estimate the $L^{q}(M)$ norms of $\mathcal{L} \eta$, we again make repeated use of the Sobolev inequality. Let $\zeta \in C^{\infty}$ have support contained in either $M_{0}^{(r)}, S_{0}^{n^{(r)}}$ or $\Omega^{(r)}$ and let $\beta \geq 2$ and any $\eta_{0} \in C^{\infty}(M)$ be given. After integrating by parts, we have

$$
\begin{aligned}
-\int_{M} \zeta^{2} \eta_{0}^{\beta-1} \mathcal{L} \eta_{0} d v_{1}= & \int_{M} \zeta^{2}\left((\beta-1) \eta_{0}^{\beta-2}\left|\nabla \eta_{0}\right|^{2}-n \eta_{0}^{\beta}\right) d v_{1} \\
& +2 \int_{M}\left(\zeta \eta_{0}^{\beta-1} \nabla \zeta \cdot \nabla \eta_{0}\right) d v_{1}
\end{aligned}
$$

The supremum estimate is then established by applying the Sobolev inequality to $\phi=\zeta\left|\eta_{0}\right|^{\beta / 2}$ with $\eta_{0}=\mathcal{L} \eta$ and iterating a finite number of times to obtain

$$
\|\mathcal{L} \eta\|_{L^{\beta_{s}}\left(B_{\rho}(p)\right)} \leq c\|\eta\|_{L^{2}(M)} .
$$

where $c$ depends on $s, \beta_{s}=2 \kappa^{s}$, and $\kappa=\frac{n}{n-2}$. Combining this with the De Giorgi-Nash-Moser estimate (2.10) (with $q=\beta^{s}$ ) and summing over a fixed covering of $M$ by balls of radius $\rho$ we get the supremum estimate

$$
\sup _{M}|\eta| \leq c\|\eta\|_{L^{2}(M)}
$$

We now use the interior Schauder estimates (see [4]) to derive the higher derivative estimates for $\eta$. About each point $p \in M$ the metric $g_{1}$ is uniformly equivalent to one of the background metrics $g_{0}$ and $\bar{g}_{0}$ on $S^{n}$, or $g$ on $M$, in a ball of radius proportional to $\epsilon$, the proportionality constant being $c_{1}=\beta$ from (1.6). This follows immediately from the bubble construction $\S 2.4$ and the fact that $g_{1}$ is constructed from an admissible conformal structure. If $p \in \bar{\Omega}_{i}$ then we may compare the metric $\hat{g}_{1}=\left(F_{i}^{-1}\right)^{*} g_{1}$ to the spherical metric $g_{0}$ in a ball centered at $q=F_{i}(p)$. If $x^{1}, \ldots, x^{n}$ denote normal coordinates for $g_{0}$ centered 
at $q$, then for $|x| \leq \hat{\epsilon} \equiv c_{1}^{-1}, \hat{g}_{1}=\sum h_{\alpha \beta} d x^{\alpha} d x^{\beta}$, where

$$
\begin{gathered}
c^{-1} \sum \xi_{\alpha}^{2} \leq \sum h_{\alpha \beta} \xi_{\alpha} \xi_{\beta} \leq c \sum \xi_{\alpha}^{2}, \\
\epsilon^{r}\left|\partial^{(r)} h_{\alpha \beta}\right| \leq c,
\end{gathered}
$$

$\left|\partial^{(r)} h_{\alpha \beta}\right|$ denoting the absolute value of partial derivatives of order $r$. A similar estimate holds for $p$ in either $\bar{M}_{0}$ or $\overline{S_{0}^{n}}$. For example, if $p \in \bar{M}_{0} \subset M_{0}^{(-\delta)} \cup N_{-1}^{(\delta)}$ then we distinguish between which region $p$ lies in as follows. If $p \in M_{0}^{(-\delta)}$ then we use the identity map and note that $g_{1}$ is isometric to $g$ in a ball of radius $\hat{\epsilon}$ about $p$. If $p \in N_{-1}^{(\delta)}$ then the metric $\hat{g}_{1}=\left(F_{0}^{-1} \cdot \Phi\right)^{*} g_{1}$ is uniformly equivalent to the background metric $g$ on $M$ in a ball of radius $\hat{\epsilon}$ about $q=\Phi^{-1} \cdot F_{0}(p)$, and if we let $x^{1}, \ldots, x^{n}$ denote normal coordinates for $g$ then the above inequalities are also valid in this ball.

If $p \in \bar{\Omega}$, say $p \in \bar{\Omega}_{i}$ then the Schauder estimate implies that

$$
\left\|\eta \cdot F_{i}^{-1}\right\|_{2, \alpha, D_{\varepsilon / 2}} \leq c\left(\epsilon^{2}\left\|\mathcal{L} \eta \cdot F_{i}^{-1}\right\|_{0, \alpha, D_{\varepsilon}}+\sup _{D_{\tilde{\varepsilon}}}\left|\eta \cdot F_{i}^{-1}\right|\right),
$$

where $D_{\hat{\epsilon}}=\{x:|x| \leq \hat{\epsilon}\}$ with respect to normal coordinates and the norms are the $\epsilon$ weighted norms defined above. A similar estimate holds for $p \in \bar{M}_{0}$ and $p \in \overline{S_{0}^{n}}$. Because of the $\epsilon$ weighting of the norms and the uniform equivalence of the metric $g_{1}$ to one of the three fixed metrics $g_{0}, g$ or $\bar{g}_{0}$ in balls of radius proportional to $\epsilon$, we have

$$
\left\|\eta \cdot F_{i}^{-1}\right\|_{r, \alpha, \mathcal{O}_{i}} \leq c \sup _{q_{0} \in \mathcal{O}_{i}}\left\|\eta \cdot F_{i}^{-1}\right\|_{r, \alpha, B_{\tilde{\varepsilon}}\left(q_{0}\right)},
$$

where $c$ depends on $c_{1}$. Note that this estimate also holds on $M_{0}$ and $S_{0}^{n}$. The $C^{2, \alpha}$ estimate now follows by applying the fundamental theorem of calculus and standard estimates for the Poisson equation. We refer to [15, Theorem 3.9] for the remainder of the proof of Theorem 2.7.

We now prove the main theorem concerning solvability of the linear equation $\mathcal{L} \eta=f$.

Theorem 2.8. (i) Suppose $\eta \in C^{r+2, \alpha}(M) \cap K^{\perp}$, where $r$ is a non- negative integer and $\alpha \in(0,1)$. We then have $\mathcal{L} \eta \in C^{r, \alpha}(M) \cap K^{\perp}$, and

$$
\|\eta\|_{r+2, \alpha} \leq c\|\mathcal{L} \eta\|_{r, \alpha}
$$


where $c=c(r, \alpha)$.

(ii) Given $f \in C^{r, \alpha}(M) \cap K^{\perp}$, there is a unique $\eta \in C^{r+2, \alpha}(M) \cap K^{\perp}$ satisfying $\mathcal{L} \eta=f$.

Proof. To prove part (i), let $\eta \in C^{r+2, \alpha}(M) \cap K^{\perp}$. By applying the Schauder estimate as in the previous proof we get

$$
\|\eta\|_{r+2, \alpha} \leq c\left(\epsilon^{2}\|\mathcal{L} \eta\|_{r, \alpha}+\|\eta\|_{C^{\circ}(M)}\right)
$$

where $c$ depends on $c_{1}$. The De Giorgi-Nash-Moser estimate gives us

$$
\|\eta\|_{C^{0}(M)} \leq c\|\eta\|_{L^{2}(M)}+c\|\mathcal{L} \eta\|_{C^{0}(M)}
$$

Since $\eta \in K^{\perp}$ and the volume of $\left(M, g_{1}\right)$ can be estimated by a fixed constant, independent of $\epsilon$, we get

$$
\|\eta\|_{L^{2}(M)} \leq c\|\mathcal{L} \eta\|_{L^{2}(M)} \leq c\|\mathcal{L} \eta\|_{C^{0}(M)}
$$

Therefore

$$
\begin{aligned}
\|\eta\|_{r+2, \alpha} & \leq c\left(\epsilon\|\mathcal{L} \eta\|_{r, \alpha}+c\|\mathcal{L} \eta\|_{C^{\circ}(M)}\right) \\
& \leq c\|\mathcal{L} \eta\|_{r, \alpha}
\end{aligned}
$$

as claimed.

To establish the second claim we note that since $f \in K^{\perp}$, there exists a unique $\eta \in K^{\perp}$ with $\mathcal{L} \eta=f$, namely the function $\eta=\mathcal{L}^{-1} f$. By part (i) of our theorem, since $f \in C^{r, \alpha}(M)$ we have

$$
\|\eta\|_{r+2, \alpha} \leq c\|f\|_{r, \alpha}
$$

Thus $\eta \in C^{r+2, \alpha}(M)$ This completes the proof of Theorem 2.8.

\section{The Projected Problem}

We define the projected problem as

$$
\mathcal{L} \eta=\mathbf{q}(F(x, \eta))
$$

where $\mathbf{q}: L^{2}(M) \rightarrow K^{\perp}$ is the orthogonal projection operator discussed in Lemma 2.6. The linear estimates of section 3 combined with a contraction mapping argument allows us to prove that (3.1) has a unique small solution 
$\eta \in K^{\perp}$. The Theorem is nearly identical to [15, Proposition 4.1], and we refer there for its proof.

Theorem 3.1. There exists a $\delta>0$ depending only on $n, \beta$ such that (3.1) has a unique solution $\eta \in C^{2, \alpha}(M) \cap K^{\perp}$ satisfying $\|\eta\|_{C^{\circ}(M)} \leq \delta$.

We need more precise $C^{0}(M)$ estimates on both the solution $\eta$ to the projected problem (3.1) and on the scalar curvature $R$, of the resulting metric $g=(1+\eta)^{\frac{4}{n-2}} g_{1}$. These can be easily derived from the proof of Theorem 3.1. In $[15]$ it is shown that

$$
\|\eta\|_{C^{0}(M)} \leq c\left(\epsilon^{\frac{n-2}{2}}+\epsilon^{\frac{n-2}{q}}\right)
$$

for any $q>\frac{n}{2}$, and $c=c(q)$.

The scalar curvature $R$ of the metric $g=(1+\eta)^{\frac{4}{n-2}} g_{1}$ is given by

$$
R=-\frac{4(n-1)}{n-2}(1+\eta)^{-\frac{n+2}{n-2}}\left(\Delta_{g_{1}}(1+\eta) \frac{n-2}{4(n-1)} R_{1}(1+\eta)\right) .
$$

Therefore by using the definitions of $F(x, \eta)$ and $Q(\eta)$ in $(2.2)$, we see that

$$
R-n(n-1)=-\frac{4(n-1)}{n-2}(1+\eta)^{-\frac{n+2}{n-2}}[\mathcal{L} \eta-F(x, \eta)]
$$

and hence since $\mathcal{L} \eta=\mathbf{q}(F(x, \eta))$, this implies that

$$
R-n(n-1)=\frac{4(n-1)}{n-2}(1+\eta)^{-\frac{n+2}{n-2}} \mathbf{p}(F(x, \eta)) .
$$

Thus by either $(3.2)$ or simply $\|\eta\|_{C^{\circ}(M)} \leq 1$, and Theorem 2.7 we have

$$
\begin{aligned}
\|R-n(n-1)\|_{C^{0}(M)} & \leq c\|\mathbf{p}(F(x, \eta))\|_{C^{0}(M)} \\
& \leq c\|F(x, \eta)\|_{L^{2}(M)} \\
& \leq c\left\|R_{1}-n(n-1)\right\|_{L^{2}(M)}
\end{aligned}
$$

Hence we conclude that

$$
\|R-n(n-1)\|_{C^{0}(M)} \leq c \epsilon^{\frac{n-2}{2}} .
$$

The solution $g=(1+\eta)^{\frac{4}{n-2}} g_{1}$, to the projected problem (3.1) satisfies the energy estimate of Theorem (0.1). This follows immediately from the volume 
estimate (1.8) on the approximate solution and the $C^{0}(M)$ estimate (3.2) on $\eta$. Since

$$
\operatorname{Vol}_{g}(M)=\int_{M}(1+\eta)^{\frac{2 n}{n-2}} d v_{g_{1}}
$$

we have, for any $q>\frac{n}{2}$,

$$
\begin{aligned}
\left|\operatorname{Vol}_{g}(M)-\operatorname{Vol}_{g_{1}}(M)\right| & \leq c\|\eta\|_{C^{\circ}(M)} \operatorname{Vol}_{g_{1}}(M) \\
& \leq c\left(\epsilon^{\frac{n-2}{2}}+\epsilon^{\frac{n-2}{q}}\right) \operatorname{Vol}_{g_{1}}(M) .
\end{aligned}
$$

Therefore by the triangle inequality and (1.8) we obtain

$$
\left|\operatorname{Vol}_{g}(M)-\left(\operatorname{Vol}_{g_{1}}(M)+(k+1) \omega_{n}\right)\right| \leq c\left(\epsilon^{\frac{n-2}{2}}+\epsilon^{\frac{n-2}{q}}\right) .
$$

\section{The Generalized Pohozaev Identity}

4.1. The Identity. The solution $g=(1+\eta)^{\frac{4}{n-2}} g_{1}$ to the projected problem (3.1), will be an exact solution provided that the function $F(x, \eta)$ lies orthogonal to the small eigenspace $K$, i.e.

$$
\mathbf{q}(F(x, \eta))=F(x, \eta) .
$$

This will not be true in general. It will be necessary to perturb each of the admissible conformal structures $\sigma_{1}, \ldots, \sigma_{N}$ individually, to produce a new admissible $\mathrm{N}$ structure $\sigma^{\prime}(N)$ so that (4.1) holds for each of the new admissible conformal structures $\sigma_{1}^{\prime}, \ldots, \sigma_{N}^{\prime}$. Fortunately we can exhibit an identity which will both provide necessary and sufficient conditions for (4.1) to be satisfied by a given admissible conformal structure and will provide the means to insure that we can find deformations of certain $\sigma(N)$, to a new conformal $N$ structure $\sigma^{\prime}(N)$ which will satisfy these conditions.

The generalized Pohozaev identity is given by the following proposition.

Proposition 4.1. Let $(N, g)$ be a compact Riemannian manifold of dimension $n$ with smooth boundary $\partial N$. Let $R$ denote the scalar curvature of $N$, and suppose that $X$ is a conformal Killing vector field on $N$. We than have the identity

$$
\int_{N}\left(\mathcal{L}_{X} R\right) d v=\frac{2 n}{n-2} \int_{\partial N}\left(R i c_{g}-n^{-1} R g\right)(X, \nu) d \sigma
$$


where $\operatorname{Ric}_{g}(\cdot, \cdot)$ is the Ricci tensor for $(N, g), \mathcal{L}_{X}$ denotes the Lie derivative, $\nu$ denotes the outward unit normal vector to $\partial N$, and $d v, d \sigma$ are volume and surface measure (with respect to $g$ ), respectively.

We refer to [15] for the proof of (4.1). This identity provides an obvious necessary condition for the metric $g$ to have constant scalar curvature $R$, namely the vanishing of the boundary integrals

$$
\int_{\partial \Omega_{i}}\left(R i c_{g}-n^{-1} R g\right)(X, \nu) d \sigma
$$

for all conformal Killing fields $X$ on $\Omega_{i}$. There is a natural class of Conformal killing fields on $\Omega_{i}$ for which we can test this necessary condition. Any vector $\mathbf{w} \in \mathbb{R}^{n+1}$ gives a conformal Killing vector field $W$ on $S^{n}$ by

$$
W(q)=\mathbf{w}-(\mathbf{w} \cdot q) q .
$$

$W$ is the tangential projection of $\mathbf{w}$ onto $S^{n}$. These vector fields are gradient vector fields on $S^{n}$, i.e. they arise as the gradient of a globally defined function on $S^{n}$,

$$
W=\operatorname{grad}_{g_{0}}\left(l_{\mathbf{w}}\right), \quad l_{\mathbf{w}}(q)=\mathbf{w} \cdot q .
$$

We get a conformal Killing field $X$ on $\Omega_{i}$ by defining

$$
X=d F_{i}^{-1}(W) .
$$

Since $F_{i}$ is a conformal diffeomorphism from $\left(\Omega_{i}, g_{1}\right)$ to $\left(\mathcal{O}_{i}, g_{0}\right), X$ is conformal Killing for $g=(1+\eta)^{\frac{4}{n^{2}}} g_{0}$. For $\alpha=1, \ldots, n+1$ we let $X_{\alpha}$ denote the vector field arising from $\mathbf{w}_{\alpha}$, where $\mathbf{w}_{1}, \ldots, \mathbf{w}_{n+1}$ is an orthonormal basis for $\mathbb{R}^{n+1}$.

4.2. Balanced Admissible Conformal Structures. In [15], Schoen computes the boundary integrals (4.3) for the approximate solutions $g_{1}$ constructed in section 2. Recall that $g_{1}$ is scalar flat in a small neighborhood of $\partial \Omega_{i}$. The results of this computation are given by the following Proposition (see [15, Prop. 1.5]).

Proposition 4.2. Let $X$ be the conformal Killing field on $\Omega_{i}$, arising from a vector $\mathbf{w} \in \mathbb{R}^{n+1}$. We have the formula

$$
\int_{\partial \Omega_{i}} \operatorname{Ric}_{g_{1}}(X, \nu) d \sigma_{1}=-2(n-1)(n-2) \omega_{n-1} \mathbf{w} \cdot C_{i},
$$


where $\omega_{n-1}$ is the volume of $S^{n-1}$, and $C_{i} \in \mathbb{R}^{n+1}$ is given by

$$
C_{i}=\epsilon_{-i}^{n-2} q_{-i}+\epsilon_{+i}^{n-2} q_{+i} .
$$

If $X=d F_{i}^{-1}\left(X_{0}\right)$ for a rotation vector field $X_{0}$ on $\left(S^{n}, g_{0}\right)$, then the boundary integral on each component of $\partial \Omega_{i}$ vanishes.

The vector $C_{i}$ is regarded as a "center of mass" attached to each vertex i. $C_{i}$ vanishes if and only if the two dilations $G_{-i}$ and $G_{+i}$ have the same strength and have sources $q_{-i}$ and $q_{+i}$ which are antipodal points on $S^{n}$. An admissible conformal structure whose approximate solution satisfies $C_{i}=0$ for each $i$, will be called a balanced admissible conformal structure. We note that the admissible $\mathrm{N}$ structure, $\sigma(N)$ constructed in $\S 2.6$ consists of balanced admissible conformal structures.

We use the Generalized Pohozaev identity (4.1) in two ways. We first show that it provides a sufficient criteria for determining when the solution to the projected problem is actually an exact solution.

4.3. A Sufficient Criterion for Solutions. If we assume that our approximate solution $g_{1}$ arises from a balanced admissible conformal structure then the vanishing of the boundary integrals (4.3) for those vector fields $X$ arising from a vector $\mathbf{w} \in \mathbb{R}^{n+1}$ is actually sufficient to guarantee that $\mathbf{p}(F(x, \eta))=0$. This result is identical to [15, Prop. 4.4], which we state here for reference.

Proposition 4.3. Suppose $g=(1+\eta)^{\frac{4}{n-2}} g_{1}$ is the metric constructed in Theorem 3.1. Suppose that, for every vertex $i$,

$$
\int_{\partial \Omega_{i}}\left(R i c_{g}-n^{-1} R g\right)(X, \nu) d \sigma_{g}=0
$$

for any conformal vector field $X$ associated with a vector $\mathbf{w} \in \mathbb{R}^{n+1}$. Then $\eta$ is a solution of (2.1); that is, $g$ has scalar curvature identically $n(n-1)$.

Remark 4.1. The idea of the proof is to use the Generalized Pohozaev identity (4.1) to rewrite the vanishing of the boundary integrals above as the vanishing of the integral of a Lie derivative of the scalar curvature, $R(g)$, over each $\Omega_{i}$. One then uses the equation for $R$ and the estimates on $\eta$ found in section 4 to show that this implies that $\mathbf{p}(F(x, \eta))=0$, i.e. $F(x, \eta) \in K^{\perp}$. The Generalized Pohozaev identity thus measures the extent to which $F(x, \eta)$ lies 
orthogonal to $K$. In the last step of the proof Schoen employs the exponential decay estimates for the basis $\left\{\phi_{i}^{\alpha}\right\}$ of $K$, since our solutions are compact and have controlled volume, the estimates we've established in Lemma 2.6 are sufficient here. Thus, if the metric $g=(1+\eta)^{\frac{4}{n-2}} g_{1}$ satisfies the hypothesis of Proposition 4.3, then $\eta$ is actually a solution to (2.1). We refer to [15] for the details of the proof of Proposition 4.3.

In the next section we use the Generalized Pohozaev identity to assign values to deformation parameters which are used to perturb an initial balanced admissible conformal structure to a nearby conformal structure for which the hypothesis of Proposition 4.3 are satisfied.

\section{Deformations of Approximate Solutions}

5.1. Admissible Conformal Structures Revisited. Recall that our admissible conformal $N$ structures, $\sigma(N)$, consist of an assignment of conformal maps to the vertices of each of the $N$ chains, $\mathcal{T}_{1}, \ldots, \mathcal{T}_{N}$, so that the corresponding approximate solutions $g_{1_{1}}, \ldots, g_{1_{N}}$ all lie within a fixed conformal class. In $\S 2.6$ we constructed a conformal $N$ structure, $\sigma(N)$, which had the additional property that each of the admissible conformal structures, $\sigma_{k}$, was balanced, in the sense of $\S 4.2$. We need to consider deformations of $\sigma(N)$, which will consist of deforming each approximate conformal structure, $\sigma_{k}$, separately and then showing that the necessary deformations may be done without changing the conformal class of the corresponding approximate solution metric. The deformations which we consider in this section are exactly the same as those used in [15], however all the approximate solutions considered there were in the conformal class $\left[g_{0}\right]$, of the round metric $g_{0}$ on $S^{n}$, hence the adjustments which we make to the approximate solutions of the new conformal structures to insure that they remain in a fixed conformal class, were unnecessary there. Much of the material in this section can be found in [15], we include it nonetheless, for the sake of completeness. We deform the approximate conformal structures by considering deformations of the conformal maps $F_{i}$, which make up $\sigma_{k}$. Our aim in considering deformations of $\sigma_{k}$ is to show that we can find a nearby admissible conformal structure for which the 
hypothesis of Proposition 4.3 are satisfied. Thus by 4.3 such an admissible conformal structure will produce an exact solution to the main equation (2.1).

Before deforming the $\sigma_{k}$ we need to introduce a topology on the space of admissible conformal structures. For $G_{1}=R_{1} D_{1}$ and $G_{2}=R_{2} D_{2}$, two dilations uniquely decomposed into rotational and centered dilation factors, we define the distance between $G_{1}$ and $G_{2}$ to be

$$
d\left(G_{1}, G_{2}\right)=\max \left\{\left\|R_{1}-R_{2}\right\|,\left|q_{1}-q_{2}\right|,\left|\log \frac{\lambda_{1}}{\lambda_{2}}\right|\right\}
$$

where $q_{i}$ is the source and $\lambda_{i}$ is the strength of $G_{i}$, for $i=1,2$ respectively. The quantity $\left\|R_{1}-R_{2}\right\|$ denotes the operator norm of $R_{1}-R_{2}$ thought of as a linear transformation of $\mathbb{R}^{n+1}$. If $\sigma_{k}$ and $\bar{\sigma}_{k}$ are two admissible conformal structures on $\mathcal{T}_{k}$ then we define the distance between $\sigma_{k}$ and $\bar{\sigma}_{k}$ to be

$$
d\left(\sigma_{k}, \bar{\sigma}_{k}\right)=\max _{i}\left(\max \left\{d\left(G_{-i}, \bar{G}_{-i}\right), d\left(G_{+i}, \bar{G}_{+i}\right)\right\}\right)
$$

where the dilations $\left\{G_{ \pm i}\right\}$, (respectively $\left\{\bar{G}_{ \pm i}\right\}$ ) are those which correspond to $\sigma_{k}$, (respectively $\bar{\sigma}_{k}$ ). Note that $\sigma_{k}$ is defined either by specifying the dilations $G_{ \pm i}$ for $i=1, \ldots, k$ directly, or by specifying the conformal maps $F_{i}$ for $i=1, \ldots, k$ whose compositions generate most of the dilations and in addition specifying the dilations $G_{-1}$ and $G_{+k}$ independently. Thus one can easily see that $d\left(\sigma_{k}, \bar{\sigma}_{k}\right)=0$ if and only if $\sigma_{k}=\bar{\sigma}_{k}$, in the sense that $G_{ \pm i}=\bar{G}_{ \pm i}$ for all $i=1, \ldots, k$. It is then clear that $d(\cdot, \cdot)$ defines a distance function on the space of all conformal structures on $\mathcal{T}_{k}$.

Let $\mathcal{S}_{k}$ denote the space of admissible conformal structures on $\mathcal{T}_{k}$, with the topology that $\mathcal{S}_{k}$ inherits from the distance function $d(\cdot, \cdot)$ defined above. If we fix $k$ and suppress the index, then for any $\sigma_{1} \in \mathcal{S}$ and any $\delta>0$, we define the set $\mathcal{S}_{\delta}\left(\sigma_{1}\right) \subset \mathcal{S}$ by

$$
\mathcal{S}_{\delta}\left(\sigma_{1}\right)=\left\{\sigma \in \mathcal{S}: d\left(\sigma, \sigma_{1}\right)<\delta\right\}
$$

In $\S 3.5$ we constructed a specific metric $g_{1}\left(\sigma_{k}\right)$ corresponding to a given $\sigma_{k} \in$ $\mathcal{S}_{k}$, which has served as our approximate solution. Given an admissible conformal structure $\sigma_{k} \in \mathcal{T}_{k}$ we will call a metric $g$ on $M$ compatible with $\sigma_{k}$ if it is conformally equivalent to the metric $g_{1}\left(\sigma_{k}\right)$ constructed in $\S 3.5$. For each 
$\sigma \in \mathcal{S}_{k}$ let $\mathcal{M}_{\sigma}$ denote the space of compatible metrics. We then define $\mathcal{M}$ by

$$
\mathcal{M}=\bigcup_{\sigma \in \mathcal{T}_{k}} \mathcal{M}_{\sigma}
$$

Thus for any metric $g \in \mathcal{M}$ there exists an admissible conformal structure $\sigma=$ $\sigma(g)$ and a metric $g_{1}(\sigma)$ which is conformally equivalent to $g$, such that $g_{1}(\sigma)$ is an approximate solution. Given any $g \in \mathcal{M}$ we use the generalized Pohozaev identity to associate to $g$ a set of parameters which measure the extent to which $g$ fails to satisfy the hypothesis of Theorem 4.3. These parameters will then be used to specify deformations of given admissible conformal structures. Before defining our parameter space we need to review some facts about the conformal group of $S^{n}$. The following section is essentially from [15], for completeness and in order to establish our notation we include it here.

5.2. The Conformal Group of $S^{n}$. Recall that Minkowski $(\mathrm{n}+2)$-space, $\mathbf{M}^{n+2}$, is $\left(\mathbb{R}^{n+2},\langle\langle\cdot, \cdot\rangle\rangle\right)$, where $\langle\langle\cdot, \cdot\rangle\rangle$ is the Lorentz inner product

$$
\langle\langle y, z\rangle\rangle=\sum_{i=1}^{n+1} y_{i} z_{i}-y_{n+2} z_{n+2} .
$$

We view $S^{n}$ as the set of points $y$ in $\mathbf{M}^{n+2}$ satisfying $\sum_{i=1}^{n+1} y_{i}^{2}=y_{n+2}^{2}=1$. The Lie group $O(n+1,1) \subset G L(n+2, \mathbb{R})$ is the linear isometry group of bold $M^{n+2}$, i.e. it is the set of invertible $(n+2) \times(n+2)$ matrices which preserve the Lorentz inner product

$$
O(n+1,1)=\{A \in G L(n+2, \mathbb{R}):\langle\langle A y, A z\rangle\rangle=\langle\langle y, z\rangle\rangle\} .
$$

The group $O(n+1,1)$ acts on the sphere $S^{n}$ by

$$
A(y)=(A y)_{n+2}^{-1} A y .
$$

Under this action $O(n+1,1)$ represents the conformal group of $S^{n}$. The Lie algebra $o(n+1,1)$ of $O(n+1,1)$, consists of $(n+2) \times(n+2)$ matrices $X$ of the form

$$
X=\left(\begin{array}{cc}
X_{0} & \mathbf{w}^{t} \\
\mathbf{w} & 0
\end{array}\right),
$$

where $X_{0} \in o(n+1)$, the Lie algebra of the orthogonal group $O(n+1)$, $\mathbf{w}=\left(\mathbf{w}_{1}, \ldots, \mathbf{w}_{n+1}\right) \in \mathbb{R}^{n+1}$ is an vector, and $\mathbf{w}^{t}$ is its transpose. Thus $X_{0}$ is a skew symmetric matrix which we refer to as the rotational part of $X$ and 
the vector $\mathbf{w} \in \mathbb{R}^{n+1}$ is referred to as the pure dilational part of $X$. By an abuse of notation we write $X=X_{0}+\mathbf{w}$.

The Killing form $B: o(n+1,1) \times o(n+1,1) \rightarrow \mathbb{R}$ is the nondegenerate symmetric quadratic form given by

$$
\begin{aligned}
B(X, \hat{X}) & =-\sum_{1 \leq i<j \leq n+1} x_{i j} \hat{x}_{i j}+\sum_{i=1}^{n+1} x_{i n+2} \hat{x}_{i n+2} \\
& =\frac{1}{2} \operatorname{Tr}\left(X_{0} \hat{X}_{0}\right)+\mathbf{w} \cdot \hat{\mathbf{w}},
\end{aligned}
$$

where we have written $X$ and $\hat{X}$ in terms of their rotational and pure dilational components, and $\mathbf{w} \cdot \hat{\mathbf{w}}$ denotes the Euclidean inner product in $\mathbb{R}^{n+1}$. If we decompose $o(n+1,1)=o(n+1) \oplus \mathbb{R}^{n+1}$, as above, then we see that $B(\cdot, \cdot)$ is positive definite on $\mathbb{R}^{n+1}$ and negative definite on $o(n+1)$. Since $B$ is nondegenerate, it provides an identification of $o(n+1,1)$ with its dual space. If $X$ is an element of $o(n+1,1)$, we let $X^{*}$ denote the linear functional given by

$$
X^{*}(Y)=B(X, Y)
$$

It is through this identification that we shall use the boundary integrals (4.3) to attach to a each oriented edge of $\mathcal{T}_{k}$ an element of the dual of $o(n+1,1)$. These elements will in turn give rise to a collection of parameters corresponding to a particular compatible metric for some admissible conformal structure.

5.3. The Parameter Space and Evaluation Map. We define the parameter space $\mathcal{X}$ as follows. First fix a background balanced admissible conformal structure, $\sigma_{0}$ on $\mathcal{T}_{k}$. We may, for example let $\sigma_{0}$ be the conformal structure constructed in $\S 2.6$. Let $E$ denote the set of oriented edges of $\mathcal{T}_{k} . \quad E$ has cardinality $2 k$ and is given explicitly by

$$
E=\{ \pm 1, \ldots, \pm k\} .
$$

For each vertex $i=1, \ldots, k$ we define a hyperplane $W_{i} \subset \mathbb{R}^{n+1}$ by

$$
W_{i}=\left\{p \in \mathbb{R}^{n+1}: p \cdot q_{+i}=0\right\},
$$

where $q_{+i}$ is the source of the dilation $G_{+i}$ in the balanced admissible conformal structure $\sigma_{0}$. Note that the balancing condition implies that $q_{-i}+q_{+i}=0$, 
thus $W_{i}$ could be defined with respect to $q_{-i}$ as well. We define two spaces $\mathcal{X}_{1}$ and $\mathcal{X}_{2}$ by

$$
\mathcal{X}_{1}=\prod_{i=1}^{k} W_{i} \quad \mathcal{X}_{2}=\prod_{e \in E} \mathbb{R}=\mathbb{R}^{|E|}
$$

The parameter space $\mathcal{X}$ is then defined by

$$
\mathcal{X}=\mathcal{X}_{1} \times \mathcal{X}_{2}
$$

A point $(b, \mu) \in \mathcal{X}$ has components $b_{i} \in W_{i} \subset \mathbb{R}^{n+1}$ for $i=1, \ldots k$, and $\mu_{e} \in \mathbb{R}$ for $e \in E$. Given a pair $\delta=\left(\delta_{1}, \delta_{2}\right) \in \mathbb{R}^{+} \times \mathbb{R}^{+}$we define $\mathcal{X}_{\delta}(b, \mu)$ by

$\mathcal{X}_{\delta}(b, \mu)=\left\{(c, \nu) \in \mathcal{X}:\left|b_{i}-c_{i}\right| \leq \delta_{1}, \forall i=1, \ldots, k,\left|\nu_{e}-\mu_{e}\right| \leq \delta_{2}, \forall e \in E\right\}$

Given any admissible conformal structure $\sigma \in \mathcal{S}_{k}$, and any compatible metric $g \in \mathcal{M}_{\sigma}$ we define a linear functional $X^{*}(\sigma, g)_{e}$, on $o(n+1,1)$, for each $e \in E$ by

$$
X^{*}(\sigma, g)_{+i}(X)=-k(n)^{-1} \int_{F_{i}^{-1}\left(S_{+i}\right)}\left(\operatorname{Ric}_{g}-n^{-1} R g\right)\left(d F_{i}^{-1}(X), \nu\right) d \sigma_{g}
$$

for any $X \in o(n+1,1)$, where $k(n)=2(n-1)(n-2), \nu$ denotes the outward unit normal vector, with respect to $g$, from $\Omega_{i}$, and we've chosen $e=+i$. $X^{*}(\sigma, g)_{-i}(X)$ is defined similarly. This defines for each edge $e \in E$ an element of the dual of $o(n+1,1)$. We let $X(\sigma, g)_{e}$ denote the element of $o(n+1,1)$ which is associated to $X^{*}(\sigma, g)_{e}$ under the identification given by $B(\cdot, \cdot)$. Thus $X(\sigma, g)_{e}$ is defined by the property that for all $X \in o(n+1,1)$

$$
X^{*}(\sigma, g)_{e}(X)=B\left(X(\sigma, g)_{e}, X\right)
$$

Proposition 4.2 shows that $X\left(\sigma, g_{1}\right)_{+i}=\epsilon_{+i}^{n-2} q_{+i}$, where $g_{1}$ is the compatible metric constructed in $\S 2.5$. Thus $X\left(\sigma, g_{1}\right)_{+i}$ has no rotational component, and its pure dilational component is $\epsilon_{+i}^{n-2} q_{+i}$. For any compatible metric $g$ the elements $X(\sigma, g)_{+i}$ and $X(\sigma, g)_{-i}$ are determined by boundary integrals over the same hypersurface. The following Lemma shows that they are related to one another by the adjoint representation. We refer to [15, Lemma 2.2] for the proof. 
Lemma 5.1. For any $\sigma \in \mathcal{S}$, and any compatible metric $g$ we have

$$
X(\sigma, g)_{+i}=-\operatorname{Ad}\left(G_{-i}\right)\left(X(\sigma, g)_{-i}\right) .
$$

In particular, $B\left(X(\sigma, g)_{+i}, X(\sigma, g)_{+i}\right)=B\left(X(\sigma, g)_{-i}, X(\sigma, g)_{-i}\right)$.

For each vertex $i=1, \ldots, k$ we define $X(\sigma, g)_{i} \in o(n+1,1)$ by

$$
X(\sigma, g)_{i}=X(\sigma, g)_{-i}+X(\sigma, g)_{+i} .
$$

Note that if $\sigma_{0}$ is the balanced admissible conformal structure constructed in $\S 2.6$ and $g_{1}$ the compatible metric constructed in $\S 2.5$ with respect to $\sigma_{0}$, then $X\left(\sigma_{0}, g_{1}\right)_{i}=0$ for each $i=1, \ldots, k$. We express $X(\sigma, g)_{i}$ in terms of its rotational and pure dilational components by writing

$$
X(\sigma, g)_{i}=X^{0}(\sigma, g)_{i}+C(\sigma, g)_{i},
$$

where $X^{0}(\sigma, g)_{i} \in o(n+1)$ and $C(\sigma, g)_{i} \in \mathbb{R}^{n+1}$.

We define the evaluation map

$$
\Pi: \mathcal{M} \rightarrow \mathcal{X}
$$

by setting $\Pi(\sigma, g)=(b, \mu)$, where $b$ and $\mu$ are described as follows. The components $b_{i} \in W_{i}$ of $b \in \mathcal{X}_{1}$ are defined by

$$
b_{i}=\epsilon^{n-2} \mathbf{P}_{i}\left(C(\sigma, g)_{i}\right),
$$

where $\mathbf{P}_{i}: \mathbb{R}^{n+1} \rightarrow W_{i}$ is the orthogonal projection operator. To define the components $\mu_{e}$ of $\boldsymbol{\mu} \in \mathcal{X}_{2}$, we first attach a weight $\lambda_{e}(\sigma, g)$ to each edge $e \in E$ by setting

$$
\lambda_{e}(\sigma, g)=\left[B\left(X(\sigma, g)_{e}, X(\sigma, g)_{e}\right)\right]^{\frac{1}{2-n}},
$$

provided the term in brackets is positive, and zero otherwise. In all the situations we encounter, $B\left(X(\sigma, g)_{e}, X(\sigma, g)_{e}\right)$ will be positive, this is because will consider only conformal maps with strong dilational components and weak rotational components. Observe that Lemma 5.1 implies that $\lambda_{-i}(\sigma, g)=$ $\lambda_{+i}(\sigma, g)$. Proposition 4.2 implies that for $e=+i \lambda_{e}\left(\sigma_{0}, g_{1}\right)=\lambda_{+i}$, the strength of $G_{+i}$. The element $\boldsymbol{\mu} \in \mathcal{X}_{2}$ is then defined by setting

$$
\mu_{e}=\log \left(\frac{\lambda_{e}(\sigma, g)}{\lambda_{e}\left(\sigma_{0}, g_{1}\right)}\right)
$$


for each $e \in E$, where $\left(\sigma_{0}, g_{1}\right)$ is the fixed balanced admissible conformal structure and compatible metric constructed in $\S 2.5-2.6$. Notice that $\Pi\left(\sigma_{0}, g_{1}\right)=$ $(0,0)$.

5.4. Deformations of Conformal Structures. The deformation map will assign to any element in the parameter space, $(b, \mu)$, which is close to $(0,0)$, the parameters which come from $\left(\sigma_{0}, g_{1}\right) \in \mathcal{M}$, a new admissible conformal structure $\mathcal{D}(b, \mu)$ which is close to $\sigma_{0}$. Thus if $\delta_{1}, \delta_{2}$ and $\delta_{3}$ are small and $\delta=\left(\delta_{1}, \delta_{2}\right)$, then $\mathcal{D}$ will satisfy

$$
\mathcal{D}: \mathcal{X}_{\delta}(0,0) \rightarrow \mathcal{S}_{\delta_{3}}\left(\sigma_{0}\right)
$$

The map $\mathcal{D}$ will have the additional property that if $\mathcal{D}(b, \mu)=\sigma$ and $g_{1}$ denotes the metric canonically constructed from $\sigma$ as in $\S 2.6$ then $\Pi\left(\mathcal{D}(b, \mu), g_{1}\right)=$ $\Pi\left(\sigma, g_{1}\right)$ is very close to $(b, \mu)$. This says that when we evaluate the parameters arising from the deformed conformal structure, with the canonical metric associated to it, we get parameters which are close to the parameters we used to specify the deformation. Before defining the deformation map itself we first discuss how to deform the centers of mass and the edge weights independently.

5.4.1. Deformations of Centers of Mass. Given a set of points $q_{1}, \ldots, q_{l}$ in $S^{n}$ and weights $a_{1}, \ldots, a_{l}$ in $\mathbb{R}^{+}$, the weighted center of mass is the sum

$$
C=\sum_{j=i}^{l} a_{j} q_{j}
$$

The balancing condition is that the weighted center of mass vanishes, here $q_{-i}$ and $q_{+i}$ are the sources of the dilations $G_{-i}$ and $G_{+i}$, and the weights are $\epsilon_{-i}^{n-2}$ and $\epsilon_{+i}^{n-2}$. So the balancing condition becomes

$$
\epsilon_{-i}^{n-2} q_{-i}+\epsilon_{+i}^{n-2} q_{+i}=0,
$$

so that $\epsilon_{-i}=\epsilon_{+i}$ and $q_{-i}=-q_{+i}$. Therefore to specify a balanced admissible conformal structure we need only specify at each vertex $i$ a point $q_{i} \in S^{n}$ and a weight $a_{i} \in R^{+}$satisfying $\beta_{1}^{-1} \leq a_{i} \leq \beta_{1}$ for a constant $\beta_{1}$.

Fix $q_{1} \in S^{n}$, and $a_{1}$ such that $\beta^{-1} \leq a_{1} \leq \beta$, and let $W=\left\{p \in \mathbb{R}^{n+1}\right.$ : $\left.p \cdot q_{1}=0\right\}$. For any $p \in B^{n+1}$ let $F_{p}: B^{n+1} \rightarrow B^{n+1}$ be the unique centered 
dilation with $F_{p}(0)=p$. We define a map $C: B^{n+1} \cap W \rightarrow W$ by

$$
C(p)=a_{1} F_{p}\left(q_{1}\right)+a_{1} F_{p}\left(-q_{1}\right) .
$$

The following lemma will allow us to use this map to produce deformations of the centers of mass which are approximately prescribed by the parameters $b_{i} \in \mathcal{X}_{1}$. We refer to [15, Lemma 2.3] for the proof.

Lemma 5.2. The map $C: B^{n+1} \cap W \rightarrow W$ is smooth and defines a diffeomorphism from a neighborhood $B_{\sigma_{1}}^{n+1} \cap W=\left\{p \in W:|p|<\sigma_{1}\right\}$ of 0 onto a neighborhood of 0 containing $B_{\sigma_{2}}^{n+1} \cap W$, where $\sigma_{1}, \sigma_{2}$ depend only on $n, \beta$.

5.4.2. Deformations of Edge Weights. Given any assignment of weights to the edges of $\mathcal{T}$, we achieve this exact assignment by a deformation of $\sigma_{0}$. Let $\bar{\lambda}=\left\{\bar{\lambda}_{e}\right\} \in \mathcal{X}_{2}$ be an assignment of edge weights with $\beta_{2} \bar{\lambda}_{e} \leq \lambda_{e} \leq \beta_{2} \bar{\lambda}_{e}$ for a constant $\beta_{2}$ and for all edges $e \in E$. Here $\lambda_{e}=\lambda_{e}\left(\sigma_{0}, g_{1}\right)$ is weight assigned to the edge $e$ by the balanced conformal structure $\sigma_{0}$. We define $\sigma(\bar{\lambda})$ by defining the dilations $G_{e}(\bar{\lambda})$ by defining for $G_{-i}(\bar{\lambda})$ by

$$
G_{-i}(\bar{\lambda})=R_{-i} D_{-i}(\bar{\lambda}),
$$

where $G_{-i}=R_{-i} D_{-i}$ is the corresponding dilation for $\sigma_{0}$, and $D_{-i}(\bar{\lambda})$ is the centered dilation with the same fixed points as $D_{-i}$ but with strength $\bar{\lambda}_{-i}$. The dilations $G_{+i}(\bar{\lambda})$ are defined in the same way. Thus if $\bar{\lambda}=\lambda=\left\{\lambda_{ \pm i}\right\}$ are the weights of $\sigma_{0}$, we have $D_{ \pm i}\left(\lambda_{ \pm i}\right)=D_{ \pm i}$, and $\sigma(\lambda)=\sigma_{0}$.

5.4.3. The Deformation Map. We are now in a position to define the deformation map $\mathcal{D}$. We shall initially deform the background balanced admissible conformal structure $\sigma_{0}$ on $\mathcal{T}_{k}$, as dictated by a given set of parameters. Given $(b, \mu) \in \mathcal{X}_{\delta}(0,0)$, we define $\sigma=\mathcal{D}(b, \mu)$ as follows. From each of the parameters $b_{i} \in b$ we specify a centered dilation in the following way. Let $a_{i}=\epsilon^{n-2} \epsilon_{-i}^{n-2}$ (thus by the balancing condition $a_{i}=\epsilon^{n-2} \epsilon_{+i}^{n-2}$ as well). By applying Lemma 5.2 to the vectors $q_{-i}$ and $q_{+i}$ with the weight $a_{i}$ we find that there is a unique point $p_{i} \in B_{\sigma_{1}}^{n+1} \cap W_{i}$ such that $C\left(p_{i}\right)=b_{i}$. Thus we get a unique (small) centered dilation $D_{i}=F_{p_{i}}$, such that

$$
\begin{aligned}
b_{i} & =a_{i}\left[D_{i}\left(q_{-i}\right)+D_{i}\left(q_{+i}\right)\right] \\
& =\epsilon^{n-2} \epsilon_{-i}\left[D_{i}\left(q_{-i}\right)+D_{i}\left(-q_{-i}\right)\right] .
\end{aligned}
$$


The parameters $\left\{\mu_{e}\right\}=\mu$ are used to produce a change in the dilation strength along each edge, which when coupled with the change in the center of mass at each vertex $i$ prescribed by $\left\{b_{i}\right\}=b$ as above will define the deformation $\mathcal{D}$. The admissible conformal structure $\sigma$ will be specified by defining the dilations $G_{ \pm i}^{(\sigma)}$ by

$$
G_{-i}^{(\sigma)}=D_{i-1} \cdot G_{-i}^{(\sigma(\bar{\lambda}))} \cdot D_{i}^{-1} \quad \text { and } \quad G_{+i}^{(\sigma)}=D_{i+1} \cdot G_{+i}^{(\sigma(\bar{\lambda}))} \cdot D_{i}^{-1}
$$

where $G_{ \pm i}^{(\sigma(\bar{\lambda}))}=G_{ \pm i}(\bar{\lambda})$ as above, and $\bar{\lambda}$ is an assignment of edge weights chosen so that

$$
\mu_{-i}=\log \frac{\lambda_{-i}(\sigma)}{\lambda_{-i}\left(\sigma_{0}\right)} \quad \text { and } \quad \mu_{+i}=\log \frac{\lambda_{+i}(\sigma)}{\lambda_{+i}\left(\sigma_{0}\right)}
$$

Here $\lambda_{ \pm i}(\sigma)$ are the strengths of the dilations $G_{ \pm i}^{(\sigma)}$ from our new conformal structure $\sigma$, and $\lambda_{ \pm i}\left(\sigma_{0}\right)$ are the strengths of the dilations $G_{ \pm i}^{\left(\sigma_{0}\right)}$ from our background balanced conformal structure $\sigma_{0}$. In [15, 345-346] it is shown that there is a unique choice of $\bar{\lambda}_{-i}$ and $\bar{\lambda}_{+i}$ which are close in ratio to $\lambda_{i}\left(\sigma_{0}\right)$ and $\lambda_{+i}\left(\sigma_{0}\right)$ respectively, such that (5.4) holds. Note that the deformation $\sigma_{0} \rightarrow \sigma(\bar{\lambda})$ only produces a change in the dilation strength along each edge. Our choice for the dilations $G_{ \pm i}^{(\sigma)}$ insures that the conformal transformations $F_{i}^{(\sigma)}$ attached by $\sigma$ to each vertex $i$ of $\mathcal{T}_{k}$ satisfy

$$
F_{i}^{(\sigma)}=D_{i} \cdot F_{i}^{(\sigma(\bar{\lambda}))}
$$

This completes the definition of the deformation map $\mathcal{D}$. It is immediate from the construction that $\mathcal{D}$ is continuous with respect to the product topology on $\mathcal{X}$ and the topology induced on $\mathcal{S}$ by the distance function $d$ defined above. The degree to which $\Pi\left(\mathcal{D}(b, \mu), g_{1}\right)$ is close to $(b, \mu)$ for any $(b, \mu) \in \mathcal{X}_{\delta}(0,0)$ is expressed by the following Proposition.

Proposition 5.3. Let $\delta=\left(\delta_{1}, \delta_{2}\right)$, where $\delta_{1}, \delta_{2}$ are small positive numbers. We then have $\mathcal{D}\left(\mathcal{X}_{\delta}(0,0)\right) \subset \mathcal{S}_{\delta_{3}}\left(\sigma_{0}\right)$, where $\delta_{3}=c \max \left\{\delta_{1}^{1 / 2}, \delta_{2}\right\}$, c depending only on $n$ and $\beta$. If $(b, \mu) \in \mathcal{X}_{\delta}(0,0)$, and we denote $\mathcal{D}(b, \mu)=\sigma$, then we have $\Pi\left(\sigma, g_{1}\right)=(b(\sigma), \mu(\sigma))$, where $\mu(\sigma)=\mu$, and $b(\sigma)$ satisfies

$$
\left|b_{i}(\sigma)-b_{i}\right| \leq c\left(\epsilon^{2} \delta_{1}^{1 / 2}+\delta_{2}\right)
$$

for a constant $c$ depending only on $n$ and $\beta$. 
This is identical (verbatim) to [15, Proposition 2.4], and hence we refer there for the proof.

5.5. Deforming Within a Fixed Conformal Class. For each approximate solution $g_{1_{k}}$, we have constructed a solution of the projected problem (3.1). The $N$ distinct approximate solution metrics $g_{1_{1}}, \ldots, g_{1_{N}}$, derived from the background balanced conformal $N$ structure, $\sigma_{0}(N)$, all lie within a fixed conformal class. Thus the $N$ distinct solutions to each of the projected problems arising from the metrics $g_{1_{1}}, \ldots, g_{1_{N}}$ also lie within this fixed conformal class. In the next section we show that for each of these projected problems we may find an appropriate deformation of the background balanced admissible conformal structure $\sigma_{0_{k}}$ so that the hypothesis of Proposition 4.3 are satisfied, and hence the solution of the projected problem with respect to this new admissible conformal structure is actually an exact solution to the main equation (2.1). In order for this procedure to produce $N$ solutions to (2.1) in a fixed conformal class, as we require, it will be necessary to show that the required deformations actually preserve the conformal class. This is done by associating to each deformation $\sigma_{0_{k}} \rightarrow \sigma_{k}$ a conformal transformation of the nondegenerate end sphere $\left(S^{n}, \bar{g}_{0}\right)$ and then by adjusting the decomposition (1.7) accordingly, we show that the two approximate solution metrics $g_{1_{k}}=g_{1}\left(\sigma_{0_{k}}\right)$ and $g_{1}\left(\sigma_{k}\right)$ are conformally equivalent.

To emphasize the dependence of the decomposition (1.7) on the conformal structure we fix $k, 1 \leq k \leq N$ and write

$$
M=M_{0} \cup_{F_{0}^{\left(\sigma_{0}\right)}} \Omega_{\sigma_{0}} \cup_{F_{k+1}^{\left(\sigma_{0}\right)}} S_{0}^{n}
$$

for the decomposition of $M$ with respect to the conformal structure $\sigma_{0}$ on $\mathcal{T}_{k}$, and

$$
M=M_{0} \cup_{F_{0}^{(\sigma)}} \Omega_{\sigma} \cup_{F_{k+1}^{(\sigma)}} S_{0}^{n}
$$

for the decomposition of $M$ with respect to the conformal structure $\sigma$ on $\mathcal{T}_{k}$. The subdomains $M_{0}$ and $S_{0}^{n}$ appearing in the decompositions (5.5) and (5.6) also depend on the conformal structure. We obtain $M_{0}$ by removing from $M$ the inverse image of a small ball $B_{0}$ in $S^{n}$ under the conformal map $\Phi_{M}$ from 
a domain in $(M, g)$ to a domain in $\left(S^{n}, g_{0}\right)$,

$$
M_{0}=M \backslash \Phi_{M}^{-1}\left(B_{0}\right) .
$$

The ball $B_{0}$ is determined directly by the dilation $G_{-1}$, namely

$$
\partial B_{0}=\left\{p:\left|G_{-1}^{-1^{\prime}}(p)\right|=1\right\} .
$$

Thus the subdomain $M_{0}$ in (5.5) is determined by $G_{-1}^{\left(\sigma_{0}\right)}$, while the corresponding subdomain in (5.6) is determined by $G_{-1}^{(\sigma)}$. In view of the definition of our deformation map $\mathcal{D}$, we need only consider conformal structures $\sigma$ which are close to $\sigma_{0}$, in particular we assume that $d\left(\sigma, \sigma_{0}\right)<\delta_{3}=c \max \left\{\delta_{1}^{1 / 2}, \delta_{2}\right\}$. This implies that the balls $B_{0}\left(\sigma_{0}\right)$ and $B_{0}(\sigma)$ are close to each other, since their centers $-q_{-1}\left(\sigma_{0}\right)$ and $-q_{-1}(\sigma)$ satisfy

$$
\left|\left(-q_{-1}\left(\sigma_{0}\right)\right)-\left(-q_{-1}(\sigma)\right)\right| \leq \delta_{3},
$$

and the strength of the dilations $G_{-1}^{\left(\sigma_{0}\right)}$ and $G_{-1}^{(\sigma)}$ which determine them satisfy

$$
\left|\log \left(\lambda_{-1}^{\left(\sigma_{0}\right)} / \lambda_{-1}^{(\sigma)}\right)\right| \leq \delta_{3}
$$

The subdomain $S_{0}^{n}$ is obtained in the same manner as above, with the corresponding small balls $B_{k+1}\left(\sigma_{0}\right)$ and $B_{k+1}(\sigma)$ being determined by the dilations $G_{+k}^{\left(\sigma_{0}\right)}$ and $G_{+k}^{(\sigma)}$ respectively. We now use the closeness of these balls to rewrite the decompositions (5.5) and (5.6) in such a way that the ends are independent of the conformal structure, and thus isometric to one another when endowed with the approximate solution metrics $g_{1}\left(\sigma_{0}\right)$ and $g_{1}(\sigma)$.

Recall that for some $r>0,(M, g)$ and $\left(S^{n}, \bar{g}_{0}\right)$ are locally conformally flat in a ball of radius $r$ about some point $x_{0} \in M$, and $y_{0} \in S^{n}$. It is about these distinguished points that we've attached the string of $k$ asr's, $\Omega$. We shall assume that $\delta_{3}$ is arbitrarily small relative to $r$, this will hold provided the same is true of $\delta_{1}$ and $\delta_{2}$, i.e. if we restrict our deformations to a neighborhood of $(0,0) \in \mathcal{X}$, which is small relative to $r$. Thus there exists a small constant $c_{1}$ such that

$$
\Phi_{M}^{-1}\left(B_{0}\right) \subset B_{c_{1}}\left(x_{0}\right) \subset B_{r}\left(x_{0}\right) \subset M \text { and } \Phi_{S^{n}}^{-1}\left(B_{k+1}\right) \subset B_{c_{1}}\left(y_{0}\right) \subset B_{r}\left(y_{0}\right) \subset S^{n},
$$

where in $M$ these are geodesic balls with respect to $g$, and in $S^{n}$ with respect to $\bar{g}_{0}$, and $B_{0}$ and $B_{k+1}$ are the balls described above for any admissible conformal 
structure $\sigma \in \mathcal{S}_{\delta_{3}}\left(\sigma_{0}\right)$. We define the subdomains $M_{0}^{-}$and $\left(S_{0}^{n}\right)^{-}$by

$$
M_{0}^{-}=M \backslash B_{c_{1}}\left(x_{0}\right) \quad \text { and } \quad\left(S_{0}^{n}\right)^{-}=S^{n} \backslash B_{c_{1}}\left(y_{0}\right) .
$$

If we use these subdomains in place of $M_{0}$ and $S_{0}^{n}$, the decompositions (5.5) and (5.6) become

$$
M=M_{0}^{-} \cup \Omega_{\sigma_{0}}^{+} \cup\left(S_{0}^{n}\right)^{-},
$$

for the decomposition with respect to $\sigma_{0}$ and

$$
M=M_{0}^{-} \cup \Omega_{\sigma}^{+} \cup\left(S_{0}^{n}\right)^{-},
$$

for the decomposition with respect to $\sigma$. The domains $\Omega_{\sigma_{0}}^{+}$and $\Omega_{\sigma}^{+}$which contain the $k$ asr's are given by

$$
\begin{aligned}
\Omega_{\sigma_{0}}^{+} & =\left\{M_{0}\left(\sigma_{0}\right) \backslash M_{0}^{-}\right\} \cup_{F_{0}^{\left(\sigma_{0}\right)}} \Omega_{\sigma_{0}} \cup_{F_{k+1}^{\left(\sigma_{0}\right)}}\left\{S_{0}^{n}\left(\sigma_{0}\right) \backslash\left(S_{0}^{n}\right)^{-}\right\} \\
\Omega_{\sigma}^{+} & =\left\{M_{0}(\sigma) \backslash M_{0}^{-}\right\} \cup_{F_{0}^{(\sigma)}} \Omega_{\sigma} \cup_{F_{k+1}^{(\sigma)}}\left\{S_{0}^{n}(\sigma) \backslash\left(S_{0}^{n}\right)^{-}\right\}
\end{aligned}
$$

In the decompositions (5.7) and (5.8) the subdomains $M_{0}^{-}$and $\left(S_{0}^{n}\right)^{-}$do not depend on the admissible conformal structure. Moreover, since $c_{1}$ is arbitrarily large relative to $\delta_{3}$ we see that when endowed with the approximate solution metrics, $\left(M_{0}^{-}, g_{1}\left(\sigma_{0}\right)\right)$ and $\left(M_{0}^{-}, g_{1}(\sigma)\right)$ are isometric as are $\left(\left(S_{0}^{n}\right)^{-}, g_{1}\left(\sigma_{0}\right)\right)$ and $\left(\left(S_{0}^{n}\right)^{-}, g_{1}(\sigma)\right)$. We have omitted the conformal maps $F_{0}$ and $F_{k+1}$ from the decompositions (5.7) and (5.8) because their role is in defining $\Omega_{\sigma_{0}}^{+}$and $\Omega_{\sigma}^{+}$, while the ends $M_{0}^{-}$and $\left(S_{0}^{n}\right)^{-}$are attached by the identity map.

The issue then becomes whether $\Omega_{\sigma_{0}}^{+}$and $\Omega_{\sigma}^{+}$are conformally equivalent. In general, they are not conformally equivalent, however we now show that by making a conformal deformation of $\left(S^{n}, \bar{g}_{0}\right)$ we can exhibit a final decomposition of $M$ with respect to $\sigma$ as

$$
M=M_{0}^{-} \cup \tilde{\Omega}_{\sigma}^{+} \cup\left(\tilde{S}_{0}^{n}\right)^{-},
$$

such that

$$
\begin{aligned}
\left(M_{0}^{-}, g_{1}\left(\sigma_{0}\right)\right) & =\left(M_{0}^{-}, g_{1}(\sigma)\right) \\
\left(\Omega_{\sigma_{0}}^{+}, g_{1}\left(\sigma_{0}\right)\right) & \cong\left(\tilde{\Omega}_{\sigma}^{+}, g_{1}(\sigma)\right) \\
\left(\left(S_{0}^{n}\right)^{-}, g_{1}\left(\sigma_{0}\right)\right) & \cong\left(\left(\tilde{S}_{0}^{n}\right)^{-}, g_{1}(\sigma)\right)
\end{aligned}
$$


where $=$ denotes an isometry and $\cong$ denotes a conformal equivalence. Note that the isometry is immediate since we do not change the role of $M_{0}^{-}$in the decomposition. The conformal equivalences are possible because $\left(\Omega_{\sigma_{0}}^{+}, g_{1}\left(\sigma_{0}\right)\right)$ and $\left(\Omega_{\sigma}^{+}, g_{1}(\sigma)\right)$ are each conformally equivalent to Euclidean annuli, $A_{R}=$ $\{x: 1 \leq|x| \leq R\}$, and any two annuli are conformally equivalent if and only if they have the same conformal modulus. Let $R_{0}$ and $R(\sigma)$ be the two radii such that

$$
\begin{aligned}
\left(\Omega_{\sigma_{0}}^{+}, g_{1}\left(\sigma_{0}\right)\right) & \cong A_{R_{0}} \\
\left(\Omega_{\sigma}^{+}, g_{1}(\sigma)\right) & \cong A_{R(\sigma)} .
\end{aligned}
$$

Since $d\left(\sigma, \sigma_{0}\right)<\delta_{3}$, we have $\left|R(\sigma)-R_{0}\right|<c \delta_{3}$, where $c=c(k)$. Any deformation $\sigma_{0} \rightarrow \sigma$, where $\sigma \in \mathcal{S}_{\delta_{3}}\left(\sigma_{0}\right)$ consists of deforming each dilation, say $G_{-i}^{\left(\sigma_{0}\right)}$ to one whose source is close to $q_{-i}\left(\sigma_{0}\right)$ and whose strength is close to $\lambda_{-i}\left(\sigma_{0}\right)$. Thus the net affect of these deformations is at most a small increase or decrease (according to whether the strengths of the dilations increase or decrease on average) in the conformal modulus, $\mathrm{cm}\left(\Omega_{\sigma_{0}}^{+}\right)$.

On $\left(S^{n}, \bar{g}_{0}\right)$ there exists a one parameter family of metrics $\bar{g}_{\tau} \in\left[\bar{g}_{0}\right]$ which are each isometric to $\bar{g}_{0}$ outside of a neighborhood of $y_{0} \in S^{n}$. These are constructed by using weak centered dilations on $\left(S^{n}, g_{0}\right)$. Consider the conformal map

$$
\Phi_{S^{n}}:\left(B_{r}\left(y_{0}\right), \bar{g}_{0}\right) \rightarrow\left(S^{n}, g_{0}\right) .
$$

Let $q=\Phi_{S^{n}}\left(y_{0}\right)$, and let $D_{\tau}$ denote the centered dilation with source $q$ and strength $\left|D_{\tau}^{\prime}(q)\right|=\tau$. We will use the maps $D_{\tau}$ when $\operatorname{cm}\left(\Omega_{\sigma_{0}}^{+}\right)>\operatorname{cm}\left(\Omega_{\sigma}^{+}\right)$ and when $\mathrm{cm}\left(\Omega_{\sigma_{0}}^{+}\right)<\mathrm{cm}\left(\Omega_{\sigma}^{+}\right)$we use the maps $D_{-\tau}=D_{\tau}^{-1}$. Assume that our deformation is such that $c m\left(\Omega_{\sigma_{0}}^{+}\right)>c m\left(\Omega_{\sigma}^{+}\right)$, i.e. $R_{0}>R(\sigma)$, the other case will be dealt with in a similar manner. The dilations $D_{\tau}$ give rise to a conformal map $\Psi_{\tau}$ of $\left(B_{2 c_{1}}\left(y_{0}\right), \bar{g}_{0}\right)$ into $\left(B_{r}\left(y_{0}\right), \bar{g}_{0}\right)$ defined by

$$
\Psi_{\tau}=\Phi_{S^{n}}^{-1} \cdot D_{\tau} \cdot \Phi_{S^{n}} \quad: \quad B_{2 c_{1}}\left(y_{0}\right) \rightarrow B_{r}\left(y_{0}\right) .
$$

Define the metric $g_{\tau}$ by

$$
g_{\tau}=\Psi_{\tau}^{*}\left(\bar{g}_{0}\right) \quad \text { in } \quad B_{2 c_{1}}\left(y_{0}\right)
$$


We require that $\tau$ be sufficiently small so that $\Psi_{\tau}\left(B_{2 c_{1}}\left(y_{0}\right)\right) \subset B_{r}\left(y_{0}\right)$. Since $\Psi_{\tau}$ is conformal $g_{\tau}=\psi_{\tau} \bar{g}_{0}$, for some $\psi_{\tau} \in C^{\infty}\left(B_{2 c_{1}}\left(y_{0}\right)\right), \psi_{\tau}>0$. Let $\xi$ be a smooth nonincreasing radial function about $y_{0}$ on $S^{n}$, i.e. $\xi(y)=\xi\left(d_{\bar{g}_{0}}\left(y, y_{0}\right)\right)$ which satisfies

$$
\xi(y)=\left\{\begin{array}{lll}
1 & \text { for } & y \in B_{c_{1}}\left(y_{0}\right) \\
0 & \text { for } & y \in B_{2 c_{1}}\left(y_{0}\right)
\end{array}\right.
$$

Here $d_{\bar{g}_{0}}\left(\cdot, y_{0}\right)$ denotes the distance function from $y_{0}$ taken with respect to $\bar{g}_{0}$, we assume that $2 c_{1}<i\left(S^{n}, \bar{g}_{0}\right)$, the injectivity radius, so that $\xi$ is well defined in $B_{2 c_{1}}\left(y_{0}\right)$ and hence on all of $S^{n}$. Define $\bar{\psi}_{\tau}$ by

$$
\bar{\psi}_{\tau}=\xi \psi_{\tau}+(1-\xi),
$$

then $\bar{\psi}_{\tau} \in C^{\infty}\left(S^{n}\right)$ and $\bar{\psi}_{\tau}>0$. Therefore the metric

$$
\bar{g}_{\tau}=\bar{\psi}_{\tau} \bar{g}_{0} \quad \text { on } \quad S^{n}
$$

is conformally equivalent to $\bar{g}_{0}$ on $S^{n}$ and satisfies

$$
\bar{g}_{\tau}=\left\{\begin{array}{lll}
g_{\tau} & \text { on } & B_{c_{1}}\left(y_{0}\right) \\
\bar{g}_{0} & \text { on } & S^{n} \backslash B_{2 c_{1}}\left(y_{0}\right)
\end{array}\right.
$$

Therefore we have

$$
\left(B_{c_{1}}\left(y_{0}\right), g_{\tau}\right)=\left(\Psi_{\tau}\left(B_{c_{1}}\left(y_{0}\right)\right), \bar{g}_{0}\right) .
$$

Thus since $\bar{g}_{\tau}$ and $\bar{g}_{0}$ are pointwise conformal on $S^{n}$, we let

$$
\left(\tilde{S}_{0}^{n}\right)^{-}=S^{n} \backslash \Psi_{\tau}\left(B_{c_{1}}\left(y_{0}\right)\right)
$$

and obtain

$$
\left(\left(S_{0}^{n}\right)^{-}, \bar{g}_{\tau}\right)=\left(\left(\tilde{S}_{0}^{n}\right)^{-}, \bar{g}_{0}\right)
$$

Thus

$$
\begin{aligned}
\left(\left(\tilde{S}_{0}^{n}\right)^{-}, g_{1}(\sigma)\right) & \cong\left(\left(S_{0}^{n}\right)^{-}, \bar{g}_{0}\right) \\
& =\left(\left(S_{0}^{n}\right)^{-}, g_{1}\left(\sigma_{0}\right)\right)
\end{aligned}
$$

as required. Let $A(\tau)=\Psi_{\tau}\left(B_{c_{1}}\left(y_{0}\right)\right) \backslash B_{c_{1}}\left(y_{0}\right)$ and define $\tilde{\Omega}_{\sigma}^{+}$by

$$
\begin{aligned}
\tilde{\Omega}_{\sigma}^{+} & =\left\{M_{0}(\sigma) \backslash M_{0}^{-}\right\} \cup_{F_{0}^{(\sigma)}} \Omega_{\sigma} \cup_{F_{k+1}^{(\sigma)}}\left\{S_{0}^{n}(\sigma) \backslash\left(S^{n} \backslash \Psi_{\tau}\left(B_{c_{1}}\left(y_{0}\right)\right)\right)\right\} \\
& =\Omega_{\sigma}^{+} \cup A(\tau) .
\end{aligned}
$$


For an appropriate choice of $\tau$ this will give the decomposition (5.9). When endowed with the approximate solution metric $g_{1}(\sigma), \tilde{\Omega}_{\sigma}^{+}$is the union

$$
\left(\tilde{\Omega}_{\sigma}^{+}, g_{1}(\sigma)\right)=\left(\Omega_{\sigma}^{+}, g_{1}(\sigma)\right) \cup\left(A(\tau), \bar{g}_{0}\right) .
$$

Since $\left(A(\tau), \bar{g}_{0}\right)$ is conformally equivalent to a Euclidean annulus we have

$$
\operatorname{cm}\left(\tilde{\Omega}_{\sigma}^{+}, g_{1}(\sigma)\right)=R(\sigma)+R(\tau),
$$

where $R(\tau)=c m\left(A(\tau), \bar{g}_{0}\right)$. Finally since $R_{0}-R(\sigma)<c \delta_{3}$ and $c_{1}$ while large relative to $\delta_{3}$ may be chosen arbitrarily small relative to $r$, we see that $\tau$ may be chosen in a sufficiently large continuum about zero to guarantee the existence of a $\tau$ such that

$$
R(\tau)=R_{0}-R(\sigma) .
$$

With this choice of $\tau$ we thus have

$$
\operatorname{cm}\left(\tilde{\Omega}_{\sigma}^{+}, g_{1}(\sigma)\right)=R_{0}
$$

and therefore

$$
\left(\tilde{\Omega}_{\sigma}^{+}, g_{1}(\sigma)\right) \cong\left(\Omega_{\sigma_{0}}^{+}, g_{1}\left(\sigma_{0}\right)\right)
$$

as required.

The following Theorem summarizes what we have shown.

Theorem 5.4. Given any admissible conformal structure $\sigma \in \mathcal{S}_{\delta_{3}}\left(\sigma_{0}\right)$, there exist decompositions of $M$,

$$
\begin{aligned}
& M=M_{0}^{-} \cup \Omega_{\sigma_{0}}^{+} \cup\left(S_{0}^{n}\right)^{-} \\
& M=M_{0}^{-} \cup \tilde{\Omega}_{\sigma}^{+} \cup\left(\tilde{S}_{0}^{n}\right)^{-}
\end{aligned}
$$

such that

$$
\begin{aligned}
\left(M_{0}^{-}, g_{1}\left(\sigma_{0}\right)\right) & =\left(M_{0}^{-}, g_{1}(\sigma)\right) \\
\left(\Omega_{\sigma_{0}}^{+}, g_{1}\left(\sigma_{0}\right)\right) & =\left(\tilde{\Omega}_{\sigma}^{+}, g_{1}(\sigma)\right) \\
\left(\left(S_{0}^{n}\right)^{-}, g_{1}\left(\sigma_{0}\right)\right) & =\left(\left(\tilde{S}_{0}^{n}\right)^{-}, g_{1}(\sigma)\right)
\end{aligned}
$$

provided that $\delta_{3}<c$ where $c=c(N, r)$ is a small constant. In particular $g_{1}(\sigma) \in\left[g_{1}\left(\sigma_{0}\right)\right]$, and thus the required deformations preserve the conformal class. 


\section{Deformation to Exact Solutions}

6.1. Controlled Deformations. In this section we solve the main equation (2.1) by finding a unique small deformation of the background balanced admissible conformal structure $\sigma_{0}$ on $\mathcal{T}_{k}$ to a new admissible conformal structure $\sigma \in \mathcal{S}_{\delta_{3}}\left(\sigma_{0}\right)$, such that the solution to the projected problem (3.1) corresponding to $\sigma$ is actually an exact solution of (2.1). This is done by choosing the deformation so that the solution $g_{k}$, of (3.1) corresponding to $\sigma$ satisfies the hypothesis of Proposition 4.3 and is therefore an exact solution of (2.1). Since it was shown in $\S 5.5$ that the deformations preserve the conformal class, by finding such a deformation for each $k=1, \ldots, N$ we produce $N$ distinct solutions in a fixed conformal class. It remains to show that these solutions lie in a conformal class which is an arbitrarily small perturbation in $C^{0}$ of our initial background conformal class $g$ on $M$. This is shown in the final section of this section, and thus completes the proof of our Main Theorem (0.1).

Before showing that such deformations to exact solutions exist we need to recall some results from [15] which indicate the extent to which the deformations we employ are "controlled". We fix a $k, 1 \leq k \leq N$, and let $g_{k}=(1+\eta)^{\frac{4}{n-2}} g_{1}$ denote the solution of the projected problem (3.1). We suppress the dependence on $k$ in $g_{1}$ and $\eta$ but maintain it in the metric $g_{k}$ to avoid confusion with the background metric $g$ on $M$. To indicate the dependence of $g_{k}$ on the conformal structure $\sigma \in \mathcal{S}_{\delta_{3}}\left(\sigma_{0}\right)$ (which determines the approximate solution $\left.g_{1}=g_{1_{k}}(\sigma)\right)$ we write $\left(\sigma, g_{k}\right) \in \mathcal{M}$. We need to show that there exists an admissible conformal structure $\sigma$ whose parameters $\Pi\left(\sigma, g_{k}\right)=\left(b\left(\sigma, g_{k}\right), \mu\left(\sigma, g_{k}\right)\right)$ obtained from $\sigma$ with the solution to the corresponding projected problem $g_{k}$, are the null parameters $(0,0) \in \mathcal{X}$. This is equivalent to the requirement that $g_{k}$ satisfy the hypothesis of Proposition 4.3. Proposition 5.3 shows that the parameters $\Pi\left(\sigma, g_{1}\right)=\left(b\left(\sigma, g_{1}\right), \mu\left(\sigma, g_{1}\right)\right)$ obtained from the deformed conformal structure with its approximate solution metric are close to the parameters which prescribe the deformation. We need to relate these parameters $\left(b\left(\sigma, g_{1}\right), \mu\left(\sigma, g_{1}\right)\right)$ with the parameters obtained from $\sigma$ endowed with the projected solution metric $g_{k},\left(b\left(\sigma, g_{k}\right), \mu\left(\sigma, g_{k}\right)\right)$.

This is done in [15, Proposition 4.2]. Since the parameters are obtained 
by evaluating certain integrals over the boundaries of each asr, comparing the parameters of a fixed conformal structure (which determines the asr's) with two different compatible metrics is purely a local problem in each of the neck regions $N_{ \pm i}^{(\delta)}$. Schoen does this by carefully examining the structure of the metric $g_{k}$ on the neck regions and using the estimates he obtains to compare the Lie algebra elements $X_{e}\left(\sigma, g_{1}\right)$ and $X_{e}\left(\sigma, g_{k}\right)$, where $e= \pm i$ is any edge. We write $X_{e}=X_{e}^{0}+C_{e}$ for the decomposition into rotational and pure dilational parts. Schoen's result is the following

Proposition 6.1. There is a constant $\tau_{4}>0$ such that at each vertex $i, 1 \leq$ $i \leq k$, and each edge $e=+i,-i$, the following inequalities hold:

$$
\begin{aligned}
\left\|X_{e}^{0}\left(\sigma, g_{k}\right)\right\| & \leq \epsilon^{n-2+\tau_{4}}, \\
\left|C_{e}\left(\sigma, g_{k}\right)-C_{e}\left(\sigma, g_{1}\right)\right| & \leq \epsilon^{n-2+\tau_{4}} .
\end{aligned}
$$

Remark 6.1. Although it might appear unnecessary, we have retained Schoen's notation for consistancy with [15] and in order that the constant $\tau_{4}$ is not confused with the parameter $\tau$ used in §5.5. We refer to [15, pages 381-385] for the proof of Proposition 6.1.

The other result we need in order to prove our main Theorem is [15, Proposition 4.5], which asserts that the rotational parts of the Lie algebra elements $X_{i}\left(\sigma, g_{k}\right)$ derived from $\left(\sigma, g_{k}\right)$, are controlled in terms of their pure dilational parts. Let $\mathbf{X}, \mathbf{X}^{0}, \mathbf{C}$ denote the $k$-tuples of the Lie algebra elements $X_{i}$, the rotational parts $X_{i}^{0}$, and the pure dilation parts $C_{i}$ respectively. We define norms for the latter two quantities by

$$
\left\|\mathrm{X}^{0}\right\|=\max _{i}\left\|X_{i}^{0}\right\| \quad \text { and } \quad\|\mathrm{C}\|=\max _{i}\left|C_{i}\right|,
$$

where $\left\|X_{i}^{0}\right\|^{2} \equiv-\frac{1}{2} \operatorname{Tr}\left(\left(X_{i}^{0}\right)^{2}\right)$. The following Proposition is then equivalent to [15, Proposition 4.5].

Proposition 6.2. Suppose that $g_{k}$ is the metric constructed in Theorem 3.1 with respect to the admissible conformal structure $\sigma$. There is a small constant $\epsilon_{10}$ such that

$$
\left\|\mathbf{X}^{0}\left(\sigma, g_{k}\right)\right\| \leq \epsilon_{10}\left\|\mathbf{C}\left(\sigma, g_{k}\right)\right\| .
$$


We refer to [15, pages 388-390] for the proof of Proposition 6.2.

These two Propositions now enable us to prove the existence of the required deformations.

\subsection{Achieving an Exact Solution.}

Theorem 6.3. Given a balanced admissible conformal structure $\sigma_{0}$ on $\mathcal{T}_{k}$, there exists an $\sigma \in \mathcal{D}\left(\mathcal{X}_{\delta}(0,0)\right) \subset \mathcal{S}_{\delta_{3}}\left(\sigma_{0}\right)$, where $\delta=\left(\delta_{1}, \delta_{2}\right)$ and $\delta_{1}, \delta_{2}, \delta_{3}$ are small positive numbers, such that for the metric $g_{k}$ constructed in Theorem 3.1 with respect to $\sigma, R\left(g_{k}\right) \equiv n(n-1)$.

Remark 6.2. Theorem 6.3 is almost identical to [15, Theorem 4.6]. Since it is central to our construction we include its proof here.

Proof. We define a map of the parameter space into itself by evaluating the deformed conformal structure with the metric $g_{k}$ found by solving the associated projected problem. Explicitly, define

$$
\mathbf{T}: \mathcal{X}_{\delta}(0,0) \rightarrow \mathcal{X}
$$

by

$$
\mathbf{T}(b, \mu)=\Pi\left(\mathcal{D}(b, \mu), g_{k}\right)
$$

We write $\sigma=\mathcal{D}(b, \mu)$, so that

$$
\mathbf{T}(b, \mu)=\Pi(\sigma, g)=\left(b\left(\sigma, g_{k}\right), \mu\left(\sigma, g_{k}\right)\right) \in \mathcal{X}
$$

We want to show that these parameters are close to the parameters, $(b, \mu)$ which specify the deformation. We first estimate the difference $\left|b_{i}\left(\sigma, g_{k}\right)-b_{i}\right|$ for each vertex $i$. Since Proposition 5.3 shows that $\left(b\left(\sigma, g_{1}\right), \mu\left(\sigma, g_{1}\right)\right)$ are close to $(b, \mu)$, we have

$$
\begin{aligned}
\left|b_{i}\left(\sigma, g_{k}\right)-b_{i}\right| & \leq\left|b_{i}\left(\sigma, g_{k}\right)-b_{i}\left(\sigma, g_{1}\right)\right|+\left|b_{i}\left(\sigma, g_{1}\right)-b_{i}\right| \\
& \leq\left|b_{i}\left(\sigma, g_{k}\right)-b_{i}\left(\sigma, g_{1}\right)\right|+c\left(\epsilon^{2} \delta_{1}^{1 / 2}+\delta_{2}\right)
\end{aligned}
$$


We use Proposition 6.1 to estimate the first term, as follows

$$
\begin{aligned}
\left|b_{i}\left(\sigma, g_{k}\right)-b_{i}\left(\sigma, g_{1}\right)\right| & =\epsilon^{n-2}\left|\mathbf{P}_{i}\left(C\left(\sigma, g_{k}\right)_{i}-C\left(\sigma, g_{1}\right)_{i}\right)\right| \\
& \leq \epsilon^{n-2}\left(\left|C\left(\sigma, g_{k}\right)_{-i}-C\left(\sigma, g_{1}\right)_{-i}\right|+\left|C\left(\sigma, g_{k}\right)_{+i} C\left(\sigma, g_{1}\right)_{+i}\right|\right) \\
& \leq 2 \epsilon^{\tau_{4}} .
\end{aligned}
$$

Therefore we have

$$
\left|b_{i}\left(\sigma, g_{k}\right)-b_{i}\right| \leq c\left(\epsilon^{2} \delta_{1}^{1 / 2}+\delta_{2}+\epsilon^{\tau_{4}}\right) .
$$

We use Proposition 6.1 again to estimate the difference $\left|\mu_{e}\left(\sigma, g_{k}\right)-\mu_{e}\right|$, for each edge $e=-i,+i$. Notice that from the definition of the parameters $\mu_{e}\left(\sigma, g_{k}\right)$ and $\mu_{e}$, we have

$$
\left|\mu_{e}\left(\sigma, g_{k}\right)-\mu_{e}\right|=\left|\log \left(\frac{\lambda_{e}\left(\sigma, g_{k}\right)}{\lambda_{e}\left(\sigma, g_{1}\right)}\right)\right|
$$

Thus since

$$
\lambda_{e}\left(\sigma, g_{k}\right)=\left[-\left\|X^{0}\left(\sigma, g_{k}\right)_{e}\right\|^{2}+\left|C\left(\sigma, g_{k}\right)_{e}\right|^{2}\right]^{\frac{1}{2-n}}
$$

and

$$
\lambda_{e}\left(\sigma, g_{1}\right)=\left|C\left(\sigma, g_{1}\right)_{e}\right|^{\frac{2}{2-n}}
$$

we find that

$$
\begin{aligned}
\left|\mu_{e}\left(\sigma, g_{k}\right)-\mu_{e}\right| & =\left|\log \left(\frac{-\left\|X^{0}\left(\sigma, g_{k}\right)_{e}\right\|^{2}+\left|C\left(\sigma, g_{k}\right)_{e}\right|^{2}}{\left|C\left(\sigma, g_{1}\right)_{e}\right|^{2}}\right)^{\frac{1}{2-n}}\right| \\
& \leq \frac{2}{n-2}\left|\log \left(\frac{\left|C\left(\sigma, g_{k}\right)_{e}\right|}{C\left(\sigma, g_{1}\right)_{e}}+\frac{\left\|X^{0}\left(\sigma, g_{k}\right)_{e}\right\|}{\left|C\left(\sigma, g_{1}\right)_{e}\right|}\right)\right| .
\end{aligned}
$$

By applying Proposition 6.1 we can estimate

$$
\left|\frac{C\left(\sigma, g_{k}\right)_{e}}{C\left(\sigma, g_{1}\right)_{e}}\right| \leq 1+\frac{\epsilon^{n-2+\tau_{4}}}{\left|C\left(\sigma, g_{1}\right)_{e}\right|}
$$

and

$$
\frac{\left\|X^{0}\left(\sigma, g_{k}\right)_{e}\right\|}{\left|C\left(\sigma, g_{1}\right)_{e}\right|} \leq \frac{\epsilon^{n-2+\tau}}{\left|C\left(\sigma, g_{1}\right)_{e}\right|}
$$

which gives us

$$
\left|\mu_{e}\left(\sigma, g_{k}\right)-\mu_{e}\right| \leq \frac{2}{n-2}\left|\log \left(1+\frac{2 \epsilon^{n 2+\tau_{4}}}{\left|C\left(\sigma, g_{1}\right)_{e}\right|}\right)\right|
$$


Proposition 4.2 explicitly calculates $\left|C\left(\sigma, g_{k}\right)_{e}\right|=\epsilon_{\sigma e}{ }^{n-2}$, thus since $\sigma$ is an admissible conformal structure we have

$$
\begin{aligned}
\left|\mu_{e}\left(\sigma, g_{k}\right)-\mu_{e}\right| & \leq \frac{2}{n-2}\left|\log \left(1+2 \beta^{n 2} \epsilon^{\tau_{4}}\right)\right| \\
& \leq c \epsilon^{\tau_{4}} .
\end{aligned}
$$

It is clear from the definitions of $\mathcal{D}$ and $\Pi$ that $\mathbf{T}$ is continuous with respect to the product topology $\mathcal{X}_{\delta}(0,0)$. Moreover if we choose $\delta_{2} \geq c \epsilon^{\tau_{4}}$ and $\delta_{1} \geq$ $c\left(\epsilon^{2} \delta_{1}^{1 / 2}+\delta_{2}+\epsilon^{\tau_{4}}\right)$, then the estimates (6.1) and (6.2) imply that the map I - T takes the compact, contractible set $\mathcal{X}_{\delta}$ into itself. Therefore we may apply the Schauder fixed point theorem (see [10]) to find parameters $(b, \mu) \in \mathcal{X}_{\delta}(0,0)$ such that

$$
(b, \mu)-\mathbf{T}(b, \mu)=(b, \mu),
$$

and hence

$$
\mathbf{T}(b, \mu)=(0,0) .
$$

If we again let $\sigma$ denote $\mathcal{D}(b, \mu)$ and let $g_{k}$ denote the solution of the projected problem with respect to $\sigma$, then we have

$$
\Pi\left(\sigma, g_{k}\right)=\left(b\left(\sigma, g_{k}\right), \mu\left(\sigma, g_{k}\right)\right)=(0,0) .
$$

We now show that this implies that $R\left(g_{k}\right) \equiv n(n-1)$. Since $\mu\left(\sigma, g_{k}\right)=0$, for each edge $e$, we have

$$
\lambda_{e}\left(\sigma, g_{k}\right)=\lambda_{e}\left(\sigma_{0}, g_{1}\right),
$$

thus since $\sigma_{0}$ is balanced we conclude that for each $i, 1 \leq i \leq k$,

$$
\lambda_{-i}\left(\sigma, g_{k}\right)=\lambda_{+i}\left(\sigma, g_{k}\right) .
$$

Therefore by the definitions of the parameters $\lambda_{e}\left(\sigma, g_{k}\right)$ in terms of the Killing form $B(\cdot, \cdot)$, we have

$$
\left|C_{-i}\left(\sigma, g_{k}\right)\right|-\left\|X_{-i}^{0}\left(\sigma, g_{k}\right)\right\|=\left|C_{+i}\left(\sigma, g_{k}\right)\right|-\left\|X_{+i}^{0}\left(\sigma, g_{k}\right)\right\| .
$$

This can be rewritten as,

$$
\left|\left(C_{-i}+C_{+i}\right) \cdot\left(C_{-i}-C_{+i}\right)\right|=B\left(X_{-i}^{0}+X_{+i}^{0}, X_{-i}^{0} X_{+i}^{0}\right),
$$


where each of the elements of $o(n+1,1)$ corresponds to the pair $\left(\sigma, g_{k}\right) \in \mathcal{M}$. We write

$$
\begin{aligned}
C\left(\sigma, g_{k}\right)_{-i}-C\left(\sigma, g_{k}\right)_{+i}= & C\left(\sigma, g_{1}\right)_{-i}-C\left(\sigma, g_{1}\right)_{+i} \\
& +\left(C\left(\sigma, g_{k}\right)_{-i}-C\left(\sigma, g_{1}\right)_{-i}\right)+\left(C\left(\sigma, g_{k}\right)_{+i} C\left(\sigma, g_{1}\right)_{+i}\right),
\end{aligned}
$$

and use Propositions 4.2 and 6.1 to derive

$$
C\left(\sigma, g_{k}\right)_{-i}-C\left(\sigma, g_{k}\right)_{+i}=\epsilon_{\sigma-i}^{n-2} q_{\sigma-i} \epsilon_{\sigma+i}^{n-2} q_{\sigma+i}+O\left(\epsilon^{n-2+\tau_{4}}\right),
$$

which implies that

$\left|\left(C_{-i}+C_{+i}\right) \cdot\left(q_{\sigma_{-i}}-q_{\sigma_{+} i}\right)\right|=\left\|X_{-i}^{0}+X_{+i}^{0}\right\|\left\|X_{-i}^{0}-X_{+i}^{0}\right\|+c \epsilon^{n 2+\tau_{4}}\left|C_{-i}+C_{+i}\right|$.

Therefore, since $\sigma$ is an admissible conformal structure, for each vertex $i$ we have

$$
\left|C\left(\sigma, g_{k}\right)_{i} \cdot\left(q_{\sigma_{-i}}-q_{\sigma+i}\right)\right| \leq c \epsilon^{n-2}\left\|X_{i}^{0}\right\|\left\|X_{-i}^{0}-X_{+i}^{0}\right\|+c \epsilon^{\tau_{4}}\left|C_{i}\right| .
$$

Thus, by again applying Proposition 6.1 we obtain

$$
\left|C\left(\sigma, g_{k}\right)_{i} \cdot\left(q_{\sigma_{-i}}-q_{\sigma_{+i}}\right)\right| \leq c \epsilon^{\tau_{4}}\left(\left\|X^{0}\left(\sigma, g_{k}\right)_{i}\right\|+\left|C\left(\sigma, g_{k}\right)_{i}\right|\right) .
$$

Since $\sigma \in \mathcal{S}_{\delta_{3}}\left(\sigma_{0}\right)$ we know that for each edge $e,\left|q_{\sigma_{e}}-q_{e}\right|<\delta_{3}$, therefore

$$
\left|C\left(\sigma, g_{k}\right)_{i} \cdot q_{+i}\right| \leq c \epsilon^{\tau_{4}}\left(\left\|X^{0}\left(\sigma, g_{k}\right)_{i}\right\|+\left|C\left(\sigma, g_{k}\right)_{i}\right|\right)+\delta_{3}\left|C\left(\sigma, g_{k}\right)_{i}\right| .
$$

We now use the fact that $b_{i}\left(\sigma, g_{k}\right)=\epsilon^{2-n} \mathbf{P}_{i}\left(C\left(\sigma, g_{k}\right)_{i}\right)=0$, or equivalently

$$
\left|C\left(\sigma, g_{k}\right)_{i}\right|=\left|C\left(\sigma, g_{k}\right)_{i} \cdot q_{+i}\right| \text {. }
$$

Thus we have the estimate

$$
\left|C\left(\sigma, g_{k}\right)_{i}\right| \leq c \epsilon^{\tau_{4}}\left(\left\|X^{0}\left(\sigma, g_{k}\right)_{i}\right\|+\left|C\left(\sigma, g_{k}\right)_{i}\right|\right)+\delta_{3}\left|C\left(\sigma, g_{k}\right)_{i}\right|,
$$

and by choosing $\epsilon$ sufficiently small we conclude that

$$
\left|C\left(\sigma, g_{k}\right)_{i}\right| \leq c \epsilon^{\tau_{4}}\left\|X^{0}\left(\sigma, g_{k}\right)_{i}\right\| .
$$

Therefore by taking the maximum over all vertices $i, 1 \leq i \leq k$ we have

$$
\|\mathbf{C}\| \leq c \epsilon^{\tau_{4}}\left\|\mathbf{X}^{0}\right\| .
$$

By Proposition 6.2 this implies that

$$
\mathbf{C} \equiv 0 \quad \text { and } \quad \mathbf{X}^{0} \equiv 0
$$


which implies that for each vertex $i$,

$$
X\left(\sigma, g_{k}\right)_{i}=0 .
$$

Thus $\left(\sigma, g_{k}\right)$ satisfy the hypothesis of Proposition 4.3 , which we apply to conclude that

$$
R\left(g_{k}\right) \equiv n(n-1)
$$

This completes the proof of Theorem 6.3.

6.3. The Degree of the Metric Perturbation. To complete our proof of Theorem 0.1 it remains to show that the initial metric $\bar{g}$ on $M$ with positive scalar curvature $R(\bar{g})>0$, belongs to a conformal class which is arbitrarily close in $C^{0}$ to the conformal class of any of our $N$ solutions, $g_{k}$. The initial perturbation of $\bar{g}$ which we performed in $\S 1.1$, was to find a new metric $g$, which agrees with $\bar{g}$ outside of a small neighborhood of some point $x_{0}$ and which is locally conformally flat in a slightly smaller neighborhood. This perturbation produces such metrics which are arbitrarily close in $C^{1}$ to $\bar{g}$, and converge to $\bar{g}$ in $C^{1}$ as the size of the neighborhood decreases. The more delicate perturbation is that of the metric on the spherical end $S_{0}^{n}$. While we may find nondegenerate (in the sense of $\$ 1.1$ ) metrics $\bar{g}_{0}$, on $S^{n}$, which are arbitrarily close to $g_{0}$ in as strong a topology as we would like, e.g. $C^{k, \alpha}$, the effect of such a perturbation will not generally result in the conformal class of the corresponding approximate solution $\left[g_{1}\right]$ being arbitrarily close to $[g]$ in $C^{k, \alpha}$. This is because the nondegenerate metric $\bar{g}_{0}$, on $S^{n}$, occurs in $\left(M, g_{1}\right)$ as the pullback $G_{\lambda}^{*}\left(\bar{g}_{0}\right)$, of $\bar{g}_{0}$ under an arbitrarily strong dilation $G_{\lambda}$. Since $\bar{g}_{0}$ is not in the conformal class of $g_{0}, G_{\lambda}^{*}\left(\bar{g}_{0}\right)$ will not, in general, be in the conformal class of $\bar{g}_{0}$.

We now show that the metric $g_{1}$ may be constructed so that it is arbitrarily close in $C^{0}$ to a metric $g^{\prime} \in[g]$. Here $g$ is our nondegenerate minimal solution metric on $M, R(g)=n(n-1)$ and we let $g_{1}$ denote the approximate solution metric which is constructed as in $\S 1.5$ from the balanced conformal structure $\sigma_{0}$ on $\mathcal{T}_{N}$ by using a particular perturbation $\bar{g}_{0}$, of the round metric $g_{0}$ on $S^{n}$. Note that by $\S 1.6$ and $\S 5.5$ each of our $N$ solutions $g_{k}$ where $k=1, \ldots, N$, are in the conformal class of $g_{1}$. 
Proposition 6.4. Given any $\bar{\epsilon}>0$, let $\bar{g}_{0}$ be a metric on $S^{n}$ which is locally conformally flat in the neighborhood of a point, which belongs to a nondegenerate conformal class, and which satisfies

$$
\begin{aligned}
R\left(\bar{g}_{0}\right) & =n(n-1) \\
\left\|g_{0}-\bar{g}_{0}\right\|_{C^{0}} & <\bar{\epsilon}
\end{aligned}
$$

and let $g_{1}$ be the metric constructed from $\sigma_{0}$, the balanced admissible conformal structure on $\mathcal{T}_{N}$, using the metric $\bar{g}_{0}$ as in $\S 1.5$. Then there exists a metric $g^{\prime} \in[g]$ which satisfies

$$
\left\|g_{1}-g^{\prime}\right\|_{C^{0}}<\bar{\epsilon}
$$

Proof. We take $g^{\prime}$ to be the metric constructed in the identical manner as $g_{1}$ via $\sigma_{0}$, except that we use the standard round metric $g_{0}$ on $S^{n}$ rather than the metric $\bar{g}_{0}$ on the spherical end $S_{0}^{n}$. The fact that $g^{\prime} \in[g]$ is immediate from the construction of the approximate solutions, $g^{\prime}$ differs from $g$ only in a small neighborhood $B_{r}\left(x_{0}\right) \subset M$, where $g$ is conformally flat. The metrics $g_{1}$ and $g^{\prime}$ are isometric in $M \backslash S_{0}^{n}$. The result does not follow immediately from the assumption on the metrics $\bar{g}_{0}$ and $g_{0}$ since $S_{0}^{n}$ and $M_{0}$ are separated by an annulus of large conformal modulus. Thus the effect of the construction of $g_{1}$ and $g^{\prime}$ on these metrics is conformally equivalent to taking the pullback of the metrics $g_{0}$ and $\bar{g}_{0}$ under a strong centered dilation $G_{\lambda}$. The strength $\lambda$ of this dilation depends on $N$, the number of solutions we construct and on the number $\epsilon$ used in the definition of an admissible conformal structure (1.6). In particular $\lambda$ tends to infinity as either $N$ tends to infinity or as $\epsilon$ tends to zero. Consider the metrics $g_{\lambda}$ and $\bar{g}_{\lambda}$ on $S^{n}$ defined by

$$
g_{\lambda}=G_{\lambda}^{*}\left(g_{0}\right) \quad \text { and } \quad \bar{g}_{\lambda}=G_{\lambda}^{*}\left(\bar{g}_{0}\right)
$$

and let $h$ and $h_{\lambda}$ be the symmetric $(0,2)$ tensors on $S^{n}$ defined by

$$
h=g_{0}-\bar{g}_{0} \quad \text { and } \quad h_{\lambda}=g_{\lambda}-\bar{g}_{\lambda} .
$$

Notice that $G_{\lambda}$ is a conformal diffeomorphism of $\left(S^{n}, g_{0}\right)$,

$$
g_{\lambda}=\left|G_{\lambda}^{\prime}\right|^{2} g_{0},
$$


where $\left|G_{\lambda}^{\prime}\right|$ is the linear stretch factor of the dilation $G_{\lambda}$. This will not be the case for $\bar{g}_{\lambda}=G_{\lambda}^{*}\left(\bar{g}_{0}\right)$. We may compute the $C^{0}$ norms of $h$ and $h_{\lambda}$ with respect to a fixed metric, for example $g_{0}$, by writing these tensors in stereographic coordinates on $S^{n} \backslash\{q\}$, where $q$ is the source of $G_{\lambda}$. This computation easily shows that

$$
\left\|h_{\lambda}(x)\right\|=\|h(\lambda x)\|
$$

therefore

$$
\left\|h_{\lambda}\right\|_{C^{0}}=\|h\|_{C^{0}} .
$$

Thus since $h=g_{0}-\bar{g}_{0}$, we have $\|h\|_{C^{0}}<\bar{\epsilon}$, the desired estimate follows from (6.3). This completes the proof of Proposition 6.4.

\section{REFERENCES}

1. Aubin, T., The scalar curvature, Differential Geometry and Relativity (Cahen and Flato, eds), Reider, 1976.

2. Chavel, I., Eigenvalues in Riemannian Geometry, Academic Press, 1984.

3. Gidas, B., Ni, W.M. and Nirenberg, L., Symmetry and related properties via the maximum principle, Comm. Math. Phys. 68 (1979) 209-243.

4. Gilbarg, D. and Trudinger, N., Elliptic Partial Differential Equations of Second Order, Springer-Verlag, Berlin, second edition, 1983.

5. Kapouleas, N., Compact constant mean curvature surfaces in Euclidean threespace, J. Differential Geom. 33 (1991) 683-715.

6. Kapouleas, N., Complete constant mean curvature surfaces in Euclidean threespace, Ann. of Math. (2) 181 (1990) 239-330.

7. Kazdan, J., and Warner, F., Curvature functions for compact 2-manifolds, Ann. of Math. (2) 99 (1974) 14-74.

8. Kobayashi, O., Scalar curvature of a metric with unit volume, Math. Annal.279 (1987) 253- 265.

9. Lee, J.M. and Parker, T.H., The Yamabe problem, Bull. Amer. Math. Soc. (N.S.) 17 (1987) 37-91.

10. Nirenberg, L., Topics in Nonlinear Functional Analysis, NYU Lecture Notes, 1973.

11. Obata, M., The conjectures on conformal transformations of Riemannian manifolds, J. Differential Geom. 6 (1972) 247-258.

12. Pohozaev, S., Eigenfunctions of the equation $\Delta u+\lambda f(u)=0$, Soviet Math. Doklady, 6 (1965) 1408-1411.

13. Rellich, R., Über das asymptotische Verhalten der Lösugen von $\Delta u+\lambda u=0$ in unendlichen Gebieten, Jahresber. Deutsch. Math. verein 53 (1943), 57-65. 
14. Schoen, R., Conformal deformation of a Riemannian metric to constant scalar curvature, J. Differential Geometry 20 (1984) 479-495.

15. Schoen, R., The existence of weak solutions with prescribed singular behavior for a conformally invariant scalar equation, Comm. Pure Appl. Math. 41 (1988) 317-392.

16. Schoen, R., On the number of constant scalar curvature metrics in a conformal class, preprint.

17. Schoen, R., A report on some recent progress on nonlinear problems in geometry, Surveys in Differential Geometry, Supplement to J. Differential Geom. 1 (1991) 201-241.

18. Schoen, R., Variational theory for the total scalar curvature functional for Riemannian metrics and related topics, Topics in Calculus of Variations (M. Giaquinta, ed.), Lecture Notes in Math., Vol. 1365, Springer-Verlag, Berlin, 1987.

19. Trudinger, N., Remarks concerning the conformal deformation of Riemannian structures on compact manifolds, Ann. Scuola Norm. Sup. Pisa Cl. Sci. (4) 22 (1968) 265-274.

20. Yamabe, H., On a deformation of Riemannian structures on compact manifolds, Osaka J. Math. 12 (1960) 21-37.

The University of Texas at Austin, U. S. A.

RECEIVED OCTOBER 26, 1992 REvised JANUARy 25, 1993 